\title{
Critical edge behavior in unitary random matrix ensembles and the thirty fourth Painlevé transcendent
}

\author{
A.R. Its \\ Department of Mathematical Sciences \\ Indiana University - Purdue University Indianapolis \\ Indianapolis IN 46202-3216, U.S.A. \\ itsa@math.iupui.edu \\ A.B.J. Kuijlaars and J. Östensson \\ Department of Mathematics \\ Katholieke Universiteit Leuven \\ Celestijnenlaan 200B \\ 3001 Leuven, Belgium \\ arno.kuijlaars@wis.kuleuven.be \\ ostensson@wis.kuleuven.be
}

June 3, 2018

\begin{abstract}
We describe a new universality class for unitary invariant random matrix ensembles. It arises in the double scaling limit of ensembles of random $n \times n$ Hermitian matrices $Z_{n, N}^{-1}|\operatorname{det} M|^{2 \alpha} e^{-N \operatorname{Tr} V(M)} d M$ with $\alpha>-1 / 2$, where the factor $|\operatorname{det} M|^{2 \alpha}$ induces critical eigenvalue behavior near the origin. Under the assumption that the limiting mean eigenvalue density associated with $V$ is regular, and that the origin is a right endpoint of its support, we compute the limiting eigenvalue correlation kernel in the double scaling limit as $n, N \rightarrow \infty$ such that $n^{2 / 3}(n / N-1)=O(1)$. We use the Deift-Zhou steepest descent method for the Riemann-Hilbert problem for polynomials on the line orthogonal with respect to the weight $|x|^{2 \alpha} e^{-N V(x)}$. Our main attention is on the construction of a local parametrix near the origin by means of the $\psi$-functions associated with a distinguished solution of the Painlevé XXXIV equation. This solution is related to a particular solution of the Painlevé II equation, which however is different from the usual Hastings-McLeod solution.
\end{abstract}

2000 Mathematics Subject Classification: 15A52, 33E17, 34M55 


\section{Introduction and statement of results}

\subsection{Unitary random matrix models}

For $n \in \mathbb{N}, N>0$, and $\alpha>-1 / 2$, we consider the unitary random matrix ensemble

$$
Z_{n, N}^{-1}|\operatorname{det} M|^{2 \alpha} e^{-N \operatorname{Tr} V(M)} d M
$$

on the space $\mathcal{M}(n)$ of $n \times n$ Hermitian matrices $M$, where $V$ is real analytic and satisfies

$$
\lim _{x \rightarrow \pm \infty} \frac{V(x)}{\log \left(x^{2}+1\right)}=+\infty
$$

This is a unitary random matrix ensemble in the sense that it is invariant under conjugation, $M \mapsto U M U^{-1}$, by unitary matrices $U$. As is well-known [11, 38], it induces the following probability density on the $n$ eigenvalues $x_{1}, \ldots, x_{n}$ of $M$

$$
P^{(n, N)}\left(x_{1}, \ldots, x_{n}\right)=\widehat{Z}_{n, N}^{-1} \prod_{j=1}^{n}\left|x_{j}\right|^{2 \alpha} e^{-N V\left(x_{j}\right)} \prod_{i<j}\left|x_{i}-x_{j}\right|^{2} .
$$

The eigenvalue distribution is determinantal with kernel $K_{n, N}$ built out of the polynomials $p_{j, N}(x)=\kappa_{j, N} x^{j}+\cdots, \kappa_{j, N}>0$, orthonormal with respect to the weight $|x|^{2 \alpha} e^{-N V(x)}$ on $\mathbb{R}$. Indeed, as shown by Dyson, Gaudin, and Mehta, see e.g. [11, 21, 38], for any $m=1, \ldots, n-1$, the $m$-point correlation function

$$
R_{m}^{(n, N)}\left(x_{1}, \ldots, x_{m}\right) \equiv \frac{n !}{(n-m) !} \int_{-\infty}^{\infty} \cdots \int_{-\infty}^{\infty} P^{(n, N)}\left(x_{1}, \ldots, x_{n}\right) d x_{m+1} \cdots d x_{n}
$$

is given by

$$
R_{m}^{(n, N)}\left(x_{1}, \ldots, x_{m}\right)=\operatorname{det}\left(K_{n, N}\left(x_{i}, x_{j}\right)\right)_{1 \leq i, j \leq m}
$$

where

$$
K_{n, N}(x, y)=|x|^{\alpha}|y|^{\alpha} e^{-\frac{1}{2} N(V(x)+V(y))} \sum_{j=0}^{n-1} p_{j, N}(x) p_{j, N}(y) .
$$

In the limit $n, N \rightarrow \infty, n / N \rightarrow 1$, the global eigenvalue regime is determined by $V$ as follows. The equilibrium measure $\mu_{V}$ for $V$ is the unique minimizer of

$$
I_{V}(\mu)=\iint \log \frac{1}{|x-y|} d \mu(x) d \mu(y)+\int V(x) d \mu(x)
$$

taken over all Borel probability measures $\mu$ on $\mathbb{R}$. Since $V$ is real analytic we have that $\mu_{V}$ is supported on a finite union of disjoint intervals [13], and it has a density $\rho_{V}$ such that

$$
\lim _{n, N \rightarrow \infty, n / N \rightarrow 1} \frac{1}{n} K_{n, N}(x, x)=\rho_{V}(x), \quad x \neq 0 .
$$

The limiting mean eigenvalue density is independent of $\alpha$. 
The factor $|\operatorname{det} M|^{2 \alpha}$ changes the local eigenvalue behavior near 0 . This is reflected in the local scaling limits of $K_{n, N}$ around 0 that do depend on $\alpha$. If 0 is in the bulk of the spectrum and $\rho_{V}(0)>0$, then instead of the usual sine kernel we get a Bessel kernel depending on $\alpha$ [37]. If 0 is in the bulk and $\rho_{V}(0)=\rho_{V}^{\prime}(0)=0, \rho_{V}^{\prime \prime}(0)>0$, then the local scaling limits of the kernel near 0 are associated with the Hastings-McLeod solution of the Painlevé II equation $q^{\prime \prime}=s q+2 q^{3}-\alpha[9]$.

In this paper we study the effect of $\alpha$ in case 0 is an endpoint of the spectrum which is such that the density $\rho_{V}$ vanishes like a square root at 0 . For $\alpha=0$ the scaling limit is the well-known Airy kernel, see the papers [6, 26, 39, 42] and also [3, 12], and so we are asking the question: What is the $\alpha$-generalization of the Airy kernel?

For $\alpha>-1 / 2$, we have found a new one-parameter family of limiting kernels as stated in Theorem 1.1 below. In Theorem 1.1 we also assume that the eigenvalue density $\rho_{V}$ is regular, which means the following.

- The function $x \mapsto 2 \int \log |x-s| \rho_{V}(s) d s-V(x)$ defined for $x \in \mathbb{R}$, assumes its maximum value only on the support of $\rho_{V}$.

- The density $\rho_{V}$ is positive on the interior of its support.

- The density $\rho_{V}$ vanishes like a square root at each of the endpoints of its support.

Theorem 1.1 For every $\alpha>-1 / 2$, there exists a one-parameter family of kernels $K_{\alpha}^{\text {edge }}(x, y ; s)$ such that the following holds. Let $V$ be a real analytic external field on $\mathbb{R}$ such that its mean limiting eigenvalue density $\rho_{V}$ is regular. Suppose that 0 is a right endpoint of the support of $\rho_{V}$ so that for some constant $c_{1}=c_{1, V}>0$

$$
\rho_{V}(x) \sim \frac{c_{1}}{\pi}|x|^{1 / 2} \quad \text { as } x \rightarrow 0-.
$$

Then there exists a second constant $c_{2}=c_{2, V}>0$ such that

$$
\lim _{n, N \rightarrow \infty} \frac{1}{\left(c_{1} n\right)^{2 / 3}} K_{n, N}\left(\frac{x}{\left(c_{1} n\right)^{2 / 3}}, \frac{y}{\left(c_{1} n\right)^{2 / 3}}\right)=K_{\alpha}^{\text {edge }}(x, y ; s)
$$

whenever $n, N \rightarrow \infty$ such that

$$
\lim _{n \rightarrow \infty} n^{2 / 3}\left(\frac{n}{N}-1\right)=L \in \mathbb{R}
$$

and $s=-c_{2, V} L$.

For $\alpha=0$, the limiting kernels reduce to the kernel

$$
K_{0}^{e d g e}(x, y ; s)=\frac{\operatorname{Ai}(x+s) \operatorname{Ai}^{\prime}(y+s)-\operatorname{Ai}^{\prime}(x+s) \operatorname{Ai}(y+s)}{x-y},
$$

which is the (shifted) Airy kernel from random matrix theory mentioned above, see also Subsection 4.1 below. For $\alpha \neq 0$, a new type of special functions is needed to describe the limiting kernel $K_{\alpha}^{e d g e}(x, y ; s)$. This description is given in the next subsections. 


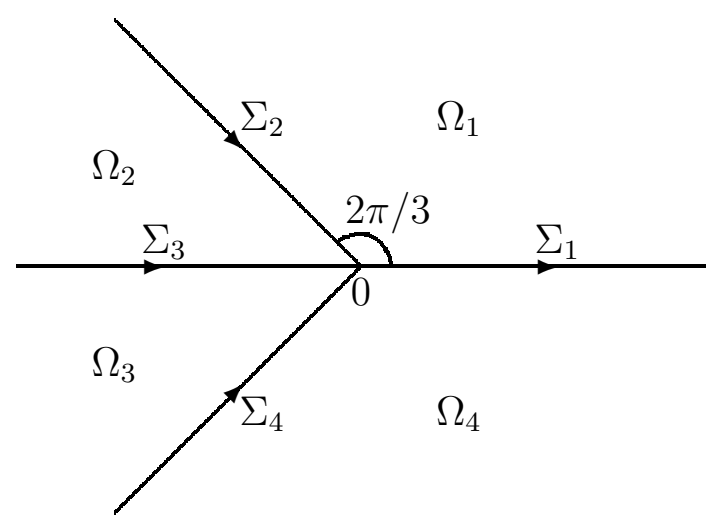

Figure 1: Contour for the model RH problem.

\subsection{The model RH problem}

We describe $K_{\alpha}^{e d g e}(x, y ; s)$ through the solution of a special Riemann-Hilbert $(\mathrm{RH})$ problem, that we will refer to as the model RH problem.

The model RH problem is posed on a contour $\Sigma$ in an auxiliary $\zeta$-plane, consisting of four rays $\Sigma_{1}=\{\arg \zeta=0\}, \Sigma_{2}=\{\arg \zeta=2 \pi / 3\}, \Sigma_{3}=\{\arg \zeta=\pi\}$, and $\Sigma_{4}=\{\arg \zeta=$ $-2 \pi / 3\}$ with orientation as shown in Figure 1. As usual in RH problems, the orientation defines $\mathrm{a}+$ and $\mathrm{a}-$ side on each part of the contour, where the +-side is on the left when traversing the contour according to its orientation. For a function $f$ on $\mathbb{C} \backslash \Sigma$, we use $f_{ \pm}$ to denote its limiting values on $\Sigma$ taken from the \pm -side, provided such limiting values exist. The contour $\Sigma$ divides the complex plane into four sectors $\Omega_{j}$ also shown in the figure.

The model RH problem reads as follows.

\section{Riemann-Hilbert problem for $\Psi_{\alpha}$}

(a) $\Psi_{\alpha}: \mathbb{C} \backslash \Sigma \rightarrow \mathbb{C}^{2 \times 2}$ is analytic.

(b) $\Psi_{\alpha,+}(\zeta)=\Psi_{\alpha,-}(\zeta)\left(\begin{array}{ll}1 & 1 \\ 0 & 1\end{array}\right)$, for $\zeta \in \Sigma_{1}$,

$\Psi_{\alpha,+}(\zeta)=\Psi_{\alpha,-}(\zeta)\left(\begin{array}{cc}1 & 0 \\ e^{2 \alpha \pi i} & 1\end{array}\right)$, for $\zeta \in \Sigma_{2}$,

$\Psi_{\alpha,+}(\zeta)=\Psi_{\alpha,-}(\zeta)\left(\begin{array}{cc}0 & 1 \\ -1 & 0\end{array}\right)$, for $\zeta \in \Sigma_{3}$,

$\Psi_{\alpha,+}(\zeta)=\Psi_{\alpha,-}(\zeta)\left(\begin{array}{cc}1 & 0 \\ e^{-2 \alpha \pi i} & 1\end{array}\right)$, for $\zeta \in \Sigma_{4}$.

(c) $\Psi_{\alpha}(\zeta)=\zeta^{-\sigma_{3} / 4} \frac{1}{\sqrt{2}}\left(\begin{array}{ll}1 & i \\ i & 1\end{array}\right)\left(I+O\left(1 / \zeta^{1 / 2}\right)\right) e^{-\left(\frac{2}{3} \zeta^{3 / 2}+s \zeta^{1 / 2}\right) \sigma_{3}}$ as $\zeta \rightarrow \infty$. Here $\sigma_{3}=$ $\left(\begin{array}{cc}1 & 0 \\ 0 & -1\end{array}\right)$ is the third Pauli matrix.

(d) $\Psi_{\alpha}(\zeta)=O\left(\begin{array}{ll}|\zeta|^{\alpha} & |\zeta|^{\alpha} \\ |\zeta|^{\alpha} & |\zeta|^{\alpha}\end{array}\right)$ as $\zeta \rightarrow 0$, if $\alpha<0$; and 


$$
\Psi_{\alpha}(\zeta)=\left\{\begin{array}{cc}
O\left(\begin{array}{ll}
|\zeta|^{\alpha} & |\zeta|^{-\alpha} \\
|\zeta|^{\alpha} & |\zeta|^{-\alpha}
\end{array}\right) & \text { as } \zeta \rightarrow 0 \text { with } \zeta \in \Omega_{1} \cup \Omega_{4}, \\
O\left(\begin{array}{ll}
|\zeta|^{-\alpha} & |\zeta|^{-\alpha} \\
|\zeta|^{-\alpha} & |\zeta|^{-\alpha}
\end{array}\right) & \text { as } \zeta \rightarrow 0 \text { with } \zeta \in \Omega_{2} \cup \Omega_{3},
\end{array} \quad \text { if } \alpha \geq 0\right.
$$

Here, and in what follows, the $O$-terms are taken entrywise. Note that the RH problem depends on a parameter $s$ through the asymptotic condition at infinity. If we want to emphasize the dependence on $s$ we will write $\Psi_{\alpha}(\zeta ; s)$ instead of $\Psi_{\alpha}(\zeta)$.

The model RH problem is not uniquely solvable. Indeed, if $\Psi_{\alpha}$ is a solution, then $\left(\begin{array}{ll}1 & 0 \\ \eta & 1\end{array}\right) \Psi_{\alpha}$ is also a solution for any $\eta=\eta(s)$, and it turns out that this is the only freedom we have (see Proposition 2.1).

Theorem 1.2 The model $R H$ problem is solvable for every $s \in \mathbb{R}$. Let $\Psi_{\alpha}$ be a solution of the model $R H$ problem and put

$$
\left(\begin{array}{l}
\psi_{1}(x ; s) \\
\psi_{2}(x ; s)
\end{array}\right)= \begin{cases}\Psi_{\alpha,+}(x ; s)\left(\begin{array}{l}
1 \\
0
\end{array}\right), & \text { for } x>0 \\
\Psi_{\alpha,+}(x ; s) e^{-\alpha \pi i \sigma_{3}}\left(\begin{array}{l}
1 \\
1
\end{array}\right), & \text { for } x<0\end{cases}
$$

Then the limiting kernel $K_{\alpha}^{e d g e}(x, y ; s)$ can be written in the "integrable form"

$$
K_{\alpha}^{e d g e}(x, y ; s)=\frac{\psi_{2}(x ; s) \psi_{1}(y ; s)-\psi_{1}(x ; s) \psi_{2}(y ; s)}{2 \pi i(x-y)} .
$$

The function $\psi_{2}$ depends on the particular choice of solution $\Psi_{\alpha}$ to the model RH problem. Indeed, for any $\eta$ we have that the mapping $\Psi_{\alpha} \mapsto\left(\begin{array}{ll}1 & 0 \\ \eta & 1\end{array}\right) \Psi_{\alpha}$ leaves $\psi_{1}$ invariant and changes $\psi_{2}$ to $\psi_{2}+\eta \psi_{1}$. However, this does not change the expression (1.13) for the kernel $K_{\alpha}^{e d g e}(x, y ; s)$.

It follows from (1.12) and part (c) of the model $\mathrm{RH}$ problem that $\psi_{1}$ and $\psi_{2}$ have the asymptotic behavior

$$
\begin{aligned}
& \psi_{1}(x ; s)=\frac{1}{\sqrt{2} x^{1 / 4}} e^{-\frac{2}{3} x^{3 / 2}-s x^{1 / 2}}\left(1+O\left(x^{-1 / 2}\right)\right), \\
& \psi_{2}(x ; s)=\frac{i x^{1 / 4}}{\sqrt{2}} e^{-\frac{2}{3} x^{3 / 2}-s x^{1 / 2}}\left(1+O\left(x^{-1 / 2}\right)\right)
\end{aligned}
$$

as $x \rightarrow+\infty$, and

$$
\begin{aligned}
& \psi_{1}(x ; s)=\sqrt{2}|x|^{-1 / 4} \cos \left(\frac{2}{3}|x|^{3 / 2}-s|x|^{1 / 2}-\alpha \pi-\pi / 4\right)+O\left(x^{-3 / 4}\right), \\
& \psi_{2}(x ; s)=-i \sqrt{2}|x|^{1 / 4} \sin \left(\frac{2}{3}|x|^{3 / 2}-s|x|^{1 / 2}-\alpha \pi-\pi / 4\right)+O\left(x^{-1 / 4}\right),
\end{aligned}
$$

as $x \rightarrow-\infty$.

Remark 1.3 The kernel $K_{\alpha}^{e d g e}(x, y ; s)$ describes an edge effect for the random matrix ensemble (1.1). If we assume that 0 is the rightmost point in the support of $\rho_{V}$, and if given $M$ we let $\lambda_{\max }(M)$ denote its largest eigenvalue, then it follows under the assumptions of Theorem 1.1, in particular the limit assumption (1.10), that

$$
\lim _{n, N \rightarrow \infty} \mathbb{P}\left(\left(c_{1} n\right)^{2 / 3} \lambda_{\max } \leq t\right)=\operatorname{det}\left(1-\left.K_{\alpha, s}\right|_{(t, \infty)}\right)
$$


where $\left.K_{\alpha, s}\right|_{(t, \infty)}$ is the trace class operator in $L^{2}(t, \infty)$ with kernel $K_{\alpha}^{e d g e}(x, y ; s)$. To prove (1.18) ) one must show that the operator with kernel $\frac{1}{\left(c_{1} n\right)^{2 / 3}} K_{n, N}\left(\frac{x}{\left(c_{1} n\right)^{2 / 3}}, \frac{y}{\left(c_{1} n\right)^{2 / 3}}\right)$ converges in the trace class norm on $L^{2}(t, \infty)$ to the operator with kernel $K_{\alpha}^{e d g e}(x, y ; s)$. This requires good estimates on the rate of convergence in (1.9), which can be established as in 12 .

For $\alpha=0$, the kernel is the (shifted) Airy kernel, and the Fredholm determinant (1.18) has an equivalent expression in terms of a special solution of the Painlevé II equation. The resulting distribution is the famous Tracy-Widom distribution [42, 43]. It would be very interesting to find an analogous expression for general $\alpha$. The connection to the model RH problem given in Theorem 1.2 can be used in obtaining such an expression, following the approach of [5] and [27]. We are planning to address this question in a future publication.

\subsection{Connection with the Painlevé XXXIV equation}

The model $\mathrm{RH}$ problem is related to a special solution of the equation number XXXIV from the list of Painlevé and Gambier [29],

$$
u^{\prime \prime}=4 u^{2}+2 s u+\frac{\left(u^{\prime}\right)^{2}-(2 \alpha)^{2}}{2 u} .
$$

All solutions of $(\underline{1.19})$ are meromorphic in the complex plane.

Theorem 1.4 Let $\Psi_{\alpha}(\zeta ; s)$ be a solution of the model $R H$ problem. Then

$$
u(s)=-\frac{s}{2}-i \frac{d}{d s} \lim _{\zeta \rightarrow \infty}\left[\zeta\left(\Psi_{\alpha}(\zeta ; s) e^{\left(\frac{2}{3} \zeta^{3 / 2}+s \zeta^{1 / 2}\right) \sigma_{3}} \frac{1}{\sqrt{2}}\left(\begin{array}{cc}
1 & -i \\
-i & 1
\end{array}\right) \zeta^{\sigma_{3} / 4}\right)_{12}\right]
$$

exists and satisfies (1.19). The function (1.20) is a global solution of (1.19) (i.e., it does not have poles on the real line), and it does not depend on the particular solution of the model $R H$ problem.

The connection with the Painlevé XXXIV equation leads to the following characterization of $\psi_{1}$ and $\psi_{2}$.

Theorem 1.5 Let $u$ be the solution of Painlevé XXXIV given by (1.20). Then there exists a solution $\Psi_{\alpha}$ of the model $R H$ problem so that the functions $\psi_{1}$ and $\psi_{2}$ defined by (1.12) satisfy the following system of linear differential equations

$$
\frac{d}{d x}\left(\begin{array}{c}
\psi_{1}(x ; s) \\
\psi_{2}(x ; s)
\end{array}\right)=\left(\begin{array}{cc}
u^{\prime} /(2 x) & i-i u / x \\
-i\left(x+s+u+\left(\left(u^{\prime}\right)^{2}-(2 \alpha)^{2}\right) /(4 u x)\right) & -u^{\prime} /(2 x)
\end{array}\right)\left(\begin{array}{l}
\psi_{1}(x ; s) \\
\psi_{2}(x ; s)
\end{array}\right)
$$

and have asymptotics (1.14)-(1.17) .

In fact we will prove that for $\zeta \in \mathbb{C} \backslash \Sigma$,

$$
\frac{d}{d \zeta} \Psi_{\alpha}(\zeta ; s)=\left(\begin{array}{cc}
u^{\prime} /(2 \zeta) & i-i u / \zeta \\
-i\left(\zeta+s+u+\left(\left(u^{\prime}\right)^{2}-(2 \alpha)^{2}\right) /(4 u \zeta)\right) & -u^{\prime} /(2 \zeta)
\end{array}\right) \Psi_{\alpha}(\zeta ; s)
$$


from which (1.21) readily follows in view of (1.12). We emphasize that (1.21) and (1.22) hold for one particular solution of the model RH problem. Any other solution $\left(\begin{array}{ll}1 & 0 \\ \eta & 0\end{array}\right) \Psi_{\alpha}(\zeta ; s)$ also satisfies a system of linear differential equations, but with matrix

$$
\left(\begin{array}{ll}
1 & 0 \\
\eta & 1
\end{array}\right)\left(\begin{array}{cc}
u^{\prime} /(2 \zeta) & i-i u / \zeta \\
-i\left(\zeta+s+u+\left(\left(u^{\prime}\right)^{2}-(2 \alpha)^{2}\right) /(4 u \zeta)\right) & -u^{\prime} /(2 \zeta)
\end{array}\right)\left(\begin{array}{cc}
1 & 0 \\
-\eta & 1
\end{array}\right)
$$

In order to make Theorem 1.5 a genuine, i.e., independent of the $\Psi_{\alpha} \mathrm{RH}$ problem, characterization of $\psi_{1}$ and $\psi_{2}$, we need an independent of formula (1.20) characterization of the solution $u(s)$ of equation (1.19). This can be achieved by indicating the asymptotic behavior of $u(s)$ as $s \rightarrow \infty$, cf. the characterization of the Hastings-McLeod solution of Painlevé II equation [28]. We discuss this issue in detail in the last section of the paper, see in particular Proposition 4.1 and the end of Remark 4.2 where the possible asymptotic characterizations of the solution $u(s)$ are given.

\subsection{Overview of the rest of the paper}

In Section 2 we give the proofs of Theorem [1.1 and Theorem 1.2, We start by presenting the RH problem for orthogonal polynomials on the line [23]. The eigenvalue correlation kernel $K_{n, N}$ can be explicitly expressed in terms of the solution of this RH problem [11, 15]. As in earlier papers, see e.g. [3, 4, 8, 9, 14, 15], we apply the Deift-Zhou steepest descent method for RH problems, see [16]. For the local analysis near 0, we need the model RH problem for $\Psi_{\alpha}(\zeta ; s)$ as introduced in Subsection 1.2. We show, following the methodology of [25], that the model RH problem has a solution for every $s \in \mathbb{R}$. Then we follow the usual steps in the steepest descent analysis for RH problems, which lead us to the proofs of Theorem 1.1 and 1.2 .

Section 3 is devoted to the proofs of Theorem 1.4 and Theorem 1.5. We start by discussing the RH problem, in the form due to Flaschka and Newell, associated with the Painlevé II equation $q^{\prime \prime}=s q+2 q^{3}-\nu$. Following [2], we show that for a special choice of monodromy data the Flaschka-Newell RH problem is related to the model RH problem. The parameters in the Painlevé equations are related by $\nu=2 \alpha+1 / 2$. The monodromy data corresponds to a solution of Painlevé II which is different from the Hastings-McLeod solution that has appeared more often in random matrix theory [4, 8, 9, 28, 42]. The known results (asymptotics, Lax pair etc.) for the RH problem for Painlevé II are then transferred to the model RH problem, and then used to complete the proofs of Theorems 1.4 and 1.5. In particular it gives rise to the special solution $u$ of the Painlevé XXXIV equation defined by (1.20).

In Section 4 we make some concluding remarks. For the important special cases $\alpha=0$ and $\alpha=1$, we show how the model RH problem can be explicitly solved in terms of Airy functions, and how the limiting kernel $K_{\alpha}^{e d g e}(x, y ; s)$ as well as the special Painlevé XXXIV solution $u$ can be explicitly computed in both cases. Our final remarks concern the characterization of $u$, in the case of general $\alpha$, through its asymptotic behavior at infinity. 


\section{Proof of Theorem 1.1 and 1.2}

\subsection{The Riemann-Hilbert problem for orthogonal polynomials}

The RH problem for orthogonal polynomials on the line, for our particular weight, is the following (cf. [23]).

\section{Riemann-Hilbert problem for $Y$}

- $Y: \mathbb{C} \backslash \mathbb{R} \rightarrow \mathbb{C}^{2 \times 2}$ is analytic.

- $Y_{+}(x)=Y_{-}(x)\left(\begin{array}{cc}1 & |x|^{2 \alpha} e^{-N V(x)} \\ 0 & 1\end{array}\right)$ for $x \in \mathbb{R} \backslash\{0\}$, with $\mathbb{R}$ oriented from left to right.

- $Y(z)=(I+O(1 / z))\left(\begin{array}{cc}z^{n} & 0 \\ 0 & z^{-n}\end{array}\right)$ as $z \rightarrow \infty$.

- If $\alpha<0$, then $Y(z)=O\left(\begin{array}{l}1|z|^{2 \alpha} \\ 1|z|^{2 \alpha}\end{array}\right)$ as $z \rightarrow 0$. If $\alpha \geq 0$, then $Y(z)=O\left(\begin{array}{ll}1 & 1 \\ 1 & 1\end{array}\right)$ as $z \rightarrow 0$.

The RH problem has the unique solution

$$
Y(z)=\left(\begin{array}{cc}
\frac{1}{\kappa_{n, N}} p_{n, N}(z) & \frac{1}{2 \pi i \kappa_{n, N}} \int_{\mathbb{R}} \frac{p_{n, N}(s)|s|^{2 \alpha} e^{-N V(s)}}{s-z} d s \\
-2 \pi i \kappa_{n-1, N} p_{n-1, N}(z) & -\kappa_{n-1, N} \int_{\mathbb{R}} \frac{p_{n-1, N}(s)|s|^{2 \alpha} e^{-N V(s)}}{s-z} d s
\end{array}\right)
$$

where $p_{j, N}(x)=\kappa_{j, N} x^{j}+\cdots$ is the orthonormal polynomial with respect to the weight $|x|^{2 \alpha} e^{-N V(x)}$. By (1.6) and the Christoffel-Darboux formula for orthogonal polynomials, we have

$$
K_{n, N}(x, y)=|x|^{\alpha}|y|^{\alpha} e^{-\frac{1}{2} N(V(x)+V(y))} \frac{\kappa_{n-1, N}}{\kappa_{n, N}} \frac{p_{n, N}(x) p_{n-1, N}(y)-p_{n-1, N}(x) p_{n, N}(y)}{x-y} .
$$

Thus, using (2.1) and the fact that $\operatorname{det} Y \equiv 1$, we may express the eigenvalue correlation kernel directly in terms of $Y$ :

$$
K_{n, N}(x, y)=\frac{1}{2 \pi i(x-y)}|x|^{\alpha}|y|^{\alpha} e^{-\frac{1}{2} N(V(x)+V(y))}\left(\begin{array}{ll}
0 & 1
\end{array}\right) Y_{+}^{-1}(y) Y_{+}(x)\left(\begin{array}{l}
1 \\
0
\end{array}\right) .
$$

The main idea for the proof of Theorems 1.1 and 1.2 is to apply the powerful steepest descent analysis for RH problems of Deift and Zhou [16] to the RH problem satisfied by $Y$. In the case at hand it consists of constructing a sequence of invertible transformations $Y \mapsto T \mapsto S \mapsto R$, where the matrix-valued function $R$ is close to the identity. By unfolding the above transformations asymptotics for $Y$ and thus, in view of (2.3), for $K_{n, N}$ in various regimes may be derived. Our main attention will be devoted to the local behavior of $Y$ near 0 . Around 0 we construct a local parametrix with the help of the model RH problem, which we next discuss in more detail. 


\subsection{The model $\mathrm{RH}$ problem}

The model RH problem is not uniquely solvable.

Proposition 2.1 Let $\Psi_{\alpha}$ be a solution of the model RH problem. Then the following hold.

(a) $\operatorname{det} \Psi_{\alpha} \equiv 1$.

(b) For any $\eta \in \mathbb{R}$ (which may depend on $s$ ), we have that $\left(\begin{array}{ll}1 & 0 \\ \eta & 1\end{array}\right) \Psi_{\alpha}$ also solves the model $R H$ problem.

(c) Any two solutions are related as in part (b), i.e., if $\Psi_{\alpha}^{(1)}$ and $\Psi_{\alpha}^{(2)}$ are two solutions of the model $R H$ problem, then $\Psi_{\alpha}^{(2)}=\left(\begin{array}{ll}1 & 0 \\ \eta & 1\end{array}\right) \Psi_{\alpha}^{(1)}$ for some $\eta=\eta(s)$.

Proof. (a) We have that $\operatorname{det} \Psi_{\alpha}$ is analytic in $\mathbb{C} \backslash\{0\}$, since all jump matrices have determinant one. In case $\alpha<0$ we get from condition $(\mathrm{d})$ of the $\mathrm{RH}$ problem that $\operatorname{det} \Psi_{\alpha}(\zeta)=O\left(|\zeta|^{2 \alpha}\right)$ as $\zeta \rightarrow 0$. Since $2 \alpha>-1$ it follows that the singularity at the origin is removable. In case $\alpha \geq 0$ we find from condition (d) of the RH problem that $\operatorname{det} \Psi_{\alpha}(\zeta)=O(1)$ as $\zeta \rightarrow 0$ in $\Omega_{1} \cup \Omega_{4}$. Thus the singularity at the origin cannot be a pole. Since $\operatorname{det} \Psi_{\alpha}=O\left(|\zeta|^{-2 \alpha}\right)$ as $\zeta \rightarrow 0$, it cannot be an essential singularity either and therefore the singularity at the origin is removable also in this case. Hence det $\Psi_{\alpha}$ is entire. From condition (c) of the RH problem it follows that $\operatorname{det} \Psi_{\alpha}(\zeta) \rightarrow 1$ as $\zeta \rightarrow \infty$, and so part (a) of the proposition follows from Liouville's theorem.

(b) It is clear that $\left(\begin{array}{ll}1 & 0 \\ \eta & 1\end{array}\right) \Psi_{\alpha}$ satisfies the conditions (a), (b), and (d) of the model RH problem. To establish (c) it is enough to observe that

$$
\begin{aligned}
\left(\begin{array}{ll}
1 & 0 \\
\eta & 1
\end{array}\right) \zeta^{-\sigma_{3} / 4} \frac{1}{\sqrt{2}}\left(\begin{array}{ll}
1 & i \\
i & 1
\end{array}\right) & =\zeta^{-\sigma_{3} / 4} \frac{1}{\sqrt{2}}\left(\begin{array}{ll}
1 & i \\
i & 1
\end{array}\right)\left(I+\frac{\eta}{2 \zeta^{1 / 2}}\left(\begin{array}{cc}
-i & 1 \\
1 & i
\end{array}\right)\right) \\
& =\zeta^{-\sigma_{3} / 4} \frac{1}{\sqrt{2}}\left(\begin{array}{ll}
1 & i \\
i & 1
\end{array}\right)\left(I+O\left(1 / \zeta^{1 / 2}\right)\right)
\end{aligned}
$$

as $\zeta \rightarrow \infty$.

(c) In view of part (a) we know that $\Psi_{\alpha}^{(1)}$ is invertible. Then $\Psi_{\alpha}^{(2)}\left(\Psi_{\alpha}^{(1)}\right)^{-1}$ is analytic in $\mathbb{C} \backslash\{0\}$ and, by arguments similar to those in the proof of part (a), the singularity at the origin is removable. As $\zeta \rightarrow \infty$ we get from condition (c) of the model RH problem that

$$
\begin{aligned}
\Psi_{\alpha}^{(2)}(\zeta)\left(\Psi_{\alpha}^{(1)}(\zeta)\right)^{-1} & =\zeta^{-\sigma_{3} / 4} \frac{1}{\sqrt{2}}\left(\begin{array}{cc}
1 & i \\
i & 1
\end{array}\right)\left(I+O\left(\zeta^{-1 / 2}\right)\right) \frac{1}{\sqrt{2}}\left(\begin{array}{cc}
1 & -i \\
-i & 1
\end{array}\right) \zeta^{\sigma_{3} / 4} \\
& =I+O\left(\begin{array}{cc}
\zeta^{-1 / 2} & \zeta^{-1} \\
1 & \zeta^{-1 / 2}
\end{array}\right)
\end{aligned}
$$

The statement now follows from Liouville's theorem.

In the following we will need more information about the behavior at the origin of functions satisfying properties (a), (b), and (d) of the model RH problem. The following result is similar to Proposition 2.3 in [9].

Proposition 2.2 Let $\Psi$ satisfy conditions (a), (b), and (d) of the RH problem for $\Psi_{\alpha}$. Then, with all branches being principal, the following hold. 
- If $\alpha-\frac{1}{2} \notin \mathbb{N}_{0}$, there exist an analytic matrix-valued function $E$ and constant matrices $A_{j}$ such that

$$
\Psi(\zeta)=E(\zeta) \zeta^{\alpha \sigma_{3}} A_{j}, \quad \text { for } \zeta \in \Omega_{j}
$$

Letting $v_{j}$ denote the jump matrix for $\Psi$ on $\Sigma_{j}$, we have

$$
A_{1}=A_{4} v_{1}, \quad A_{1}=A_{2} v_{2}, \quad A_{3}=A_{4} v_{4}
$$

and

$$
A_{2}=\left(\begin{array}{cc}
\frac{1}{2 \cos \alpha \pi} & \frac{1}{2 \cos \alpha \pi} \\
-e^{\alpha \pi i} & e^{-\alpha \pi i}
\end{array}\right)
$$

- If $\alpha-\frac{1}{2} \in \mathbb{N}_{0}$, then $\Psi$ has logarithmic behavior at the origin: There exist an analytic matrix-valued function $E$ and constant matrices $A_{j}$ such that

$$
\Psi(\zeta)=E(\zeta)\left(\begin{array}{cc}
\zeta^{\alpha} & \frac{1}{\pi} \zeta^{\alpha} \log \zeta \\
0 & \zeta^{-\alpha}
\end{array}\right) A_{j}, \quad \text { for } \zeta \in \Omega_{j} .
$$

Letting $v_{j}$ denote the jump matrix for $\Psi$ on $\Sigma_{j}$, we now have

$$
A_{1}=A_{4} v_{1}, \quad A_{1}=A_{2} v_{2}, \quad A_{3}=A_{4} v_{4}
$$

and

$$
A_{2}=\left(\begin{array}{cc}
0 & e^{3 \pi i / 4} \\
e^{\pi i / 4} & e^{\pi i / 4}
\end{array}\right)
$$

- In all cases it holds that $\operatorname{det} A_{j}=1$ and

$$
\left(A_{1}\right)_{21}=\left(A_{4}\right)_{21}=0
$$

Proof. The statement (2.10) is an immediate consequence of the explicit formulas for the $A_{j}$ 's.

Consider the case $\alpha-\frac{1}{2} \notin \mathbb{N}_{0}$. Define $E$ by (2.4), i.e., let

$$
E(\zeta)=\Psi(\zeta) A_{j}^{-1} \zeta^{-\alpha \sigma_{3}}, \quad \text { for } \zeta \in \Omega_{j},
$$

with $A_{j}$ as in (2.5), (2.6). Then $E$ is analytic in $\mathbb{C} \backslash \Sigma$. We now show that $E$ is indeed entire. The relations (2.5) and the condition (b) of the model RH problem imply that $E$ is analytic also on $\Sigma_{1} \cup \Sigma_{2} \cup \Sigma_{4}$. Moreover, on $\Sigma_{3}$

$$
E_{-}^{-1}(\zeta) E_{+}(\zeta)=\zeta_{-}^{\alpha \sigma_{3}} A_{3} v_{3} A_{2}^{-1} \zeta_{+}^{-\alpha \sigma_{3}}
$$

Now, by (2.5), (2.6), and straightforward computation

$$
A_{3} v_{3} A_{2}^{-1}=A_{2} v_{2} v_{1}^{-1} v_{4} v_{3} A_{2}^{-1}=e^{2 \alpha \pi i \sigma_{3}}=\zeta_{-}^{-\alpha \sigma_{3}} \zeta_{+}^{\alpha \sigma_{3}} .
$$


Thus, $E$ is analytic also on $\Sigma_{3}$, and therefore in $\mathbb{C} \backslash\{0\}$.

We next show that the singularity at 0 is removable. If $\alpha<0$, we see from (2.11) and the condition (d) of the model $\mathrm{RH}$ problem, that as $\zeta \rightarrow 0$

$$
E(\zeta)=O\left(\begin{array}{ll}
|\zeta|^{\alpha} & |\zeta|^{\alpha} \\
|\zeta|^{\alpha} & |\zeta|^{\alpha}
\end{array}\right) O\left(\begin{array}{cc}
1 & 1 \\
1 & 1
\end{array}\right) O\left(\begin{array}{cc}
|\zeta|^{-\alpha} & 0 \\
0 & |\zeta|^{\alpha}
\end{array}\right)=O\left(\begin{array}{cc}
1 & |\zeta|^{2 \alpha} \\
1 & |\zeta|^{2 \alpha}
\end{array}\right)
$$

so (since $2 \alpha>-1$ ) the isolated singularity at 0 is indeed removable. If $\alpha \geq 0$ and $\zeta \rightarrow 0$ in $\Omega_{1}$ we find in the same way (also using $\left(A_{1}\right)_{21}=0$ ) that

$$
E(\zeta)=O\left(\begin{array}{ll}
|\zeta|^{\alpha} & |\zeta|^{-\alpha} \\
|\zeta|^{\alpha} & |\zeta|^{-\alpha}
\end{array}\right) O\left(\begin{array}{cc}
1 & 1 \\
0 & 1
\end{array}\right) O\left(\begin{array}{cc}
|\zeta|^{-\alpha} & 0 \\
0 & |\zeta|^{\alpha}
\end{array}\right)=O\left(\begin{array}{ll}
1 & 1 \\
1 & 1
\end{array}\right)
$$

so that $E$ is bounded near 0 in $\Omega_{1}$ and thus 0 cannot be a pole. Since 0 cannot be an essential singularity either, we conclude that the singularity is indeed removable.

In case $\alpha-\frac{1}{2} \in \mathbb{N}_{0}$ the proof is almost identical, only now the equation (2.12) is replaced by

$$
\begin{aligned}
A_{3} v_{3} A_{2}^{-1} & =A_{2} v_{2} v_{1}^{-1} v_{4} v_{3} A_{2}^{-1}=\left(\begin{array}{cc}
-1 & -2 i \\
0 & -1
\end{array}\right) \\
& =\left(\begin{array}{cc}
\zeta^{-\alpha} & -\frac{1}{\pi} \zeta^{\alpha} \log \zeta \\
0 & \zeta^{\alpha}
\end{array}\right)_{-}\left(\begin{array}{cc}
\zeta^{\alpha} & \frac{1}{\pi} \zeta^{\alpha} \log \zeta \\
0 & \zeta^{-\alpha}
\end{array}\right)_{+}
\end{aligned}
$$

\subsection{Existence of solution to the model RH problem}

We will need that for $s \in \mathbb{R}$ the model $\mathrm{RH}$ problem indeed has a solution. To prove existence of a solution to the model $\mathrm{RH}$ problem it suffices to prove existence of a (unique) solution $\Psi_{\alpha}^{(s p e c)}$ to the special $\mathrm{RH}$ problem obtained when the asymptotics (c) at infinity is replaced by the following stronger condition

$$
\Psi_{\alpha}^{(s p e c)}(\zeta)=(I+O(1 / \zeta)) \zeta^{-\sigma_{3} / 4} \frac{1}{\sqrt{2}}\left(\begin{array}{ll}
1 & i \\
i & 1
\end{array}\right) e^{-\left(\frac{2}{3} \zeta^{3 / 2}+s \zeta^{1 / 2}\right) \sigma_{3}}
$$

as $\zeta \rightarrow \infty$.

A key element in the proof of unique solvability of the $\mathrm{RH}$ problem for $\Psi_{\alpha}^{(s p e c)}$ is the following vanishing lemma (cf. [25]).

Proposition 2.3 (vanishing lemma) Let $\alpha>-1 / 2, s \in \mathbb{R}$, and put $\theta(\zeta)=\theta(\zeta ; s)=$ $\frac{2}{3} \zeta^{3 / 2}+s \zeta^{1 / 2}$. Suppose that $F_{\alpha}$ satisfies the conditions $(\mathrm{a}),(\mathrm{b})$, and $(\mathrm{d})$ in the $R H$ problem for $\Psi_{\alpha}$ but, instead of condition (c), has the following behavior at infinity:

$$
F_{\alpha}(\zeta)=O(1 / \zeta) \zeta^{-\sigma_{3} / 4} \frac{1}{\sqrt{2}}\left(\begin{array}{cc}
1 & i \\
i & 1
\end{array}\right) e^{-\theta(\zeta) \sigma_{3}}
$$

as $\zeta \rightarrow \infty$. Then $F_{\alpha} \equiv 0$. 
Proof. The ideas of the proof are similar in spirit to those in Deift et al. [14. Let $G_{\alpha}$ be defined as follows:

$$
G_{\alpha}(\zeta)= \begin{cases}F_{\alpha}(\zeta) e^{\theta(\zeta) \sigma_{3}}\left(\begin{array}{cc}
0 & -1 \\
1 & 0
\end{array}\right), & \text { for } \zeta \in \Omega_{1}, \\
F_{\alpha}(\zeta) e^{\theta(\zeta) \sigma_{3}}\left(\begin{array}{cc}
1 & 0 \\
e^{2 \alpha \pi i} e^{2 \theta(\zeta)} & 1
\end{array}\right)\left(\begin{array}{cc}
0 & -1 \\
1 & 0
\end{array}\right), & \text { for } \zeta \in \Omega_{2}, \\
F_{\alpha}(\zeta) e^{\theta(\zeta) \sigma_{3}}\left(\begin{array}{cc}
1 & 0 \\
-e^{-2 \alpha \pi i} e^{2 \theta(\zeta)} & 1
\end{array}\right), & \text { for } \zeta \in \Omega_{3}, \\
F_{\alpha}(\zeta) e^{\theta(\zeta) \sigma_{3}}, & \text { for } \zeta \in \Omega_{4} .\end{cases}
$$

Then $G_{\alpha}$ satisfies the following $\mathrm{RH}$ problem.

\section{Riemann-Hilbert problem for $G_{\alpha}$}

(a) $G_{\alpha}: \mathbb{C} \backslash \mathbb{R} \rightarrow \mathbb{C}^{2 \times 2}$ is analytic.

(b) $G_{\alpha,+}(\zeta)=G_{\alpha,-}(\zeta) v_{G_{\alpha}}(\zeta)$ for $\zeta \in \mathbb{R} \backslash\{0\}$, where

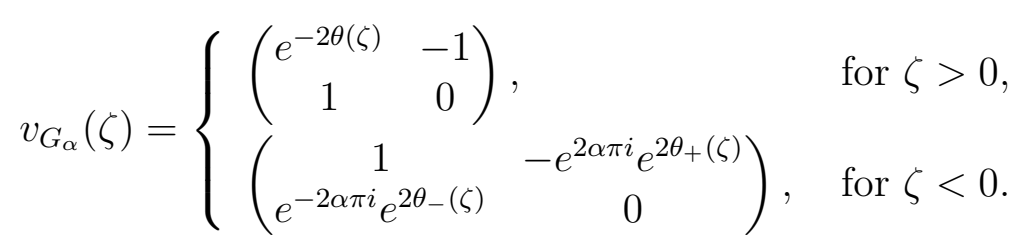

(c) $G_{\alpha}(\zeta)=O\left(\zeta^{-3 / 4}\right) \quad$ as $\zeta \rightarrow \infty$.

(d) $G_{\alpha}$ has the following behavior near the origin: If $\alpha<0$,

$$
G_{\alpha}(\zeta)=O\left(\begin{array}{ll}
|\zeta|^{\alpha} & |\zeta|^{\alpha} \\
|\zeta|^{\alpha} & |\zeta|^{\alpha}
\end{array}\right), \quad \text { as } \zeta \rightarrow 0
$$

and if $\alpha \geq 0$,

$$
G_{\alpha}(\zeta)= \begin{cases}O\left(\begin{array}{ll}
|\zeta|^{-\alpha} & |\zeta|^{\alpha} \\
|\zeta|^{-\alpha} & |\zeta|^{\alpha}
\end{array}\right) \quad \text { as } \zeta \rightarrow 0, \operatorname{Im} \zeta>0, \\
O\left(\begin{array}{ll}
|\zeta|^{\alpha} & |\zeta|^{-\alpha} \\
|\zeta|^{\alpha} & |\zeta|^{-\alpha}
\end{array}\right) \quad \text { as } \zeta \rightarrow 0, \operatorname{Im} \zeta<0 .\end{cases}
$$

The jumps in (b) follow from straightforward computations which uses that $\theta_{+}(\zeta)+$ $\theta_{-}(\zeta)=0$ for $\zeta<0$. The behavior (c) of $G_{\alpha}$ at infinity (uniformly in each sector) follows directly from (2.15), (2.16), and the fact that $\operatorname{Re} \theta(\zeta)<0$ for $\zeta \in \Omega_{2} \cup \Omega_{3}$. The behavior (2.18) at the origin is immediate from the condition (d) of the $\mathrm{RH}$ problem for $F_{\alpha}$, and so is the behavior (2.19) if $\zeta \rightarrow 0$ with $\zeta \in \Omega_{1} \cup \Omega_{4}$. To prove (2.19) if $\zeta \rightarrow 0$ with $\zeta \in \Omega_{2} \cup \Omega_{3}$, we need Proposition 2.2. Consider first the case $\alpha-\frac{1}{2} \notin \mathbb{N}_{0}$ and $\zeta \in \Omega_{2}$. 
Then we have, using (2.16), (2.4), (2.5), and (2.10)

$$
\begin{aligned}
G_{\alpha}(\zeta) & =F_{\alpha}(\zeta) e^{\theta(\zeta) \sigma_{3}}\left(\begin{array}{cc}
1 & 0 \\
e^{2 \alpha \pi i} e^{2 \theta(\zeta)} & 1
\end{array}\right)\left(\begin{array}{cc}
0 & -1 \\
1 & 0
\end{array}\right) \\
& =E(\zeta) \zeta^{\alpha \sigma_{3}} A_{2}\left(\begin{array}{cc}
1 & 0 \\
e^{2 \alpha \pi i} & 1
\end{array}\right) e^{\theta(\zeta) \sigma_{3}}\left(\begin{array}{cc}
0 & -1 \\
1 & 0
\end{array}\right) \\
& =E(\zeta) \zeta^{\alpha \sigma_{3}} A_{2}\left(\begin{array}{cc}
1 & 0 \\
e^{2 \alpha \pi i} & 1
\end{array}\right)\left(\begin{array}{cc}
0 & -1 \\
1 & 0
\end{array}\right) e^{-\theta(\zeta) \sigma_{3}} \\
& =E(\zeta) \zeta^{\alpha \sigma_{3}} A_{1}\left(\begin{array}{cc}
0 & -1 \\
1 & 0
\end{array}\right) e^{-\theta(\zeta) \sigma_{3}}=E(\zeta) \zeta^{\alpha \sigma_{3}}\left(\begin{array}{cc}
* & * \\
0 & *
\end{array}\right)\left(\begin{array}{cc}
0 & -1 \\
1 & 0
\end{array}\right) e^{-\theta(\zeta) \sigma_{3}},
\end{aligned}
$$

where $*$ denotes an unspecified constant. Using the boundedness of $E$ and $\theta$ at the origin, we find $(2.19)$ as $\zeta \rightarrow 0$ in the sector $\Omega_{2}$. The case $\zeta \in \Omega_{3}$ is treated similarly. Using (2.7), (2.8) instead of (2.4), (2.5), the same argument works in case $\alpha-\frac{1}{2} \in \mathbb{N}_{0}$. Note that in spite of the logarithmic entry in (2.8), there are no logarithmic entries in (2.19).

Introduce the auxiliary matrix-valued function

$$
H_{\alpha}(\zeta)=G_{\alpha}(\zeta)\left(G_{\alpha}(\bar{\zeta})\right)^{*}, \quad \zeta \in \mathbb{C} \backslash \mathbb{R}
$$

Then $H_{\alpha}$ is analytic and

$$
H_{\alpha}(\zeta)=O\left(\zeta^{-3 / 2}\right), \quad \text { as } \zeta \rightarrow \infty
$$

From the condition (d) in the RH problem for $G_{\alpha}$ it follows that $H_{\alpha}$ has the following behavior near the origin:

$$
H_{\alpha}(\zeta)= \begin{cases}O\left(\begin{array}{ll}
|\zeta|^{2 \alpha} & |\zeta|^{2 \alpha} \\
|\zeta|^{2 \alpha} & |\zeta|^{2 \alpha}
\end{array}\right) & \text { as } \zeta \rightarrow 0, \text { in case } \alpha<0 \\
O\left(\begin{array}{ll}
1 & 1 \\
1 & 1
\end{array}\right) & \text { as } \zeta \rightarrow 0, \text { in case } \alpha \geq 0\end{cases}
$$

Since $\alpha>-1 / 2$, we see from (2.21) and (2.22) that each entry of $H_{\alpha,+}$ is integrable over the real line, and by Cauchy's theorem and (2.21)

$$
\int_{\mathbb{R}} H_{\alpha,+}(\zeta) d \zeta=0 .
$$

That is, by (2.20),

$$
\int_{\mathbb{R}} G_{\alpha,+}(\zeta)\left(G_{\alpha,-}(\zeta)\right)^{*} d \zeta=0 .
$$

Adding (2.24) to its Hermitian conjugate and using (2.17) we obtain

$$
\begin{aligned}
0 & =\int_{\mathbb{R}} G_{\alpha,-}(\zeta)\left[v_{G_{\alpha}}(\zeta)+\left(v_{G_{\alpha}}(\zeta)\right)^{*}\right]\left(G_{\alpha,-}(\zeta)\right)^{*} d \zeta \\
& =\int_{-\infty}^{0} G_{\alpha,-}(\zeta)\left(\begin{array}{ll}
2 & 0 \\
0 & 0
\end{array}\right)\left(G_{\alpha,-}(\zeta)\right)^{*} d \zeta+\int_{0}^{+\infty} G_{\alpha,-}(\zeta)\left(\begin{array}{cc}
2 e^{-2 \theta(\zeta)} & 0 \\
0 & 0
\end{array}\right)\left(G_{\alpha,-}(\zeta)\right)^{*} d \zeta .
\end{aligned}
$$


Here we also used that $\theta_{+}(\zeta)=-\theta_{-}(\zeta) \in i \mathbb{R}$ for $\zeta<0$, which holds because $s$ is real. The identity (2.25) implies that the first column of $G_{\alpha,-}$ vanishes identically on $\mathbb{R}$. Thus, in view of the form of the jump matrix in (2.17), the second column of $G_{\alpha,+}$ vanishes identically on $\mathbb{R}$ as well. It follows that the first column of $G_{\alpha}$ vanishes identically in the lower half-plane, and the second column vanishes identically in the upper half-plane.

To prove that the full matrix $G_{\alpha}$ vanishes identically in both half-planes, we shall use a Phragmen-Lindelöf type theorem due to Carlson [7, 41]. Define for $j=1,2$,

$$
g_{j}(\zeta)= \begin{cases}\left(G_{\alpha}\right)_{j 1}(\zeta), & \text { for } \operatorname{Im} \zeta>0, \\ \left(G_{\alpha}\right)_{j 2}(\zeta), & \text { for } \operatorname{Im} \zeta<0 .\end{cases}
$$

The conditions of the RH problem for $G_{\alpha}$ yield that both $g_{1}$ and $g_{2}$ have analytic continuation across $(0, \infty)$ and that they are both solutions of the following scalar $\mathrm{RH}$ problem.

\section{Riemann-Hilbert problem for $g$}

- $g: \mathbb{C} \backslash(-\infty, 0] \rightarrow \mathbb{C}$ is analytic with jump

$$
g_{+}(\zeta)=g_{-}(\zeta) e^{-2 \alpha \pi i} e^{2 \theta_{-}(\zeta)}, \quad \text { for } \zeta \in(-\infty, 0) .
$$

- $g(\zeta)=O\left(\zeta^{-3 / 4}\right)$ as $\zeta \rightarrow \infty$.

- $g(\zeta)=O\left(|\zeta|^{-|\alpha|}\right)$ as $\zeta \rightarrow 0$.

We are going to prove that this $\mathrm{RH}$ problem has only the trivial solution.

Let $g$ be any solution and define $\hat{g}$ by

$$
\hat{g}(\zeta)= \begin{cases}g\left(\zeta^{2}\right), & \text { if } \operatorname{Re} \zeta>0, \\ g\left(\zeta^{2}\right) e^{-2 \alpha \pi i} e^{-2\left(\frac{2}{3} \zeta^{3}+s \zeta\right),} & \text { if } \operatorname{Re} \zeta<0, \operatorname{Im} \zeta>0, \\ g\left(\zeta^{2}\right) e^{2 \alpha \pi i} e^{-2\left(\frac{2}{3} \zeta^{3}+s \zeta\right),} & \text { if } \operatorname{Re} \zeta<0, \operatorname{Im} \zeta<0 .\end{cases}
$$

The jump property (2.27) ensures that $\hat{g}$ is analytic across the imaginary axis.

Now define

$$
h(\zeta)=\left(\frac{\zeta}{1+\zeta}\right)^{\frac{8}{3}|\alpha|} \hat{g}\left(\zeta^{4 / 3}\right), \quad \text { for } \operatorname{Re} \zeta \geq 0,
$$

with (as usual) the principal branches of the fractional powers. Then it can be checked that $h$ is analytic in $\operatorname{Re} \zeta>0$, bounded for $\operatorname{Re} \zeta \geq 0$, and satisfies

$$
|h(\zeta)| \leq C e^{-c|\zeta|^{4}}, \quad \text { if } \zeta \in i \mathbb{R},
$$

for some positive constants $c$ and $C$. Hence, by Carlson's theorem, $h \equiv 0$ in $\operatorname{Re} \zeta \geq 0$. Therefore $g \equiv 0$, and so $g_{1}$ and $g_{2}$ are both identically zero. It follows that the full matrix $G_{\alpha}$ vanishes identically in both half-planes.

Thus $F_{\alpha} \equiv 0$ by (2.16), and this completes the proof of the proposition.

We now show how (unique) solvability of the RH problem for $\Psi_{\alpha}^{(s p e c)}$ can be deduced from the above vanishing lemma. 
Proposition 2.4 The RH problem for $\Psi_{\alpha}^{(\text {spec })}$ has a unique solution for every $s \in \mathbb{R}$.

Proof. The idea of the proof is this: Given a solution $\Psi_{\alpha}^{(s p e c)}$ to the above RH problem, we show how to construct a solution $m_{\alpha}$ to a certain normalized RH problem (i.e., $m_{\alpha}(\zeta) \rightarrow I$ as $\left.\zeta \rightarrow \infty\right)$ characterized by a jump matrix $v$ on a contour $\widetilde{\Sigma}$, and conversely. To prove the proposition it therefore suffices to prove (unique) solvability of the normalized RH problem. This can be done by utilizing the basic relationship between normalized RH problems and singular integral equations. We recall briefly, in our setting, some standard facts regarding this relationship. For further details, and proofs, the reader is referred to the papers [17, 18, 19, 44], and to the appendix of [32].

Let $C$ denote the Cauchy operator

$$
C h(\zeta)=\frac{1}{2 \pi i} \int_{\widetilde{\Sigma}} \frac{h(s)}{s-\zeta} d s, \quad h \in L^{2}(\widetilde{\Sigma}), \quad \zeta \in \mathbb{C} \backslash \widetilde{\Sigma}
$$

and denote by $C_{ \pm} h(\zeta), \zeta \in \widetilde{\Sigma}$, the limits of $C h\left(\zeta^{\prime}\right)$ as $\zeta^{\prime} \rightarrow \zeta$ from the $( \pm)$-side of $\widetilde{\Sigma}$. The operators $C_{ \pm}$are bounded on $L^{2}(\widetilde{\Sigma})$. Let

$$
v(\zeta)=\left(v_{-}(\zeta)\right)^{-1} v_{+}(\zeta), \quad \zeta \in \widetilde{\Sigma}
$$

be a pointwise factorization of $v(\zeta)$ with $v_{ \pm}(\zeta) \in \mathrm{GL}(2, \mathbb{C})$, and define $\omega_{ \pm}$through

$$
v_{ \pm}(\zeta)=I \pm \omega_{ \pm}(\zeta), \quad \zeta \in \widetilde{\Sigma}
$$

Our choice of factorization will imply that $\omega_{ \pm} \in L^{2}(\widetilde{\Sigma}) \cap L^{\infty}(\widetilde{\Sigma})$. The singular integral operator $C_{\omega}: L^{2}(\widetilde{\Sigma}) \rightarrow L^{2}(\widetilde{\Sigma})$, defined by

$$
C_{\omega} h=C_{+}\left(h \omega_{-}\right)+C_{-}\left(h \omega_{+}\right), \quad h \in L^{2}(\widetilde{\Sigma}),
$$

is then bounded on $L^{2}(\widetilde{\Sigma})$. Moreover, it makes sense to study the singular integral equation

$$
\left(1-C_{\omega}\right) \mu=I
$$

for $\mu \in I+L^{2}(\widetilde{\Sigma})$. For if we write $\mu=I+h$, then (2.34) takes the form

$$
\left(1-C_{\omega}\right) h=C_{\omega} I \in L^{2}(\widetilde{\Sigma}) .
$$

Suppose that $\mu \in I+L^{2}(\widetilde{\Sigma})$ is a solution of (2.34) . Then, indeed

$$
m_{\alpha}(\zeta)=I+C\left(\mu\left(\omega_{+}+\omega_{-}\right)\right)(\zeta), \quad \zeta \in \mathbb{C} \backslash \widetilde{\Sigma},
$$

solves the normalized $\mathrm{RH}$ problem. Thus, if we can prove that the operator $1-C_{\omega}$ is a bijection in $L^{2}(\widetilde{\Sigma})$, then solvability of the $\mathrm{RH}$ problem for $m_{\alpha}$, and hence of that for $\Psi_{\alpha}^{(s p e c)}$, has been established. Bijectivity of $1-C_{\omega}$ in $L^{2}(\widetilde{\Sigma})$ is proved in two steps. We first show that, for an appropriate choice of $\omega=\left(\omega_{-}, \omega_{+}\right)$in the above factorization, $1-C_{\omega}$ is Fredholm in $L^{2}(\widetilde{\Sigma})$ with index 0 . Second, we show that the kernel of $1-C_{\omega}$ is trivial. Now, it is a standard fact that ker $\left(1-C_{\omega}\right)=\{0\}$ if and only if the associated homogeneous $\mathrm{RH}$ problem (for say $m_{\alpha}^{0}$ ) has only the trivial solution. But the explicit relation between $\Psi_{\alpha}^{(s p e c)}$ and $m_{\alpha}$ also establishes a relation between solutions $F_{\alpha}$ and $m_{\alpha}^{0}$ 


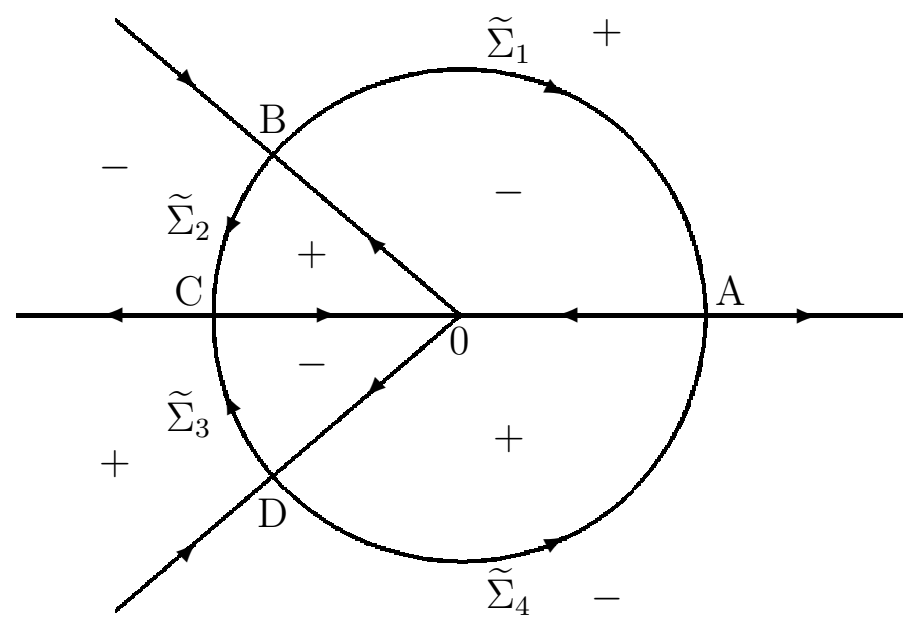

Figure 2: Contour for the RH problem for $m_{\alpha}$.

of the associated homogeneous $\mathrm{RH}$ problems. In view of Proposition 2.3, which states that $F_{\alpha} \equiv 0$, the second step has thus already been accomplished.

We now establish the above mentioned relation between $\Psi_{\alpha}^{(s p e c)}$ and $m_{\alpha}$, derive the $\mathrm{RH}$ problem satisfied by $m_{\alpha}$, and finally show that a factorization of $v$ may be chosen so that $1-C_{\omega}$ is Fredholm with index 0, cf. [25].

Let $\mathbb{D}=\{\zeta \in \mathbb{C}|| \zeta \mid<1\}$. Set $\theta(\zeta)=\frac{2}{3} \zeta^{3 / 2}+s \zeta^{1 / 2}$ and

$$
m_{\alpha}(\zeta)= \begin{cases}\Psi_{\alpha}^{(s p e c)}(\zeta) A_{j}^{-1}\left(\begin{array}{cc}
\zeta^{-\alpha} & -\frac{\kappa_{\alpha}}{\pi} \zeta^{\alpha} \log \zeta \\
0 & \zeta^{\alpha}
\end{array}\right), & \text { for } \zeta \in \Omega_{j} \cap \mathbb{D} \\
\Psi_{\alpha}^{(s p e c)}(\zeta) e^{\theta(\zeta) \sigma_{3}} \frac{1}{\sqrt{2}}\left(\begin{array}{cc}
1 & -i \\
-i & 1
\end{array}\right) \zeta^{\sigma_{3} / 4}, & \text { for } \zeta \in \Omega_{j} \cap \overline{\mathbb{D}}^{c}\end{cases}
$$

with $\left\{A_{j}\right\}_{j=1}^{4}$ being the matrices in Proposition 2.2, and where $\kappa_{\alpha}=1$ if $\alpha-1 / 2 \in \mathbb{N}_{0}$ and 0 otherwise. By Proposition 2.2 it follows that $m_{\alpha}$ is analytic in $\mathbb{D}$. Let $\widetilde{\Sigma}=\Sigma \cup \partial \mathbb{D}$ and orient the components of $\widetilde{\Sigma}$ as in Figure 2. This makes $\widetilde{\Sigma}$ a complete contour, meaning that $\mathbb{C} \backslash \widetilde{\Sigma}$ can be expressed as the union of two disjoint sets, $\mathbb{C} \backslash \widetilde{\Sigma}=\Omega_{+} \cup \Omega_{-}, \Omega_{+} \cap \Omega_{-}=\emptyset$, such that $\widetilde{\Sigma}$ is the positively oriented boundary of $\Omega_{+}$and the negatively oriented boundary of $\Omega_{-}$. Let $\widetilde{\Sigma}_{j}=\Omega_{j} \cap \partial \mathbb{D}$.

Computations show that $m_{\alpha}$ satisfies the following normalized $\mathrm{RH}$ problem. As in Proposition 2.2 we use $v_{j}$ to denote the jump matrix on $\Sigma_{j}$ in the model RH problem.

\section{Riemann-Hilbert problem for $m_{\alpha}$}

- $m_{\alpha}: \mathbb{C} \backslash \widetilde{\Sigma} \rightarrow \mathbb{C}^{2 \times 2}$ is analytic.

- $m_{\alpha,+}(\zeta)=m_{\alpha,-}(\zeta) v(\zeta)$ for $\zeta \in \widetilde{\Sigma}$, where 


$$
v(\zeta)= \begin{cases}I, & \text { for } \zeta \in \widetilde{\Sigma} \cap \mathbb{D}, \\
\zeta^{-\sigma_{3} / 4} \frac{1}{\sqrt{2}}\left(\begin{array}{ll}
1 & i \\
i & 1
\end{array}\right) e^{-\theta \sigma_{3}} v_{j} e^{\theta \sigma_{3}} \frac{1}{\sqrt{2}}\left(\begin{array}{cc}
1 & -i \\
-i & 1
\end{array}\right) \zeta^{\sigma_{3} / 4}, & \text { for } \zeta \in \Sigma_{j} \cap \overline{\mathbb{D}}^{c}, j \in\{1,2,4\} \\
I, & \text { for } \zeta \in \Sigma_{3} \cap \overline{\mathbb{D}}^{c}, \\
\left(\begin{array}{cc}
\zeta^{\alpha} & \frac{\kappa_{\alpha}}{\pi} \zeta^{\alpha} \log \zeta \\
0 & \zeta^{-\alpha}
\end{array}\right) A_{j} e^{\theta \sigma_{3}} \frac{1}{\sqrt{2}}\left(\begin{array}{cc}
1 & -i \\
-i & 1
\end{array}\right) \zeta^{\sigma_{3} / 4}, & \text { for } \zeta \in \widetilde{\Sigma}_{j}, j \in\{1,3\}, \\
\zeta^{-\sigma_{3} / 4} \frac{1}{\sqrt{2}}\left(\begin{array}{ll}
1 & i \\
i & 1
\end{array}\right) e^{-\theta \sigma_{3}} A_{j}^{-1}\left(\begin{array}{cc}
\zeta^{-\alpha} & -\frac{\kappa_{\alpha}}{\pi} \zeta^{\alpha} \log \zeta \\
0 & \zeta^{\alpha}
\end{array}\right), & \text { for } \zeta \in \widetilde{\Sigma}_{j}, j \in\{2,4\} . \\
& \end{cases}
$$

The analyticity of $m_{\alpha}$ on $\Sigma_{3} \cap \overline{\mathbb{D}}^{c}$ follows since $\theta_{+}(\zeta)+\theta_{-}(\zeta)=0$ for $\zeta<0$.

It is important to note that $v(\zeta)-I$ decays exponentially as $\zeta \rightarrow \infty$ along $\widetilde{\Sigma}$. Next observe that, at any of the points $0, A, B, C, D$ of self-intersection of $\widetilde{\Sigma}$ (see Figure 2), precisely four contours come together. At a fixed point of self-intersection, say $P$, order the contours that meet at $P$ counterclockwise, starting from any contour that is oriented outwards from $P$. Denoting the limiting value of the jump matrices over the $j$ th contour at $P$ by $v^{(j)}(P)$, we then have the cyclic relation

$$
v^{(1)}(P)\left(v^{(2)}(P)\right)^{-1} v^{(3)}(P)\left(v^{(4)}(P)\right)^{-1}=I .
$$

This is trivial in case $P=0$, and follows by direct computation in the other cases. We remark that the cyclic relation (2.38) at $C$ is a consequence of the relation (2.12) in the case $\alpha-1 / 2 \notin \mathbb{N}_{0}$, and of (2.13) in the case $\alpha-1 / 2 \in \mathbb{N}_{0}$ (see the proof of Proposition 2.2).

Outside small neighborhoods of the points of self-intersection we choose the trivial factorization $v_{+}=v, v_{-}=I$ in (2.31), so that $\omega_{+}=v-I, \omega_{-}=0$ by (2.32). Using the cyclic relations (2.38), we are then able to choose a factorization of $v$ in the remaining neighborhoods in such a way that $\omega_{+}$is continuous along the boundary of each connected component of $\Omega_{+}$, and similarly, $\omega_{-}$is continuous along the boundary of each connected component of $\Omega_{-}$.

The exponential decay of $v(\zeta)-I$ as $\zeta \rightarrow \infty$ ensures that $\omega_{ \pm} \in L^{2}(\widetilde{\Sigma}) \cap L^{\infty}(\widetilde{\Sigma})$. From this it follows that $1-C_{\omega}$ is Fredholm in $L^{2}(\widetilde{\Sigma})$. Indeed, set

$$
\widetilde{\omega}_{-}=I-v_{-}^{-1}, \quad \widetilde{\omega}_{+}=v_{+}^{-1}-I .
$$

The choice of $\widetilde{\omega}=\left(\widetilde{\omega}_{-}, \widetilde{\omega}_{+}\right)$is motivated by the relations

$$
\widetilde{\omega}_{-} \omega_{-}=\widetilde{\omega}_{-}+\omega_{-}, \quad \widetilde{\omega}_{+} \omega_{+}=-\left(\widetilde{\omega}_{+}+\omega_{+}\right) .
$$

A direct calculation, using $C_{+}-C_{-}=1$ and (2.40), shows that

$$
\left(1-C_{\omega}\right)\left(1-C_{\widetilde{\omega}}\right)=1+T
$$

where

$$
T f=C_{+}\left(\left(C_{-}\left[f\left(\widetilde{\omega}_{+}+\widetilde{\omega}_{-}\right)\right]\right) \omega_{-}\right)+C_{-}\left(\left(C_{+}\left[f\left(\widetilde{\omega}_{+}+\widetilde{\omega}_{-}\right)\right]\right) \omega_{+}\right)
$$


for $f \in L^{2}(\widetilde{\Sigma})$. Standard computations, using continuity of the functions $\omega_{+}$resp. $\omega_{-}$ along the boundary of each connected component of $\Omega_{+}$resp. $\Omega_{-}$, show that $T$ is compact in $L^{2}(\widetilde{\Sigma})$. Similar computations show that $\left(1-C_{\widetilde{\omega}}\right)\left(1-C_{\omega}\right)=1+S$, with $S$ compact in $L^{2}(\widetilde{\Sigma})$. So $1-C_{\widetilde{\omega}}$ is a pseudoinverse for $1-C_{\omega}$, which is therefore Fredholm in $L^{2}(\widetilde{\Sigma})$.

It follows from general theory that the index of the operator $1-C_{\omega}$ equals the winding number of $\operatorname{det} v$ along $\widetilde{\Sigma}$, the latter being defined in the natural way. Now, since $\operatorname{det} v \equiv 1$, this is trivially zero. This completes the proof of Proposition 2.4.

Remark 2.5 The RH problem for $\Psi_{\alpha}^{(\text {spec })}$ is indeed solvable for all $s \in \mathbb{C} \backslash \mathcal{D}$, where $\mathcal{D}$ is

a discrete set in $\mathbb{C}$ (disjoint from $\mathbb{R}$ according to Proposition 2.4 ), and the solution $\Psi_{\alpha}^{(\text {spec })}$ is meromorphic in $s$ with poles in $\mathcal{D}$. To see this, we first observe that the factorization (2.31), (2.32) can be done so that $\omega_{ \pm}$are both analytic in $s$. It follows that $s \mapsto 1-C_{\omega}$ is an analytic map taking values in the Fredholm operators on $L^{2}(\widetilde{\Sigma})$. Since we know that $1-C_{\omega}$ is invertible for $s \in \mathbb{R}$, we then get, by a version of the analytic Fredholm theorem [44], that $\mu$ defined by (2.34) is meromorphic. Thus $m_{\alpha}$ and hence $\Psi_{\alpha}^{(s p e c)}$ is meromorphic in $s$.

\subsection{Some preliminaries on equilibrium measures}

Before we embark on the steepest descent analysis for the $\mathrm{RH}$ problem of Subsection 2.1, we recall certain properties of equilibrium measures, see [11, 40]. We use the following notation:

$$
t=\frac{n}{N}, \quad V_{t}(x)=\frac{1}{t} V(x) .
$$

As explained in the Introduction, we are interested in the case where $n / N \rightarrow 1$ as $n, N \rightarrow$ $\infty$, which means that we are interested in $t$ close to 1 . For every $t$ we consider the energy functional $I_{V_{t}}(\mu)$ as in (1.7), and its minimizer $\mu_{t}$.

The equilibrium measure $d \mu_{t}=\rho_{t} d x$ is characterized by the following Euler-Lagrange variational conditions: There is a constant $l_{t} \in \mathbb{R}$ such that

$$
\begin{aligned}
& 2 \int \log |x-s| \rho_{t}(s) d s-V_{t}(x)+l_{t}=0, \quad x \in \operatorname{supp} \mu_{t}, \\
& 2 \int \log |x-s| \rho_{t}(s) d s-V_{t}(x)+l_{t} \leq 0, \quad x \in \mathbb{R} \backslash \operatorname{supp} \mu_{t} .
\end{aligned}
$$

For $t=1$, we have that the support of $\mu_{V}$ consists of a finite union of disjoint intervals, see [13], say

$$
\operatorname{supp} \mu_{V}=\bigcup_{j=1}^{k}\left[a_{j}, b_{j}\right]
$$

with $a_{1}<b_{1}<a_{2}<\cdots<a_{k}<b_{k}$. Due to the assumption that the density $\rho_{V}$ of $\mu_{V}$ is regular, we have the following proposition.

Proposition 2.6 For every $t$ in an interval around 1, we have that the density $\rho_{t}$ of $\mu_{t}$ is regular, and that $\operatorname{supp} \mu_{t}$ consists of $k$ intervals, say

$$
\operatorname{supp} \mu_{t}=\bigcup_{j=1}^{k}\left[a_{j}(t), b_{j}(t)\right]
$$


with $a_{1}(t)<b_{1}(t)<a_{2}(t)<\cdots<a_{k}(t)<b_{k}(t)$. In this interval around 1 , the functions $t \mapsto a_{j}(t)$ and $t \mapsto b_{j}(t)$ are real analytic with $a_{j}^{\prime}(t)<0$ and $b_{j}^{\prime}(t)>0$.

Proof. See Theorem 1.3 (iii) and Lemma 8.1 of [36].

For the rest of the proof of Theorem 1.1 we shall assume that supp $\mu_{V}$ consists of one interval. In the general case (when supp $\mu_{V}$ consists of $k \geq 2$ intervals) one proceeds analogously, but the parametrix away from the end points given in Subsection 2.5.4 must then instead be constructed with the help of the $\theta$-function of $B$-periods for the twosheeted Riemann surface $y^{2}=\prod_{j=1}^{k}\left[\left(z-a_{j}\right)\left(z-b_{j}\right)\right]$ obtained by gluing together two copies of the slit plane $\mathbb{C} \backslash \bigcup_{j=1}^{k}\left[a_{j}, b_{j}\right]$ in the standard way [14, 37]. Since the formulas will be more complicated in the multi-interval case, but do not contribute to the main issue of the present paper, we chose to give the proof in full for the one-interval case only.

\subsection{Steepest descent analysis}

\subsubsection{Preliminaries}

We assume from now on that $k=1$, so that $\operatorname{supp} \mu_{V}$ consists of one interval which we take as

$$
\operatorname{supp}\left(\mu_{V}\right)=[a, 0], \quad a<0 .
$$

Then there is $\delta_{1}>0$ such that $\mu_{t}$ is supported on one interval $\left[a_{t}, b_{t}\right]$ for every $t \in$ $\left(1-\delta_{1}, 1+\delta_{1}\right)$, and its density $\rho_{t}$ is regular. Hence $\rho_{t}$ is positive on $\left(a_{t}, b_{t}\right)$ and vanishes like a square root at the end points, and it takes the form [14]

$$
\rho_{t}(x)=\frac{1}{2 \pi} \sqrt{\left(b_{t}-x\right)\left(x-a_{t}\right)} h_{t}(x), \quad \text { for } x \in\left[a_{t}, b_{t}\right]
$$

where $h_{t}$ is positive on $\left[a_{t}, b_{t}\right]$, and analytic in the domain of analyticity of $V$. In addition, $h_{t}$ depends analytically on $t \in\left(1-\delta_{1}, 1+\delta_{1}\right)$.

We are going to use the equilibrium measure $\mu_{t}$ in the first transformation of the $\mathrm{RH}$ problem. We remark that in [8, 9, 10, 20] a modified equilibrium measure was used in the steepest descent analysis of a $\mathrm{RH}$ problem at a critical point. It is likely that we could have modified the equilibrium measure in the present situation as well, but the approach with the unmodified $\mu_{t}$ also works, as we will see, and we chose to use it in this paper.

In the one-interval case one can show by explicit computation that

$$
\frac{d}{d t} a_{t}=-\frac{4}{t\left(b_{t}-a_{t}\right) h_{t}\left(a_{t}\right)}, \quad \frac{d}{d t} b_{t}=\frac{4}{t\left(b_{t}-a_{t}\right) h_{t}\left(b_{t}\right)},
$$

which indeed shows that $\frac{d}{d t} a_{t}<0$ and $\frac{d}{d t} b_{t}>0$. It follows that $b_{t}>0$ for $t \in\left(1,1+\delta_{1}\right)$ and $b_{t}<0$ for $t \in\left(1-\delta_{1}, 1\right)$. In both cases we have $a_{t}<0$.

We introduce two functions $\varphi_{t}$ and $\tilde{\varphi}_{t}$ as follows. For $z \in \mathbb{C} \backslash\left(-\infty, b_{t}\right]$ lying in the domain of analyticity of $V$ (which we may restrict to be simply connected, without loss of generality), we put

$$
\varphi_{t}(z)=\frac{1}{2} \int_{b_{t}}^{z}\left(\left(s-b_{t}\right)\left(s-a_{t}\right)\right)^{1 / 2} h_{t}(s) d s
$$


and for $z \in \mathbb{C} \backslash\left[a_{t}, \infty\right)$ also in the domain of analyticity of $V$,

$$
\tilde{\varphi}_{t}(z)=\frac{1}{2} \int_{a_{t}}^{z}\left(\left(s-b_{t}\right)\left(s-a_{t}\right)\right)^{1 / 2} h_{t}(s) d s .
$$

It follows from (2.48) that

$$
\varphi_{t}(z)=\frac{1}{3} \sqrt{-a_{t}} h_{t}\left(b_{t}\right)\left(z-b_{t}\right)^{3 / 2} \chi_{t}(z),
$$

where $\chi_{t}$ is analytic in a neighborhood of $b_{t}$ and $\chi_{t}\left(b_{t}\right)=1$. Taking

$$
f_{t}(z)=\left(\frac{3}{2} \varphi_{t}(z)\right)^{2 / 3}=\left(\frac{1}{2} \sqrt{-a_{t}} h_{t}\left(b_{t}\right)\right)^{2 / 3}\left(z-b_{t}\right) \chi_{t}^{2 / 3}(z)
$$

we see that $f_{t}$ is analytic in a neighborhood of $b_{t}$ with $f_{t}\left(b_{t}\right)=0$,

$$
f_{t}^{\prime}\left(b_{t}\right)=\left(\frac{1}{2} \sqrt{-a_{t}} h_{t}\left(b_{t}\right)\right)^{2 / 3} \neq 0
$$

and $f_{t}(z)$ real for real values of $z$. Hence, in particular,

$$
f_{t}(0)>0 \text {, if } t<1, \quad f_{1}(0)=0, \quad \text { and } \quad f_{t}(0)<0 \text {, if } t>1 .
$$

Moreover, $f_{t} \rightarrow f_{1}$ as $t \rightarrow 1$, uniformly in a neighborhood of 0 . We choose a small disc $U^{(0)}$ around 0 and $\delta_{2}>0$ sufficiently small, so that $f_{t}$ is a conformal map from $U^{(0)}$ onto a convex neighborhood of 0 for every $t \in\left(1-\delta_{2}, 1+\delta_{2}\right)$.

Similarly, there exists a disc $U^{(a)}$ centered at $a<0$, and a $\delta_{3}>0$, so that

$$
\tilde{f}_{t}(z)=\left(\frac{3}{2} \tilde{\varphi}_{t}(z)\right)^{2 / 3}
$$

is a conformal map from $U^{(a)}$ onto a convex neighborhood of 0 for every $t \in\left(1-\delta_{3}, 1+\delta_{3}\right)$.

We let $\delta_{0}=\min \left(\delta_{1}, \delta_{2}, \delta_{3}\right)$ and we fix $t \in\left(1-\delta_{0}, 1+\delta_{0}\right)$. In what follows we also take the neighborhoods $U^{(0)}$ and $U^{(a)}$ as above.

\subsubsection{First transformation $Y \mapsto T$}

We introduce the so-called $g$-function:

$$
g_{t}(z)=\int \log (z-s) d \mu_{t}(s)=\int \log (z-s) \rho_{t}(s) d s, \quad z \in \mathbb{C} \backslash\left(-\infty, b_{t}\right],
$$

where $\log$ denotes the principal branch. Then $g_{t}$ is analytic in $\mathbb{C} \backslash\left(-\infty, b_{t}\right]$. Define $T$ by

$$
T(z)=e^{\frac{1}{2} n l_{t} \sigma_{3}} Y(z) e^{-\frac{1}{2} n l_{t} \sigma_{3}} e^{-n g_{t}(z) \sigma_{3}}, \quad z \in \mathbb{C} \backslash \mathbb{R},
$$

where $l_{t}$ is the constant from (2.44)-(2.45). By a straightforward calculation it then follows that $T$ has the following jump matrix $v_{T}$ on $\mathbb{R}$ (oriented from left to right):

$$
v_{T}(x)=\left(\begin{array}{cc}
e^{-n\left(g_{t,+}(x)-g_{t,-}(x)\right)} & |x|^{2 \alpha} e^{n\left(g_{t,+}(x)+g_{t,-}(x)-V_{t}(x)+l_{t}\right)} \\
0 & e^{n\left(g_{t,+}(x)-g_{t,-}(x)\right)}
\end{array}\right) .
$$

Because of the identities, see [11, 14],

$$
\begin{array}{ll}
g_{t,+}(x)+g_{t,-}(x)-V_{t}(x)+l_{t}=-2 \varphi_{t}(x), & \text { for } x>b_{t}, \\
g_{t,+}(x)+g_{t,-}(x)-V_{t}(x)+l_{t}=-2 \tilde{\varphi}_{t}(x), & \text { for } x<a_{t},
\end{array}
$$

we see that the RH problem for $T$ is the following. 


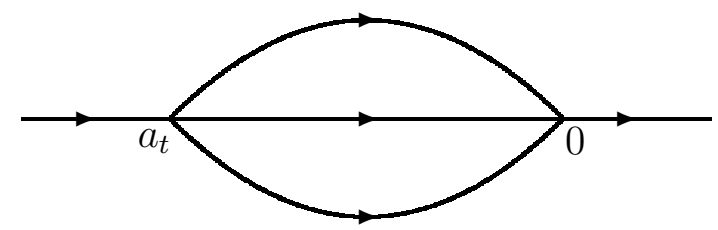

Figure 3: Opening of a lens around $\left[a_{t}, 0\right]$.

\section{Riemann-Hilbert problem for $T$}

- $T: \mathbb{C} \backslash \mathbb{R} \rightarrow \mathbb{C}^{2 \times 2}$ is analytic.

- $T_{+}(x)=T_{-}(x) v_{T}(x)$ for $x \in \mathbb{R}$, with

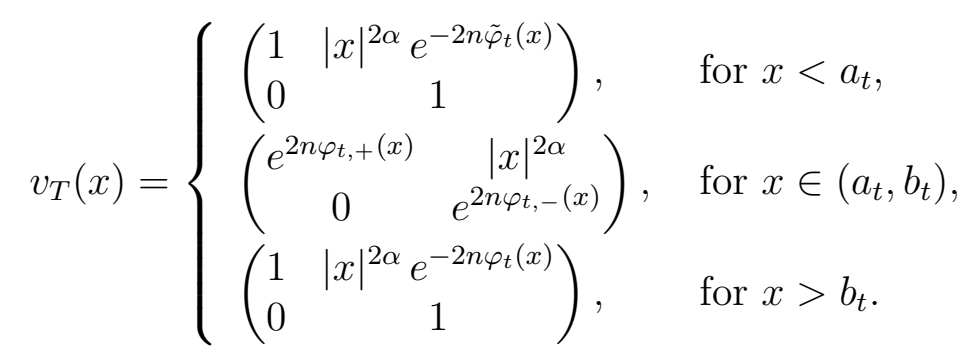

- $T(z)=I+O(1 / z)$ as $z \rightarrow \infty$.

- If $\alpha<0$, then $T(z)=O\left(\begin{array}{l}1|z|^{2 \alpha} \\ 1|z|^{2 \alpha}\end{array}\right)$ as $z \rightarrow 0$. If $\alpha \geq 0$, then $T(z)=O\left(\begin{array}{ll}1 & 1 \\ 1 & 1\end{array}\right)$ as $z \rightarrow 0$.

\subsubsection{Second transformation $T \mapsto S$}

The opening of lenses is based on the following factorization of $v_{T}$ on $\left(a_{t}, b_{t}\right)$ :

$$
\begin{aligned}
& v_{T}(x)=\left(\begin{array}{cc}
e^{2 n \varphi_{t,+}(x)} & |x|^{2 \alpha} \\
0 & e^{2 n \varphi_{t,-}(x)}
\end{array}\right) \\
& =\left(\begin{array}{cc}
1 & 0 \\
|x|^{-2 \alpha} e^{2 n \varphi_{t,-}(x)} & 1
\end{array}\right)\left(\begin{array}{cc}
0 & |x|^{2 \alpha} \\
-|x|^{-2 \alpha} & 0
\end{array}\right)\left(\begin{array}{cc}
1 & 0 \\
|x|^{-2 \alpha} e^{2 n \varphi_{t,+}(x)} & 1
\end{array}\right) .
\end{aligned}
$$

Introduce a lens around the segment $\left[a_{t}, 0\right]$ as in Figure 3 (recall that $a_{t}<0$ ). In the $\operatorname{disc} U^{(0)}$ around 0 we take the lens such that $z \mapsto \zeta=f_{t}(z)-f_{t}(0)$, see (2.51), maps the parts of the upper and lower lips of the lens that are in $U^{(0)}$ into the rays $\arg \zeta=2 \pi / 3$ and $\arg \zeta_{\tilde{f}}=-2 \pi / 3$, respectively. Similarly, in the disc $U^{(a)}$ we choose the lens so that $z \mapsto \zeta=\tilde{f}_{t}(z)$, see (2.54), maps the parts of the upper and lower lips of the lens that are in $U^{(a)}$ into the rays $\arg \zeta=\pi / 3$, and $\arg \zeta=-\pi / 3$, respectively. The remaining parts of the lips of the lens are arbitrary. However, they should be contained in the domain of analyticity of $V$, and we take them so that

$$
\operatorname{Re} \varphi_{t}(z)<-c<0
$$

for $z$ on the lips of the lens outside $U^{(0)}$ and $U^{(a)}$, with $c>0$ independent of $t$.

It is important to note that the lens is around $\left[a_{t}, 0\right]$, and not around $\left[a_{t}, b_{t}\right]$. 
Define $S$ by

$$
S(z)= \begin{cases}T(z), & \text { for } z \text { outside the lens } \\
T(z)\left(\begin{array}{cc}
1 & 0 \\
(-z)^{-2 \alpha} e^{2 n \varphi_{t}(z)} & 1
\end{array}\right)^{-1}, & \text { for } z \text { in the upper part of the lens } \\
T(z)\left(\begin{array}{cc}
1 & 0 \\
(-z)^{-2 \alpha} e^{2 n \varphi_{t}(z)} & 1
\end{array}\right), & \text { for } z \text { in the lower part of the lens. }\end{cases}
$$

Here the map $z \mapsto(-z)^{-2 \alpha}$ is defined with a cut along the positive real axis. Then, from (2.60) and the RH problem for $T$, we find that $S$ is the unique solution of the following $\mathrm{RH}$ problem.

\section{Riemann-Hilbert problem for $S$}

- $S: \mathbb{C} \backslash \Sigma_{S} \rightarrow \mathbb{C}^{2 \times 2}$ is analytic, where $\Sigma_{S}$ consists of the real line and the upper and lower lips of the lens, with orientation as in Figure 3 .

- $S_{+}(z)=S_{-}(z) v_{S}(z)$ for $z \in \Sigma_{S}$, where $v_{S}$ is given as follows. For $t<1$, so that $b_{t}<0$, we have

$$
v_{S}(z)= \begin{cases}\left(\begin{array}{cc}
1 & |z|^{2 \alpha} e^{-2 n \tilde{\varphi}_{t}(z)} \\
0 & 1
\end{array}\right), & \text { for } z \in\left(-\infty, a_{t}\right) \\
\left(\begin{array}{cc}
0 & |z|^{2 \alpha} \\
-|z|^{-2 \alpha} & 0
\end{array}\right), & \text { for } z \in\left(a_{t}, b_{t}\right) \\
\left(\begin{array}{cc}
0 & |z|^{2 \alpha} e^{-2 n \varphi_{t}(z)} \\
-|z|^{-2 \alpha} e^{2 n \varphi_{t}(z)} & 0
\end{array}\right), & \text { for } z \in\left(b_{t}, 0\right), \\
\left(\begin{array}{cc}
1 & |z|^{2 \alpha} e^{-2 n \varphi_{t}(z)} \\
0 & 1
\end{array}\right), & \text { for } z \in(0, \infty), \\
\left(\begin{array}{cc}
1 & 0 \\
(-z)^{-2 \alpha} e^{2 n \varphi_{t}(z)} & 1
\end{array}\right), & \text { for } z \text { on both lips of the lens, }\end{cases}
$$

while, for $t \geq 1$, so that $b_{t} \geq 0$, we have

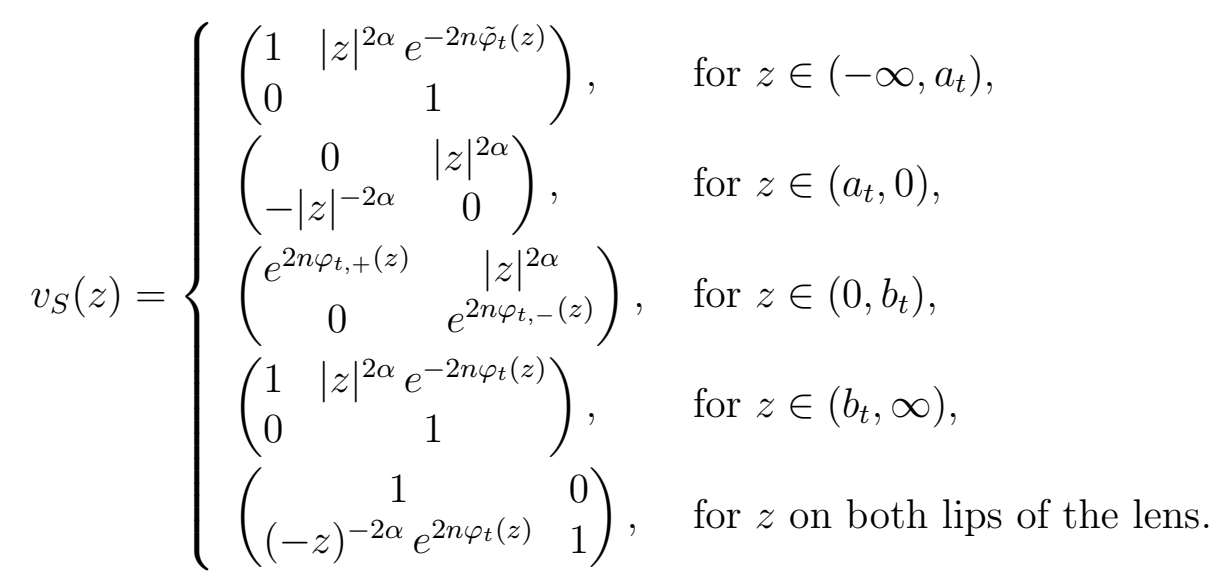

- $S(z)=I+O(1 / z)$ as $z \rightarrow \infty$.

- If $\alpha<0$, then $S(z)=O\left(\begin{array}{l}1|z|^{2 \alpha} \\ 1|z|^{2 \alpha}\end{array}\right)$ as $z \rightarrow 0$. If $\alpha \geq 0$, then $S(z)=O\left(\begin{array}{ll}1 & 1 \\ 1 & 1\end{array}\right)$ as $z \rightarrow 0$

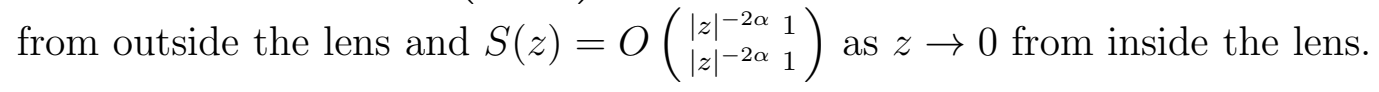


The next step is to approximate $S$ by a parametrix $P$, consisting of three parts $P^{(\infty)}$, $P^{(a)}$, and $P^{(0)}$ :

$$
P(z)= \begin{cases}P^{(0)}(z), & \text { for } z \in U^{(0)} \backslash \Sigma_{S} \\ P^{(a)}(z), & \text { for } z \in U^{(a)} \backslash \Sigma_{S} \\ P^{(\infty)}(z), & \text { for } z \in \mathbb{C} \backslash\left(\overline{U^{(0)} \cup U^{(a)} \cup\left(a_{t}, 0\right)}\right)\end{cases}
$$

where $U^{(a)}$ and $U^{(0)}$ are small discs centered at $a$ and 0 , respectively, that have been introduced before. The parametrices $P^{(\infty)}, P^{(a)}$ and $P^{(0)}$ are constructed below.

\subsubsection{The parametrix $P^{(\infty)}$}

The parametrix $P^{(\infty)}$ is a solution of the following $\mathrm{RH}$ problem.

Riemann-Hilbert problem for $P^{(\infty)}$

- $P^{(\infty)}: \mathbb{C} \backslash\left[a_{t}, 0\right] \rightarrow \mathbb{C}^{2 \times 2}$ is analytic.

- $P_{+}^{(\infty)}(x)=P_{-}^{(\infty)}(x)\left(\begin{array}{cc}0 & |x|^{2 \alpha} \\ -|x|^{-2 \alpha} & 0\end{array}\right)$ for $x \in\left(a_{t}, 0\right)$, oriented from left to right.

- $P^{(\infty)}(z)=I+O(1 / z)$ as $z \rightarrow \infty$.

The RH problem for $P^{(\infty)}$ can be explicitly solved as in [9]. Take

$$
D(z)=z^{\alpha} \phi\left(\frac{2 z-a_{t}}{-a_{t}}\right)^{-\alpha}, \quad \text { for } z \in \mathbb{C} \backslash\left[a_{t}, 0\right]
$$

where $\phi(z)=z+(z-1)^{1 / 2}(z+1)^{1 / 2}$ is the conformal map from $\mathbb{C} \backslash[-1,1]$ onto the exterior of the unit circle. Then $D_{+}(x) D_{-}(x)=|x|^{2 \alpha}$ for $x \in\left(a_{t}, 0\right)$. It follows that $D(\infty)^{-\sigma_{3}} P^{(\infty)}(z) D(z)^{\sigma_{3}}$ satisfies the normalized RH problem with jump matrix $\left(\begin{array}{rr}0 & 1 \\ -1 & 0\end{array}\right)$ on $\left(a_{t}, 0\right)$ (oriented from left to right), whose solution is well-known, see e.g. [11, 14], and it leads to

$$
P^{(\infty)}(z)=D(\infty)^{\sigma_{3}}\left(\begin{array}{cc}
\frac{1}{2}\left(\beta_{t}(z)+\beta_{t}(z)^{-1}\right) & \frac{1}{2 i}\left(\beta_{t}(z)-\beta_{t}(z)^{-1}\right) \\
-\frac{1}{2 i}\left(\beta_{t}(z)-\beta_{t}(z)^{-1}\right) & \frac{1}{2}\left(\beta_{t}(z)+\beta_{t}(z)^{-1}\right)
\end{array}\right) D(z)^{-\sigma_{3}}
$$

for $z \in \mathbb{C} \backslash\left[a_{t}, 0\right]$, where

$$
\beta_{t}(z)=\left(\frac{z}{z-a_{t}}\right)^{1 / 4}, \quad \text { for } z \in \mathbb{C} \backslash\left[a_{t}, 0\right]
$$

\subsubsection{The parametrix $P^{(a)}$}

The parametrix $P^{(a)}$ is defined in the $\operatorname{disc} U^{(a)}$ around $a$, where $P^{(a)}$ satisfies the following RH problem. 
Riemann-Hilbert problem for $P^{(a)}$

- $P^{(a)}: U^{(a)} \backslash \Sigma_{S} \rightarrow \mathbb{C}^{2 \times 2}$ is analytic.

- $P_{+}^{(a)}(z)=P_{-}^{(a)}(z) v_{S}(z)$ for $z \in U^{(a)} \cap \Sigma_{S}$.

- $P^{(a)}(z)\left(P^{(\infty)}(z)\right)^{-1}=I+O\left(n^{-1}\right)$, as $n \rightarrow \infty$, uniformly for $z \in \partial U^{(a)} \backslash \Sigma_{S}$.

We seek $P^{(a)}$ in the form

$$
P^{(a)}(z)=\widehat{P}^{(a)}(z) e^{n \tilde{\varphi}_{t}(z) \sigma_{3}}(-z)^{-\alpha \sigma_{3}}, \quad \text { for } z \in U^{(a)} \backslash \Sigma_{S},
$$

where $(-z)^{-\alpha}$ is defined with a branch cut along $[0, \infty)$. Then $\widehat{P}^{(a)}$ satisfies a RH problem with constant jumps and can be constructed in terms of the Airy function in a standard way; for more details see the presentation in [11].

\subsubsection{The parametrix $P^{(0)}$}

The parametrix $P^{(0)}$, defined in the disk $U^{(0)}$ around 0 , should satisfy the following $\mathrm{RH}$ problem.

Riemann-Hilbert problem for $P^{(0)}$

- $P^{(0)}: \overline{U^{(0)}} \backslash \Sigma_{S} \rightarrow \mathbb{C}^{2 \times 2}$ is continuous and analytic on $U^{(0)} \backslash \Sigma_{S}$.

- $P_{+}^{(0)}(z)=P_{-}^{(0)}(z) v_{S}(z)$ for $z \in \Sigma_{S} \cap U^{(0)}$ (with the same orientation as $\Sigma_{S}$ ).

- $P^{(0)}(z)\left(P^{(\infty)}(z)\right)^{-1}=I+O\left(n^{-1 / 3}\right)$, as $n \rightarrow \infty, t \rightarrow 1$ such that $n^{2 / 3}(t-1)=O(1)$, uniformly for $z \in \partial U^{(0)} \backslash \Sigma_{S}$.

- $P^{(0)}$ has the same behavior near 0 as $S$ has (see the $\mathrm{RH}$ problem for $S$ ).

A parametrix $P^{(0)}$ with these properties can be constructed using a solution $\Psi_{\alpha}$ of the model RH problem of Subsection 1.2. The construction is done in three steps.

Step 1: Transformation to constant jumps. We seek $P^{(0)}$ in the form

$$
P^{(0)}(z)=\widehat{P}^{(0)}(z) e^{n \varphi_{t}(z) \sigma_{3}} z^{-\alpha \sigma_{3}}, \quad \text { for } z \in \overline{U^{(0)}} \backslash \Sigma_{S},
$$

where as usual $z^{-\alpha}$ denotes the principal branch. It then follows from the RH problem for $P^{(0)}$ that $\widehat{P}^{(0)}$ should satisfy the following $\mathrm{RH}$ problem.

Riemann-Hilbert problem for $\widehat{P}^{(0)}$

- $\widehat{P}^{(0)}: \overline{U^{(0)}} \backslash \Sigma_{S} \rightarrow \mathbb{C}^{2 \times 2}$ is continuous and analytic on $U^{(0)} \backslash \Sigma_{S}$. 
- For $z \in \Sigma_{S} \cap U^{(0)}$, we have

$$
\widehat{P}_{+}^{(0)}(z)=\widehat{P}_{-}^{(0)}(z) \times \begin{cases}\left(\begin{array}{cc}
1 & 1 \\
0 & 1
\end{array}\right), & \text { for } z \in(0, \infty) \cap U^{(0)} \\
\left(\begin{array}{cc}
1 & 0 \\
e^{2 \alpha \pi i} & 1
\end{array}\right), & \text { for } z \text { in } U^{(0)} \text { on the upper lip of the lens, } \\
\left(\begin{array}{cc}
0 & 1 \\
-1 & 0
\end{array}\right), & \text { for } z \in(-\infty, 0) \cap U^{(0)} \\
\left(\begin{array}{cc}
1 & 0 \\
e^{-2 \alpha \pi i} & 1
\end{array}\right), & \text { for } z \text { in } U^{(0)} \text { on the lower lip of the lens. }\end{cases}
$$

uniformly for $z \in \partial U^{(0)} \backslash \Sigma_{S}$.

- If $\alpha<0$, then

$$
\widehat{P}^{(0)}(z)=O\left(\begin{array}{ll}
|z|^{\alpha} & |z|^{\alpha} \\
|z|^{\alpha} & |z|^{\alpha}
\end{array}\right) \quad \text { as } z \rightarrow 0
$$

while if $\alpha \geq 0$ we have that

$$
\begin{aligned}
& \widehat{P}^{(0)}(z)=O\left(\begin{array}{ll}
|z|^{\alpha} & |z|^{-\alpha} \\
|z|^{\alpha} & |z|^{-\alpha}
\end{array}\right) \quad \text { as } z \rightarrow 0 \text { from outside the lens, and } \\
& \widehat{P}^{(0)}(z)=O\left(\begin{array}{ll}
|z|^{-\alpha} & |z|^{-\alpha} \\
|z|^{-\alpha} & |z|^{-\alpha}
\end{array}\right) \quad \text { as } z \rightarrow 0 \text { from inside the lens. }
\end{aligned}
$$

Note that the jump matrices of $\widehat{P}^{(0)}$ do not depend on $t$.

The reader may note the similarities between the above $\mathrm{RH}$ problem for $\widehat{P}^{(0)}$ and the RH problem for $\Psi_{\alpha}$ from Subsection 1.2. In the next step we show how we can use $\Psi_{\alpha}$ to construct a solution of the RH problem for $\widehat{P}^{(0)}$.

Step 2: The construction of $\widehat{P}^{(0)}$ in terms of $\Psi_{\alpha}$. Recall that $\Sigma_{S}$ in $U^{(0)}$ was taken such that $z \mapsto f_{t}(z)-f_{t}(0)$ maps $\Sigma_{S} \cap U^{(0)}$ onto a subset of $\Sigma$, where $\Sigma$ is the contour in the RH problem for $\Psi_{\alpha}$, see Subsection 1.2,

We choose any solution $\Psi_{\alpha}$ of the model $\mathrm{RH}$ problem and we define $\widehat{P}^{(0)}$ by

$$
\widehat{P}^{(0)}(z)=E(z) \Psi_{\alpha}\left(n^{\frac{2}{3}}\left(f_{t}(z)-f_{t}(0)\right) ; n^{\frac{2}{3}} f_{t}(0)\right), \quad \text { for } z \in \overline{U^{(0)}} \backslash \Sigma_{S}
$$

where $E=E_{n, N}$ is analytic in $U^{(0)}$. Taking $P^{(0)}$ as in (2.65) with $\widehat{P}^{(0)}$ as in (2.66) we find that all the conditions of the $\mathrm{RH}$ problem for $P^{(0)}$ are satisfied, except for the matching condition

$$
P^{(0)}(z)\left(P^{(\infty)}(z)\right)^{-1}=I+O\left(n^{-1 / 3}\right)
$$

as $n \rightarrow \infty, t \rightarrow 1$ such that $n^{2 / 3}(t-1)=O(1)$. 
Step 3: Matching condition. To be able to satisfy (2.67) we have to take $E$ in the following way

$$
E(z)=P^{(\infty)}(z) z^{\alpha \sigma_{3}} \frac{1}{\sqrt{2}}\left(\begin{array}{cc}
1 & -i \\
-i & 1
\end{array}\right)\left(n^{\frac{2}{3}}\left(f_{t}(z)-f_{t}(0)\right)\right)^{\sigma_{3} / 4}, \quad \text { for } z \in U^{(0)} \backslash\left[a_{t}, 0\right]
$$

where both branches are taken as principal. Clearly then $E$ is analytic in $U^{(0)} \backslash\left[a_{t}, 0\right]$. It turns out that $E$ has analytic continuation to $U^{(0)}$. This follows by direct calculation, but it relies on the fact that we chose $\left[a_{t}, 0\right]$ as the jump contour for $P^{(\infty)}$.

With the choice (2.68) for $E$, we now show that (2.67) is satisfied as well. By (2.65), (2.66), we have for $z \in \partial U^{(0)} \backslash \Sigma_{S}$,

$$
P^{(0)}(z)=E(z) \Psi_{\alpha}\left(n^{\frac{2}{3}}\left(f_{t}(z)-f_{t}(0)\right) ; n^{\frac{2}{3}} f_{t}(0)\right) e^{n \varphi_{t} \sigma_{3}} z^{-\alpha \sigma_{3}}
$$

and we are interested in the behavior as $n \rightarrow \infty, t \rightarrow 1$ such that $n^{2 / 3}(t-1)=O(1)$.

We show first that $n^{2 / 3} f_{t}(0)$ remains bounded.

Lemma 2.7 Suppose $n \rightarrow \infty, t \rightarrow 1$ such that $n^{2 / 3}(t-1)=O(1)$. Then $n^{2 / 3} f_{t}(0)$ remains bounded. More precisely, if $n^{2 / 3}(t-1) \rightarrow L \in \mathbb{R}$, then

$$
n^{2 / 3} f_{t}(0) \rightarrow-c_{2, V} L=s,
$$

where

$$
c_{2, V}=\left.\left(c_{1, V}\right)^{2 / 3} \frac{d b_{t}}{d t}\right|_{t=1}
$$

and $c_{1, V}$ is the constant in (1.8).

Proof. It follows from (2.51), that

$$
\begin{aligned}
f_{t}(0) & =\left(\frac{1}{2} \sqrt{-a_{t}} h_{t}\left(b_{t}\right)\right)^{2 / 3}\left(-b_{t}\right) \chi_{t}^{2 / 3}(0) \\
& =-\left.\left(\frac{1}{2} \sqrt{-a} h_{1}(0)\right)^{2 / 3}(t-1) \frac{d b_{t}}{d t}\right|_{t=1}+O\left((t-1)^{2}\right) \quad \text { as } t \rightarrow 1 .
\end{aligned}
$$

By (1.8) and (2.46), we have

$$
c_{1, V}=\frac{1}{2} \sqrt{-a} h_{1}(0),
$$

so that (2.69)-(2.70) indeed follows if $n^{2 / 3}(t-1) \rightarrow L$.

If we use the formula (2.47) for the $t$-derivative of $b_{t}$ at $t=1$, then we find from (2.70) that

$$
c_{2, V}=2(-a)^{-1 / 2} c_{1, V}^{-1 / 3}
$$

Now we continue with the proof of (2.67). 
Lemma 2.8 Suppose that $n \rightarrow \infty, t \rightarrow 1$ such that $n^{2 / 3}(t-1)=O(1)$. Then (2.67) holds.

Proof. In the proof all $O$-terms are for $n \rightarrow \infty, t \rightarrow 1$ such that $n^{2 / 3}(t-1)$ is bounded.

By Lemma 2.7 the values $n^{2 / 3} f_{t}(0)$ remain bounded. Since the asymptotic condition (c) in the RH problem for $\Psi_{\alpha}$ is valid uniformly for $s$ in bounded subsets of $\mathbb{R}$, we find by (2.65), (2.66), and (2.68)

$$
\begin{aligned}
P^{(0)}(z)= & E(z)\left(n^{\frac{2}{3}}\left(f_{t}(z)-f_{t}(0)\right)\right)^{-\sigma_{3} / 4} \frac{1}{\sqrt{2}}\left(\begin{array}{cc}
1 & i \\
i & 1
\end{array}\right)\left(I+O\left(n^{-1 / 3}\right)\right) \\
& \quad \times \exp \left(-\theta\left(n^{2 / 3}\left(f_{t}(z)-f_{t}(0)\right) ; n^{2 / 3} f_{t}(0)\right) \sigma_{3}\right) e^{n \varphi_{t} \sigma_{3}} z^{-\alpha \sigma_{3}} \\
= & P^{(\infty)}(z)\left(I+O\left(n^{-1 / 3}\right)\right) \exp \left(-\left(\theta\left(n^{2 / 3}\left(f_{t}(z)-f_{t}(0)\right) ; n^{2 / 3} f_{t}(0)\right)-n \varphi_{t}\right) \sigma_{3}\right)
\end{aligned}
$$

uniformly for $z \in \partial U^{(0)}$. As before we denote $\theta(\zeta ; s)=\frac{2}{3} \zeta^{3 / 2}+s \zeta^{1 / 2}$.

The next step is to evaluate the expression in the exponential factor. We have

$$
\begin{aligned}
& \theta\left(n^{2 / 3}\left(f_{t}(z)-f_{t}(0)\right) ; n^{2 / 3} f_{t}(0)\right)-n \varphi_{t} \\
& =\frac{2}{3} n\left[\left(f_{t}(z)-f_{t}(0)\right)^{3 / 2}-\left(f_{t}(z)\right)^{3 / 2}\right]+n f_{t}(0)\left(f_{t}(z)-f_{t}(0)\right)^{1 / 2} .
\end{aligned}
$$

We will show that this is $O\left(n^{-1 / 3}\right)$ uniformly for $z \in \partial U^{(0)}$. To that end, it is enough to show that

$$
\begin{aligned}
F(t, z) & :=\left(f_{t}(z)-f_{t}(0)\right)^{3 / 2}-\left(f_{t}(z)\right)^{3 / 2}+\frac{3}{2} f_{t}(0)\left(f_{t}(z)-f_{t}(0)\right)^{1 / 2} \\
& =O\left((t-1)^{2}\right) \quad \text { as } t \rightarrow 1,
\end{aligned}
$$

uniformly for $z \in \partial U^{(0)}$.

By (2.53), we have

$$
F(1, z)=0 \text {. }
$$

Moreover,

$$
\begin{aligned}
\frac{\partial}{\partial t} F(t, z)=\frac{3}{2} & \left(f_{t}(z)-f_{t}(0)\right)^{\frac{1}{2}} \frac{\partial}{\partial t}\left(f_{t}(z)-f_{t}(0)\right)-\frac{3}{2}\left(f_{t}(z)\right)^{\frac{1}{2}} \frac{\partial}{\partial t} f_{t}(z) \\
& +\frac{3}{2}\left(\frac{d}{d t} f_{t}(0)\right)\left(f_{t}(z)-f_{t}(0)\right)^{\frac{1}{2}} \\
& +\frac{3}{2} f_{t}(0) \frac{1}{2}\left(f_{t}(z)-f_{t}(0)\right)^{-\frac{1}{2}} \frac{\partial}{\partial t}\left(f_{t}(z)-f_{t}(0)\right) .
\end{aligned}
$$

Let $t=1$ and again use (2.53) and (2.51). Due to cancellations one finds

$$
\frac{\partial}{\partial t} F(1, z)=0
$$

Since, in addition, $F(t, z)$ is analytic in both variables and bounded with respect to $z$ in $\partial U^{(0)}$, it follows from a Taylor expansion that $F(t, z)=O\left((t-1)^{2}\right)$, as claimed in (2.74). Thus

$$
\theta\left(n^{2 / 3}\left(f_{t}(z)-f_{t}(0)\right) ; n^{2 / 3} f_{t}(0)\right)-n \varphi_{t}=O\left(n^{-1 / 3}\right),
$$




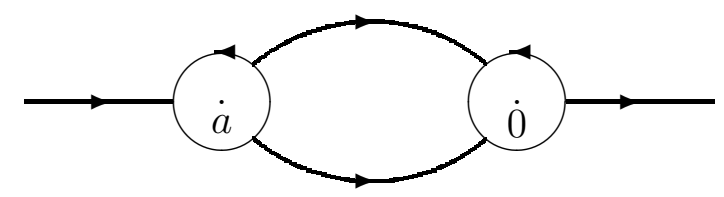

Figure 4: Contour for the RH problem for $R$.

so that (2.73) leads to

$$
P^{(0)}(z)=P^{(\infty)}(z)\left(I+O\left(n^{-1 / 3}\right)\right)
$$

uniformly for $z \in \partial U^{(0)}$. Then (2.67) follows since $P^{(\infty)}(z)$ and its inverse are bounded in $n$ and $t$, uniformly for $z \in \partial U^{(0)}$.

This completes the construction of the parametrix $P^{(0)}$.

Remark 2.9 The local parametrix $P^{(0)}$ is constructed with the help of a solution $\Psi_{\alpha}$ of the model RH problem. Since the solution $\Psi_{\alpha}$ is not unique (see Proposition 2.1), the local parametrix is not unique. In what follows we can take any $P^{(0)}$ and it will not affect the final results (Theorems 1.1 and 1.2).

\subsubsection{Third transformation $S \mapsto R$}

Having $P^{(\infty)}, P^{(a)}$, and $P^{(0)}$, we take $P$ as in (2.61), and then we define

$$
R(z)=S(z) P^{-1}(z), \quad \text { for } z \in \mathbb{C} \backslash\left(\partial U^{(0)} \cup \partial U^{(a)} \cup \Sigma_{S}\right) .
$$

Since $S$ and $P$ have the same jump matrices on $U^{(0)} \cap \Sigma_{S}, U^{(a)} \cap \Sigma_{S}$ and $(a, 0) \backslash\left(U^{(0)} \cup U^{(a)}\right)$, we have that $R$ is analytic across these contours. What remains are jumps for $R$ on the contour $\Sigma_{R}$ shown in Figure 4 with orientation that is also shown in the figure. Then, $R$ satisfies the following $\mathrm{RH}$ problem.

\section{Riemann-Hilbert problem for $R$}

- $R: \mathbb{C} \backslash \Sigma_{R} \rightarrow \mathbb{C}^{2 \times 2}$ is analytic.

- $R_{+}(z)=R_{-}(z) v_{R}(z)$ for $z \in \Sigma_{R}$, where

$$
v_{R}= \begin{cases}P^{(\infty)}\left(P^{(0)}\right)^{-1}, & \text { on } \partial U^{(0)} \\ P^{(\infty)}\left(P^{(a)}\right)^{-1}, & \text { on } \partial U^{(a)} \\ P^{(\infty)} v_{S}\left(P^{(\infty)}\right)^{-1}, & \text { on } \Sigma_{R} \backslash\left(\partial U^{(0)} \cup \partial U^{(a)}\right) .\end{cases}
$$

- $R(z)=I+O(1 / z)$ as $z \rightarrow \infty$.

Now let $n \rightarrow \infty, t \rightarrow 1$ such that $n^{2 / 3}(t-1)=O(1)$. It then follows from the construction of the parametrices (see in particular the $\mathrm{RH}$ problems for $P^{(0)}$ and $P^{(a)}$ ) that

$$
v_{R}= \begin{cases}I+O\left(n^{-1 / 3}\right), & \text { on } \partial U^{(0)}, \\ I+O\left(n^{-1}\right), & \text { on } \partial U^{(a)} .\end{cases}
$$


Furthermore, by regularity of the eigenvalue density, there is a constant $c>0$ such that

$$
\begin{array}{ll}
\operatorname{Re} \varphi_{t}(z)>c>0, & \text { for } z \in \Sigma_{R} \cap(0, \infty), \\
\operatorname{Re} \tilde{\varphi}_{t}(z)>c>0, & \text { for } z \in \Sigma_{R} \cap(-\infty, a), \\
\operatorname{Re} \varphi_{t}(z)<-c<0, & \text { for } z \in \Sigma_{R} \backslash\left(\partial U^{(0)} \cup \partial U^{(a)} \cup \mathbb{R}\right) .
\end{array}
$$

This implies (see the RH problem for $S$ ) that $v_{S}=I+O\left(e^{-c n}\right)$ uniformly on $\Sigma_{R} \backslash\left(\partial U^{(0)} \cup\right.$ $\left.\partial U^{(a)}\right)$, so that by (2.78)

$$
v_{R}=I+O\left(e^{-2 c n}\right) \quad \text { on } \Sigma_{R} \backslash\left(\partial U^{(0)} \cup \partial U^{(a)}\right) .
$$

The $O$-terms in (2.79) and (2.80) are uniform on the indicated contours. In addition, it follows from (2.58), (2.59), (2.55), and the growth condition (1.2) on $V$ that for any $C>0$ there exists $r=r(C)>1$ such that $\varphi_{t}(x) \geq C \log x$ for $x \geq r$, and $\tilde{\varphi}_{t}(x) \geq C \log |x|$ for $x \leq-r$. Combined with (2.80) this implies that

$$
\left.\left\|v_{R}-I\right\|\right|_{L^{2}\left(\Sigma_{R} \backslash\left(\partial U^{(0)} \cup \partial U^{(a)}\right)\right)}=O\left(e^{-2 c n}\right), \quad \text { as } n \rightarrow \infty .
$$

Thus, by (2.79) -(2.81), as $n \rightarrow \infty$ and $t \rightarrow 1$ such that $n^{2 / 3}(t-1)=O(1)$, the jump matrix for $R$ is close to $I$ in both $L^{2}$ and $L^{\infty}$ norm on $\Sigma_{R}$, indeed

$$
\left\|v_{R}-I\right\|_{L^{2}\left(\Sigma_{R}\right) \cap L^{\infty}\left(\Sigma_{R}\right)}=O\left(n^{-1 / 3}\right) .
$$

Standard estimates using $L^{2}$-boundedness of the operators $C_{ \pm}$on $L^{2}\left(\Sigma_{R}\right)$ together with the correspondence between $\mathrm{RH}$ problems and singular integral equations now imply that

$$
R(z)=I+O\left(n^{-1 / 3}\right), \quad \text { uniformly for } z \in \mathbb{C} \backslash \Sigma_{R},
$$

as $n \rightarrow \infty, t \rightarrow 1$ such that $n^{2 / 3}(t-1)=O(1)$. To get the uniform bound (2.83) up to the contour one needs a contour deformation argument. Again, see the presentation in [11] for more details.

This completes the steepest descent analysis of the $\mathrm{RH}$ problem for $Y$.

\subsection{Completion of the proofs of Theorem 1.1 and 1.2}

Having completed the steepest descent analysis we are now ready for the proofs of Theorem 1.1 and 1.2. We start by rewriting the kernel (2.3) for $x, y \in U^{(0)} \cap \mathbb{R}$ according to the transformations $Y \mapsto T \mapsto S \mapsto R$ that we did in the steepest descent analysis. To state the result it is convenient to introduce $B=B_{n, N}$ as

$$
B(z)=R(z) E(z), \quad \text { for } z \in U^{(0)},
$$

where $E$ and $R$ are defined in (2.68) and (2.77). We also define for $x, s \in \mathbb{R}$ the column vector

$$
\vec{\psi}_{\alpha}(x ; s)=\left(\begin{array}{l}
\psi_{1}(x ; s) \\
\psi_{2}(x ; s)
\end{array}\right)= \begin{cases}\Psi_{\alpha,+}(x ; s)\left(\begin{array}{l}
1 \\
0
\end{array}\right), & \text { for } x>0 \\
\Psi_{\alpha,+}(x ; s) e^{-\alpha \pi i \sigma_{3}}\left(\begin{array}{l}
1 \\
1
\end{array}\right), & \text { for } x<0\end{cases}
$$

cf. (1.12). We then have the following result. 
Lemma 2.10 Let $x, y \in U^{(0)} \cap \mathbb{R}$. Then,

$$
\begin{aligned}
K_{n, N}(x, y)= & \frac{1}{2 \pi i(x-y)}\left(\vec{\psi}_{\alpha}\left(n^{\frac{2}{3}}\left(f_{t}(y)-f_{t}(0)\right) ; n^{\frac{2}{3}} f_{t}(0)\right)\right)^{T}\left(\begin{array}{cc}
0 & 1 \\
-1 & 0
\end{array}\right) \\
& \times B^{-1}(y) B(x) \vec{\psi}_{\alpha}\left(n^{\frac{2}{3}}\left(f_{t}(x)-f_{t}(0)\right) ; n^{\frac{2}{3}} f_{t}(0)\right) .
\end{aligned}
$$

Proof. We start from the formula (2.3) for the eigenvalue correlation kernel. Using (2.56) we obtain, for any $x, y \in \mathbb{R}$,

$$
\begin{aligned}
K_{n, N}(x, y)=|x|^{\alpha} e^{\frac{1}{2} n\left(2 g_{t,+}(x)-V_{t}(x)+l_{t}\right)}|y|^{\alpha} e^{\frac{1}{2} n\left(2 g_{t,+}(y)-V_{t}(y)+l_{t}\right)} \\
\times \frac{1}{2 \pi i(x-y)}\left(\begin{array}{ll}
0 & 1
\end{array}\right) T_{+}^{-1}(y) T_{+}(x)\left(\begin{array}{l}
1 \\
0
\end{array}\right) .
\end{aligned}
$$

Using (2.58) and the fact that $g_{t,+}=g_{t,-}$ on $\left(b_{t}, \infty\right)$, it follows that $2 g_{t}-V_{t}+l_{t}=-2 \varphi_{t}$ on $\left(b_{t}, \infty\right)$. Then, by analytic continuation, $2 g_{t,+}-V_{t}+l_{t}=-2 \varphi_{t,+}$ on all of $\mathbb{R}$. Therefore we can rewrite (2.87) as

$$
K_{n, N}(x, y)=|x|^{\alpha} e^{-n \varphi_{t,+}(x)}|y|^{\alpha} e^{-n \varphi_{t,+}(y)} \frac{1}{2 \pi i(x-y)}\left(\begin{array}{ll}
0 & 1
\end{array}\right) T_{+}^{-1}(y) T_{+}(x)\left(\begin{array}{l}
1 \\
0
\end{array}\right) .
$$

Now we analyze the effect of the transformations $T \mapsto S \mapsto R$ on the expression $|x|^{\alpha} e^{-n \varphi_{t,+}(x)} T_{+}(x)\left(\begin{array}{l}1 \\ 0\end{array}\right)$ in case $x \in U^{(0)} \cap \mathbb{R}$. The result is that for $x \in U^{(0)} \cap \mathbb{R}$,

$$
|x|^{\alpha} e^{-n \varphi_{t,+}(x)} T_{+}(x)\left(\begin{array}{l}
1 \\
0
\end{array}\right)=B(x) \Psi_{\alpha,+}\left(n^{\frac{2}{3}}\left(f_{t}(x)-f_{t}(0)\right) ; n^{\frac{2}{3}} f_{t}(0)\right)\left(\begin{array}{l}
1 \\
0
\end{array}\right)
$$

in case $x>0$, and

$$
|x|^{\alpha} e^{-n \varphi_{t,+}(x)} T_{+}(x)\left(\begin{array}{l}
1 \\
0
\end{array}\right)=B(x) \Psi_{\alpha,+}\left(n^{\frac{2}{3}}\left(f_{t}(x)-f_{t}(0)\right) ; n^{\frac{2}{3}} f_{t}(0)\right) e^{-\alpha \pi i \sigma_{3}}\left(\begin{array}{l}
1 \\
1
\end{array}\right)
$$

in case $x<0$. Since the calculations for (2.89) are easier, we will only show how to obtain (2.90). If $x \in U^{(0)} \cap \mathbb{R}$ and $x<0$, then it follows from (2.60) that

$$
\begin{aligned}
& |x|^{\alpha} e^{-n \varphi_{t,+}(x)} T_{+}(x)\left(\begin{array}{l}
1 \\
0
\end{array}\right)=|x|^{\alpha} e^{-n \varphi_{t,+}(x)} S_{+}(x)\left(\begin{array}{c}
1 \\
|x|^{-2 \alpha} e^{2 n \varphi_{t,+}(x)}
\end{array}\right) \\
& =S_{+}(x)\left(|x|^{\alpha} e^{-n \varphi_{t,+}(x)}\right)^{\sigma_{3}}\left(\begin{array}{l}
1 \\
1
\end{array}\right) .
\end{aligned}
$$

From (2.77), (2.61), (2.65), (2.68), and (2.84), we find that

$$
S_{+}(x)=B(x) \Psi_{\alpha,+}\left(n^{\frac{2}{3}}\left(f_{t}(x)-f_{t}(0)\right) ; n^{\frac{2}{3}} f_{t}(0)\right)\left(e^{n \varphi_{t}(x)} x^{-\alpha}\right)_{+}^{\sigma_{3}} .
$$

Inserting this into (2.91) and noting that $x_{+}^{-\alpha}|x|^{\alpha}=e^{-\alpha \pi i}$ we indeed obtain (2.90).

In a similar way, we find for $y \in U^{(0)} \cap \mathbb{R}$,

$$
|y|^{\alpha} e^{-n \varphi_{t,+}(y)}\left(\begin{array}{ll}
0 & 1
\end{array}\right) T_{+}^{-1}(y)=\left(\begin{array}{ll}
0 & 1
\end{array}\right) \Psi_{\alpha,+}^{-1}\left(n^{\frac{2}{3}}\left(f_{t}(y)-f_{t}(0)\right) ; n^{\frac{2}{3}} f_{t}(0)\right) B^{-1}(y),
$$


in case $y>0$, and

$$
|y|^{\alpha} e^{-n \varphi_{t,+}(y)}\left(\begin{array}{ll}
0 & 1
\end{array}\right) T_{+}^{-1}(y)=\left(\begin{array}{ll}
-1 & 1
\end{array}\right) e^{\alpha \pi i \sigma_{3}} \Psi_{\alpha,+}^{-1}\left(n^{\frac{2}{3}}\left(f_{t}(y)-f_{t}(0)\right) ; n^{\frac{2}{3}} f_{t}(0)\right) B^{-1}(y),
$$

in case $y<0$. To rewrite (2.92) and (2.93) we use the following fact, which is easy to check. If $A$ is an invertible $2 \times 2$ matrix having determinant 1 , then

$$
A^{-1}=\left(\begin{array}{cc}
0 & -1 \\
1 & 0
\end{array}\right) A^{T}\left(\begin{array}{cc}
0 & 1 \\
-1 & 0
\end{array}\right) .
$$

If we apply (2.94) to $\Psi_{\alpha,+}$ in (2.92) and (2.93), then we get

$$
\begin{aligned}
|y|^{\alpha} e^{-n \varphi_{t,+}(y)}\left(\begin{array}{ll}
0 & 1
\end{array}\right) T_{+}^{-1}(y)=\left(\begin{array}{ll}
1 & 0
\end{array}\right) \Psi_{\alpha,+}^{T}\left(n^{\frac{2}{3}}\left(f_{t}(y)-f_{t}(0)\right) ; n^{\frac{2}{3}} f_{t}(0)\right) \\
\times\left(\begin{array}{cc}
0 & 1 \\
-1 & 0
\end{array}\right) B^{-1}(y),
\end{aligned}
$$

in case $y>0$, and

$$
\begin{aligned}
|y|^{\alpha} e^{-n \varphi_{t,+}(y)}\left(\begin{array}{ll}
0 & 1
\end{array}\right) T_{+}^{-1}(y)=\left(\begin{array}{ll}
1 & 1
\end{array}\right) e^{-\alpha \pi i \sigma_{3}} \Psi_{\alpha,+}^{T}\left(n^{\frac{2}{3}}\left(f_{t}(y)-f_{t}(0)\right) ; n^{\frac{2}{3}} f_{t}(0)\right) \\
\times\left(\begin{array}{cc}
0 & 1 \\
-1 & 0
\end{array}\right) B^{-1}(y),
\end{aligned}
$$

in case $y<0$. Then (2.86) follows if we insert (2.89), (2.90), (2.95), and (2.96) into (2.88) and use the definition (2.85).

As in Theorem 1.1 we now fix $x, y \in \mathbb{R}$. We define

$$
x_{n}=\frac{x}{\left(c_{1} n\right)^{2 / 3}}, \quad \text { and } \quad y_{n}=\frac{y}{\left(c_{1} n\right)^{2 / 3}}
$$

where $c_{1}$ is the constant from (1.8).

In order to take the limit of $\left(c_{1} n\right)^{-2 / 3} K_{n, N}\left(x_{n}, y_{n}\right)$ we need one more lemma. Recall that $B=R E$ is defined in (2.84).

Lemma 2.11 Let $n \rightarrow \infty, t \rightarrow 1$ such that $n^{2 / 3}(t-1) \rightarrow$ L. Let $x, y \in \mathbb{R}$ and let $x_{n}$ and $y_{n}$ defined as in (2.97), Then the following hold.

(a) $n^{2 / 3} f_{t}(0) \rightarrow s$,

(b) $n^{2 / 3}\left(f_{t}\left(x_{n}\right)-f_{t}(0)\right) \rightarrow x$ and $n^{2 / 3}\left(f_{t}\left(y_{n}\right)-f_{t}(0)\right) \rightarrow y$,

(c) $B^{-1}\left(y_{n}\right) B\left(x_{n}\right)=I+O\left(\frac{x-y}{n^{1 / 3}}\right)$ where the implied constant in the O-term is uniform with respect to $x$ and $y$.

Proof. (a) This follows from Lemma 2.7.

(b) By (1.8) and (2.46) we have $c_{1}=\frac{1}{2} \sqrt{-a} h_{1}(0)$, so that $f_{1}^{\prime}(0)=c_{1}^{2 / 3}$ by (2.52). Taking note of the definitions (2.97), we then obtain part (b), since $f_{t} \rightarrow f_{1}$ uniformly in $U^{(0)}$. 
(c) We have

$$
\begin{aligned}
R^{-1}\left(y_{n}\right) R\left(x_{n}\right) & =I+R^{-1}\left(y_{n}\right)\left(R\left(x_{n}\right)-R\left(y_{n}\right)\right) \\
& =I+R^{-1}\left(y_{n}\right)\left(x_{n}-y_{n}\right) \int_{0}^{1} R^{\prime}\left(t x_{n}+(1-t) y_{n}\right) d t
\end{aligned}
$$

Recall that $R$ is analytic in $U^{(0)}$, and that $R(z)=I+O\left(n^{-1 / 3}\right)$ by (2.83), uniformly in $U^{(0)}$. Since $\operatorname{det} R \equiv 1$, we find that $R^{-1}\left(y_{n}\right)$ remains bounded as $n \rightarrow \infty$. It also follows from (2.83) and Cauchy's theorem, that $R^{\prime}(z)=O\left(n^{-1 / 3}\right)$ for $z$ in a neighborhood of the origin. By (2.98) we then obtain

$$
R^{-1}\left(y_{n}\right) R\left(x_{n}\right)=I+O\left(\frac{x-y}{n}\right) .
$$

Using analyticity of $E$ in a neighborhood of the origin with $E(z)=O\left(n^{1 / 6}\right)$, see (2.68), and the fact that $\operatorname{det} E \equiv 1$, we obtain in the same way

$$
E^{-1}\left(y_{n}\right) E\left(x_{n}\right)=I+O\left(\frac{x-y}{n^{1 / 3}}\right)
$$

The implied constants in (2.99) and (2.100) are independent of $x$ and $y$.

Using (2.99), (2.100), and the fact that $E\left(x_{n}\right)=O\left(n^{1 / 6}\right)$ and $E^{-1}\left(y_{n}\right)=O\left(n^{1 / 6}\right)$, we obtain from (2.84)

$$
\begin{aligned}
B^{-1}\left(y_{n}\right) B\left(x_{n}\right) & =E^{-1}\left(y_{n}\right)\left(I+O\left(\frac{x-y}{n}\right)\right) E\left(x_{n}\right) \\
& =E^{-1}\left(y_{n}\right) E\left(x_{n}\right)+O\left(n^{1 / 6}\right) O\left(\frac{x-y}{n}\right) O\left(n^{1 / 6}\right) \\
& =I+O\left(\frac{x-y}{n^{1 / 3}}\right) .
\end{aligned}
$$

This completes the proof of part (c).

Proof of Theorems 1.1 and 1.2. We let $n, N \rightarrow \infty, t=n / N \rightarrow 1$, in such a way that $n^{2 / 3}(t-1) \rightarrow L$. Then by parts (a) and (b) of Lemma 2.11, we have

$$
\vec{\psi}_{\alpha}\left(n^{2 / 3}\left(f_{t}\left(x_{n}\right)-f_{t}(0)\right) ; n^{2 / 3} f_{t}(0)\right) \rightarrow \vec{\psi}_{\alpha}(x ; s)
$$

and similarly if we replace $x_{n}$ by $y_{n}$. The existence of the limit (1.9) then follows easily from Lemma 2.10 and part (c) of Lemma 2.11, which proves Theorem 1.1 .

We also find that the limiting kernel $K_{\alpha}^{e d g e}(x, y ; s)$ is given by

$$
K_{\alpha}^{e d g e}(x, y ; s)=\frac{1}{2 \pi i(x-y)} \vec{\psi}_{\alpha}(y ; s)^{T}\left(\begin{array}{cc}
0 & 1 \\
-1 & 0
\end{array}\right) \vec{\psi}_{\alpha}(x ; s)
$$

and so (1.13) follows because of (2.85). The model RH problem is solvable for every $s \in \mathbb{R}$ by Proposition 2.4 and so we have also proved Theorem 1.2 . 


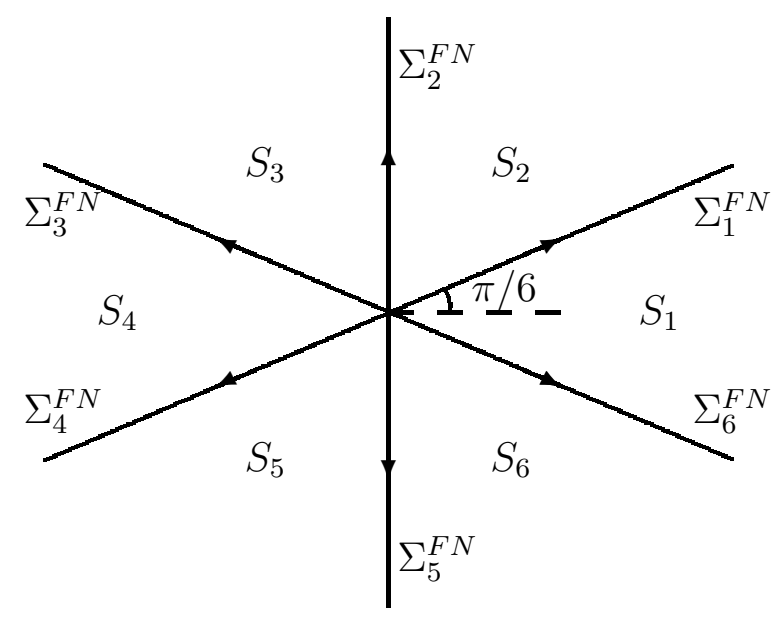

Figure 5: Contour for the RH problem for $\Psi_{\nu}^{F N}$.

\section{Proof of Theorems 1.4 and 1.5}

We prove Theorem 1.4 and Theorem 1.5 by first establishing, with the help of [2], a connection between the model $\mathrm{RH}$ problem and the $\mathrm{RH}$ problem for Painlevé II in the form due to Flaschka and Newell [22]. We can then use known properties of the RH problem for Painlevé II to prove the theorems.

\subsection{The Painlevé II RH problem}

We review the $\mathrm{RH}$ problem for the Painlevé II equation $q^{\prime \prime}(s)=s q+2 q^{3}-\nu$, as first given by Flaschka and Newell [22], see also [24] and [25]. We will assume that

$$
\nu>-1 / 2
$$

The RH problem involves three complex constants $a_{1}, a_{2}, a_{3}$ satisfying

$$
a_{1}+a_{2}+a_{3}+a_{1} a_{2} a_{3}=-2 i \sin \nu \pi
$$

and certain connection matrices $E_{j}$.

Let $S_{j}=\left\{w \in \mathbb{C} \mid \frac{2 j-3}{6} \pi<\arg w<\frac{2 j-1}{6} \pi\right\}$ for $j=1, \ldots, 6$, and let $\Sigma^{F N}=\mathbb{C} \backslash \bigcup_{j} S_{j}$. Then $\Sigma^{F N}$ consists of six rays $\Sigma_{j}^{F N}$ for $j=1, \ldots, 6$, all chosen oriented towards infinity as in Figure 5. The RH problem is the following.

Riemann-Hilbert problem for $\Psi_{\nu}^{F N}$

- $\Psi_{\nu}^{F N}: \mathbb{C} \backslash \Sigma^{F N} \rightarrow \mathbb{C}^{2 \times 2}$ is analytic,

$$
\begin{array}{r}
\bullet \Psi_{\nu,+}^{F N}=\Psi_{\nu,-}^{F N}\left(\begin{array}{cc}
1 & 0 \\
a_{1} & 1
\end{array}\right) \text { on } \Sigma_{1}^{F N}, \\
\Psi_{\nu,+}^{F N}=\Psi_{\nu,-}^{F N}\left(\begin{array}{cc}
1 & a_{2} \\
0 & 1
\end{array}\right) \text { on } \Sigma_{2}^{F N}, \\
\Psi_{\nu,+}^{F N}=\Psi_{\nu,-}^{F N}\left(\begin{array}{cc}
1 & 0 \\
a_{3} & 1
\end{array}\right) \text { on } \Sigma_{3}^{F N},
\end{array}
$$




$$
\begin{aligned}
& \Psi_{\nu,+}^{F N}=\Psi_{\nu,-}^{F N}\left(\begin{array}{cc}
1 & a_{1} \\
0 & 1
\end{array}\right) \text { on } \Sigma_{4}^{F N} \\
& \Psi_{\nu,+}^{F N}=\Psi_{\nu,-}^{F N}\left(\begin{array}{cc}
1 & 0 \\
a_{2} & 1
\end{array}\right) \text { on } \Sigma_{5}^{F N} \\
& \Psi_{\nu,+}^{F N}=\Psi_{\nu,-}^{F N}\left(\begin{array}{cc}
1 & a_{3} \\
0 & 1
\end{array}\right) \text { on } \Sigma_{6}^{F N}
\end{aligned}
$$

- $\Psi_{\nu}^{F N}(w)=(I+O(1 / w)) e^{-i\left(\frac{4}{3} w^{3}+s w\right) \sigma_{3}}$ as $w \rightarrow \infty$.

- If $\nu-\frac{1}{2} \notin \mathbb{N}_{0}$, then

$$
\Psi_{\nu}^{F N}(w)=B(w)\left(\begin{array}{cc}
w^{\nu} & 0 \\
0 & w^{-\nu}
\end{array}\right) E_{j}, \quad \text { for } w \in S_{j}
$$

where $B$ is analytic. If $\nu \in \frac{1}{2}+\mathbb{N}_{0}$, then there exists a constant $\kappa$ such that

$$
\Psi_{\nu}^{F N}(w)=B(w)\left(\begin{array}{cc}
w^{\nu} & \kappa w^{\nu} \log w \\
0 & w^{-\nu}
\end{array}\right) E_{j}, \quad \text { for } w \in S_{j}
$$

where $B$ is analytic.

The connection matrix $E_{1}$ is given explicitly in [24, Chapter 5]. It is determined (up to inessential left diagonal or upper triangular factors) by $\nu$ and the Stokes multipliers $a_{1}, a_{2}$, and $a_{3}$, except in the special case

$$
\nu=\frac{1}{2}+n, \quad a_{1}=a_{2}=a_{3}=i(-1)^{n+1}, \quad n \in \mathbb{Z}
$$

where an additional parameter $c \in \mathbb{C} \cup\{\infty\}$ is needed. For example, for $\nu \notin \frac{1}{2}+\mathbb{N}_{0}$ and $1+a_{1} a_{2} \neq 0$, we have

$$
E_{1}=\left(\begin{array}{cc}
d & 0 \\
0 & d^{-1}
\end{array}\right)\left(\begin{array}{cc}
1 & \frac{e^{-\nu \pi i}-a_{2}}{1+a_{1} a_{2}} \\
-\frac{1+a_{1} a_{2}}{2 \cos \nu \pi} & \frac{e^{\nu \pi i}+a_{2}}{2 \cos \nu \pi}
\end{array}\right),
$$

where $d \neq 0$ is arbitrary. In the special case (3.4), when $E_{1}$ depends on the additional parameter $c \in \mathbb{C} \cup\{\infty\}$, by [24, Chapter 5, (5.0.21)] we may take $E_{1}$ as

$$
E_{1}=\left(\begin{array}{ll}
1 & 0 \\
c & 1
\end{array}\right), \quad \text { if } c \in \mathbb{C}, \quad \text { while } \quad E_{1}=\left(\begin{array}{cc}
0 & -1 \\
1 & 0
\end{array}\right) \quad \text { if } c=\infty
$$

Assuming that the branch cuts for the functions in (3.2) and (3.3) are chosen along $\arg w=-\pi / 6$, we obtain the other connection matrices from $E_{1}$ through the formula

$$
E_{j+1}=E_{j} v_{j}^{F N}, \quad j=1, \ldots, 5,
$$

where $v_{j}^{F N}$ is the jump matrix on $\Sigma_{j}^{F N}$. We shall refer to the Stokes multipliers $a_{1}, a_{2}$, and $a_{3}$, and in the special case (3.4) also to the additional parameter $c$, as the monodromy data for Painlevé II. We note that in the special case (3.4) we have $\kappa=0$ in (3.3). 
The special case (3.4) has geometric interpretation. Indeed, (3.4) describes the singular point of the algebraic variety (3.1), that is, the point at which the (complex) gradient of the left-hand side of (3.1) vanishes. The singularity may be removed by attaching a copy of the Riemann sphere (see also [30]).

The monodromy data does not depend on $s$. The RH problem is uniquely solvable, except for a discrete set of $s$-values, and its solution $\Psi_{\nu}^{F N}$ depends on $s$ through the asymptotic condition at infinity. We write $\Psi_{\nu}^{F N}(w ; s)$ if we want to emphasize its dependence on $s$. If we take

$$
q(s)=2 i \lim _{w \rightarrow \infty} w\left(\Psi_{\nu}^{F N}(w ; s)\right)_{12} e^{i\left(\frac{4}{3} w^{3}+s w\right) \sigma_{3}},
$$

then $q$ satisfies the Painlevé II equation $q^{\prime \prime}=s q+2 q^{3}-\nu$. In addition $\Psi_{\nu}^{F N}$ satisfies the Lax pair for Painlevé II

$$
\begin{aligned}
& \frac{\partial}{\partial w} \Psi=L \Psi, \quad L=\left(\begin{array}{cc}
-4 i w^{2}-i\left(s+2 q^{2}\right) & 4 w q+2 i r+\frac{\nu}{w} \\
4 w q-2 i r+\frac{\nu}{w} & 4 i w^{2}+i\left(s+2 q^{2}\right)
\end{array}\right), \\
& \frac{\partial}{\partial s} \Psi=P \Psi, \quad P=\left(\begin{array}{cc}
-i w & q \\
q & i w
\end{array}\right),
\end{aligned}
$$

where $q=q(s)$ and $r=r(s)=q^{\prime}(s)$. In this way there is a one-to-one correspondence between monodromy data and solutions of Painlevé II.

We also need the more precise asymptotic behavior

$$
\Psi_{\nu}^{F N}(w ; s)=\left(I+\frac{1}{2 i w}\left(\begin{array}{cc}
H(s) & q(s) \\
-q(s) & -H(s)
\end{array}\right)+O\left(1 / w^{2}\right)\right) e^{-i\left(\frac{4}{3} w^{3}+s w\right) \sigma_{3}}
$$

as $w \rightarrow \infty$, where

$$
H(s)=\left(q^{\prime}(s)\right)^{2}-s q^{2}(s)-q^{4}(s)+2 \nu q(s)
$$

is the Hamiltonian for Painlevé II. Note that $H^{\prime}=-q^{2}$.

We finally note that $\Psi_{\nu}^{F N}$ satisfies the symmetry property

$$
\Psi_{\nu}^{F N}(w ; s)=\sigma_{1} \Psi_{\nu}^{F N}(-w ; s) \sigma_{1}
$$

where $\sigma_{1}=\left(\begin{array}{ll}0 & 1 \\ 1 & 0\end{array}\right)$. Indeed, by a straightforward calculation (see also [24, Chapter 5]) we check that the function $\sigma_{1} \Psi_{\nu}^{F N}(-w ; s) \sigma_{1}$ solves exactly the same RH problem as the function $\Psi_{\nu}^{F N}(w ; s)$. Unique solvability of the $\mathrm{RH}$ problem yields equation (3.13).

\subsection{Connection with $\Psi_{\alpha}$}

The Hastings-McLeod solution of Painlevé II corresponds to the Stokes multipliers $a_{1}=$ $-e^{\nu \pi i}, a_{2}=0$, and $a_{3}=e^{-\nu \pi i}$. This is not the solution that interests us here. We use instead the solution corresponding to

$$
a_{1}=e^{-\nu \pi i}, \quad a_{2}=-i, \quad a_{3}=-e^{\nu \pi i} .
$$

For these Stokes multiplies (3.14) we obtain from (3.5) the following connection matrix $E_{1}$ in case $\nu \notin \frac{1}{2}+\mathbb{N}_{0}$ (where we take $d=\left(e^{\nu \pi i}-i\right) /(2 \cos \nu \pi)$ )

$$
E_{1}=\left(\begin{array}{cc}
\frac{e^{\nu \pi i}-i}{2 \cos \nu \pi} & \frac{i e^{\nu \pi i}+1}{2 \cos \nu \pi} \\
-e^{-\nu \pi i} & 1
\end{array}\right)
$$


For $\nu \in \frac{3}{2}+2 \mathbb{N}_{0}$, it follows from (3.14) and the formulas in [24, Chapter 5, (5.0.18)] that we can take

$$
E_{1}=\left(\begin{array}{cc}
1 & 0 \\
-i & 1
\end{array}\right)
$$

If $\nu \in \frac{1}{2}+2 \mathbb{N}_{0}$, then we are in the special case (3.4). We then choose $c=i$, so that for $\nu \in \frac{1}{2}+2 \mathbb{N}_{0}$ we have monodromy data

$$
a_{1}=e^{-\nu \pi i}=-i, \quad a_{2}=-i, \quad a_{3}=-e^{\nu \pi i}=-i, \quad c=i
$$

Lemma 3.1 For any $\nu>-1 / 2$, we have that

$$
\left(E_{2}\right)_{21}=\left(E_{3}\right)_{21}=0
$$

Proof. In all cases we may check from (3.14), (3.15), (3.16), (3.17), and (3.6) that the second row of $E_{1}$ is given by $\left(\begin{array}{ll}-a_{1} & 1\end{array}\right)$. So by (3.7) we have that $E_{2}=E_{1}\left(\begin{array}{ll}1 & 0 \\ a_{1} & 1\end{array}\right)$ is upper triangular. Then also $E_{3}=E_{2}\left(\begin{array}{cc}1 & a_{2} \\ 0 & 1\end{array}\right)$ is upper triangular and therefore (3.18) holds.

The following proposition holds for more general monodromy data, and it was established in [2], see also [35]. For the reader's convenience we present a detailed proof for our particular case.

Proposition 3.2 ([2]) For $\alpha>-1 / 2$, let $\Psi_{2 \alpha+1 / 2}^{F N}$ be the unique solution of the RH problem for Painlevé II with parameter $\nu=2 \alpha+1 / 2$ and monodromy data (3.14) in case $\alpha \notin \mathbb{N}_{0}$ (so that $\nu \notin \frac{1}{2}+2 \mathbb{N}_{0}$ ), and monodromy data (3.17) in the special case $\alpha \in \mathbb{N}_{0}$. Then, for any $\eta=\eta(s)$, we have that

$$
\Psi_{\alpha}(\zeta ; s)=\left(\begin{array}{cc}
1 & 0 \\
\eta(s) & 1
\end{array}\right) \zeta^{-\sigma_{3} / 4} \frac{1}{\sqrt{2}}\left(\begin{array}{cc}
1 & i \\
i & 1
\end{array}\right) e^{\pi i \sigma_{3} / 4} \Psi_{2 \alpha+1 / 2}^{F N}\left(w ;-2^{1 / 3} s\right) e^{-\pi i \sigma_{3} / 4}
$$

where $w=e^{\pi i / 2} 2^{-1 / 3} \zeta^{1 / 2}$ with $\operatorname{Im} w>0$, is a solution of the model RH problem for $\Psi_{\alpha}$ given in Subsection 1.2.

Proof. Because of Proposition (2.1) we may take $\eta(s)=0$ without loss of generality.

Clearly $\Psi_{\alpha}$ is analytic on $\mathbb{C} \backslash \Sigma$. The correct asymptotics as $\zeta \rightarrow \infty$ follows immediately, as well as the correct jumps across $\Sigma_{1}, \Sigma_{2}$, and $\Sigma_{4}$. A little bit more work is needed to check the jump across $\Sigma_{3}=(-\infty, 0)$ and the behavior at $z=0$.

In order to analyze the jump across $\Sigma_{3}$, we suppose that $\zeta \in \Sigma_{3}$. Then we have that

$$
w_{+} \equiv e^{\pi i / 2} 2^{-1 / 3} \zeta_{+}^{1 / 2}=-w_{-} \equiv-e^{\pi i / 2} 2^{-1 / 3} \zeta_{-}^{1 / 2} \quad(<0)
$$


and hence by (3.19) and the symmetry property (3.13),

$$
\begin{aligned}
\Psi_{\alpha,+}(\zeta ; s) & =\zeta_{+}^{-\sigma_{3} / 4} \frac{1}{\sqrt{2}}\left(\begin{array}{cc}
1 & i \\
i & 1
\end{array}\right) e^{\pi i \sigma_{3} / 4} \Psi_{2 \alpha+1 / 2}^{F N}\left(w_{+} ;-2^{1 / 3} s\right) e^{-\pi i \sigma_{3} / 4} \\
& =\zeta_{-}^{-\sigma_{3} / 4}\left(\begin{array}{cc}
-i & 0 \\
0 & i
\end{array}\right) \frac{1}{\sqrt{2}}\left(\begin{array}{cc}
1 & i \\
i & 1
\end{array}\right) e^{\pi i \sigma_{3} / 4} \Psi_{2 \alpha+1 / 2}^{F N}\left(-w_{-} ;-2^{1 / 3} s\right) e^{-\pi i \sigma_{3} / 4} \\
& =\zeta_{-}^{-\sigma_{3} / 4} \frac{1}{\sqrt{2}}\left(\begin{array}{cc}
-i & 1 \\
-1 & i
\end{array}\right) e^{\pi i \sigma_{3} / 4} \sigma_{1} \Psi_{2 \alpha+1 / 2}^{F N}\left(w_{-} ;-2^{1 / 3} s\right) \sigma_{1} e^{-\pi i \sigma_{3} / 4} \\
& =\zeta_{-}^{-\sigma_{3} / 4} \frac{1}{\sqrt{2}}\left(\begin{array}{cc}
-i & 1 \\
1 & -i
\end{array}\right) e^{\pi i \sigma_{3} / 4} \Psi_{2 \alpha+1 / 2}^{F N}\left(w_{-} ;-2^{1 / 3} s\right) e^{-\pi i \sigma_{3} / 4}\left(\begin{array}{cc}
0 & i \\
-i & 0
\end{array}\right) \\
& =\zeta_{-}^{-\sigma_{3} / 4} \frac{1}{\sqrt{2}}\left(\begin{array}{cc}
1 & i \\
i & 1
\end{array}\right) e^{\pi i \sigma_{3} / 4} \Psi_{2 \alpha+1 / 2}^{F N}\left(w_{-} ;-2^{1 / 3} s\right) e^{-\pi i \sigma_{3} / 4}\left(\begin{array}{cc}
0 & 1 \\
-1 & 0
\end{array}\right) \\
& =\Psi_{\alpha,-}(\zeta ; s)\left(\begin{array}{cc}
0 & 1 \\
-1 & 0
\end{array}\right) .
\end{aligned}
$$

This shows that $\Psi_{\alpha}$ has the correct jump across $\Sigma_{3}$, and it follows that $\Psi_{\alpha}$ satisfies the parts (a), (b), and (c) of the model RH problem.

Consider now a neighborhood of the point $\zeta=0$. We recall that (3.2) or (3.3) holds with $B(w)$ analytic at 0 . A corollary of the symmetry property (3.13) is the equation

$$
B(w)= \begin{cases}\sigma_{1} B(-w) \sigma_{3}, & \text { if } \nu \notin \frac{1}{2}+\mathbb{N}_{0}, \\
\sigma_{1} B(-w)\left(\begin{array}{cc}
1 & O\left(w^{2 \nu}\right) \\
0 & -1
\end{array}\right) \text { as } w \rightarrow 0, & \text { if } \nu \in \frac{1}{2}+\mathbb{N}_{0},\end{cases}
$$

which yields the formula (cf. [24, Chapter 5])

$$
B(0) \sigma_{3}=\sigma_{1} B(0)
$$

The last relation, together with $\operatorname{det} B(0)=1$, in turn implies that $B(0)$ can be represented in the form

$$
B(0)=\frac{1}{\sqrt{2}}\left(\begin{array}{cc}
1 & -1 \\
1 & 1
\end{array}\right)\left(\begin{array}{cc}
b & 0 \\
0 & b^{-1}
\end{array}\right), \quad b \neq 0 .
$$

If $\zeta \in \Omega_{j}$ then $w \in S_{\pi(j)}, j=1,2,3,4$, where $\pi$ denotes the permutation

$$
\pi=\left(\begin{array}{llll}
1 & 2 & 3 & 4 \\
3 & 4 & 1 & 2
\end{array}\right)
$$

Therefore, for the function $\Psi_{\alpha}(\zeta ; s)$ defined by equation (3.19) with $\eta(s)=0$, we find that (assuming that $\alpha \notin \frac{1}{2} \mathbb{N}_{0}$ )

$$
\begin{aligned}
\Psi_{\alpha}(\zeta ; s) & =\zeta^{-\sigma_{3} / 4} \frac{1}{\sqrt{2}}\left(\begin{array}{cc}
1 & i \\
i & 1
\end{array}\right) e^{\pi i \sigma_{3} / 4} B(0) e^{-\pi i \sigma_{3} / 4}\left(I+O\left(\zeta^{1 / 2}\right)\right) \zeta^{\sigma_{3} / 4} \zeta^{\alpha \sigma_{3}} \widetilde{E}_{\pi(j)} \\
& =\zeta^{-\sigma_{3} / 4} \frac{1}{2}\left(\begin{array}{cc}
1 & i \\
i & 1
\end{array}\right)\left(\begin{array}{cc}
1 & -i \\
-i & 1
\end{array}\right)\left(\begin{array}{cc}
b & 0 \\
0 & b^{-1}
\end{array}\right)\left(I+O\left(\zeta^{1 / 2}\right)\right) \zeta^{\sigma_{3} / 4} \zeta^{\alpha \sigma_{3}} \widetilde{E}_{\pi(j)} \\
& =\zeta^{-\sigma_{3} / 4}\left(I+O\left(\zeta^{1 / 2}\right)\right) \zeta^{\sigma_{3} / 4} \zeta^{\alpha \sigma_{3}}\left(\begin{array}{cc}
b & 0 \\
0 & b^{-1}
\end{array}\right) \widetilde{E}_{\pi(j)} \\
& =O(1) \zeta^{\alpha \sigma_{3}}\left(\begin{array}{cc}
b & 0 \\
0 & b^{-1}
\end{array}\right) \widetilde{E}_{\pi(j)}, \quad \text { as } \zeta \rightarrow 0 \text { in } \Omega_{j},
\end{aligned}
$$


where we have introduced the notation

$$
\widetilde{E}_{j} \equiv e^{\pi i \sigma_{3} / 4}\left(e^{\pi i / 2} 2^{-1 / 3}\right)^{(2 \alpha+1 / 2) \sigma_{3}} E_{j} e^{-\pi i \sigma_{3} / 4} .
$$

From (3.20) it immediately follows that $\Psi_{\alpha}(\zeta ; s)=O\left(\zeta^{-|\alpha|}\right)$ as $\zeta \rightarrow 0$, which is the required behavior in the model $\mathrm{RH}$ problem if $\alpha<0$, or if $\alpha \geq 0$ and $j \in\{2,3\}$. If $\alpha \geq 0$ and $j \in\{1,4\}$, then $\pi(j) \in\{2,3\}$, and it follows from Lemma 3.1 and (3.21) that

$$
\left(\widetilde{E}_{\pi(j)}\right)_{21}=\left(E_{\pi(j)}\right)_{21}=0
$$

Then (3.20) also yields the required behavior of $\Psi_{\alpha}(\zeta ; s)$ as $\zeta \rightarrow 0$ in $\Omega_{1} \cup \Omega_{4}$.

The calculation leading to (3.20) is valid for $\nu \notin \frac{1}{2}+\mathbb{N}_{0}$, or $\alpha \notin \frac{1}{2} \mathbb{N}_{0}$. In fact it is also valid if $\alpha \in \mathbb{N}_{0}$, since then we are in the special case (3.4) where $\kappa=0$ in (3.3) and so no logarithmic terms appear. Logarithmic terms only appear if $\alpha \in \frac{1}{2}+\mathbb{N}_{0}$, and then a similar calculation leads to

$$
\Psi_{\alpha}(\zeta ; s)=O(1) \zeta^{\alpha \sigma_{3}}\left(\begin{array}{cc}
b & 0 \\
0 & b^{-1}
\end{array}\right)\left(\begin{array}{cc}
1 & O(\log \zeta) \\
0 & 1
\end{array}\right) \widetilde{E}_{\pi(j)}
$$

with $\widetilde{E}_{j}$ again given by (3.21). Since $\alpha>0$, the required behavior as $\zeta \rightarrow 0$ then follows in a similar way.

This completes the proof of the proposition.

\subsection{Differential equation}

Recall that $\Psi_{\nu}^{F N}$ has the Lax pair (3.9) and (3.10). Then $\Psi_{\alpha}$ defined by (3.19) also satisfies a system of differential equations. It will involve the solution $q$ of the Painlevé II equation with parameter $\nu=2 \alpha+1 / 2$ and monodromy data (3.14) or (3.17). We put $r=q^{\prime}$ and

$$
\begin{aligned}
& U(s)=q^{2}(s)+r(s)+\frac{s}{2}, \\
& V(s)=q^{2}(s)-r(s)+\frac{s}{2} .
\end{aligned}
$$

The functions $U$ and $V$ both satisfy the Painlevé XXXIV equation in a form similar to (1.19), namely (cf. [24, Chapter 5]):

$$
\begin{aligned}
& U^{\prime \prime}(s)=\frac{\left(U^{\prime}(s)\right)^{2}}{2 U(s)}+2 U^{2}(s)-s U(s)-\frac{(2 \alpha)^{2}}{2 U(s)} \\
& V^{\prime \prime}(s)=\frac{\left(V^{\prime}(s)\right)^{2}}{2 V(s)}+2 V^{2}(s)-s V(s)-\frac{(2 \alpha+1)^{2}}{2 V(s)} .
\end{aligned}
$$

Then we obtain the following differential equations for $\Psi_{\alpha}$.

Lemma 3.3 Let $\Psi_{\alpha}$ be given by (3.19).

(a) If $\eta \equiv 0$, then $\Psi_{\alpha}$ satisfies

$$
\begin{aligned}
& \frac{\partial}{\partial \zeta} \Psi_{\alpha}(\zeta ; s)=A \Psi_{\alpha}(\zeta ; s), \\
& \frac{\partial}{\partial s} \Psi_{\alpha}(\zeta ; s)=B \Psi_{\alpha}(\zeta ; s),
\end{aligned}
$$


where

$$
\begin{aligned}
& A=\left(\begin{array}{cc}
-2^{1 / 3} q\left(-2^{1 / 3} s\right)+\frac{\alpha}{\zeta} & i-i 2^{-1 / 3} U\left(-2^{1 / 3} s\right) \frac{1}{\zeta} \\
-i \zeta+i 2^{-1 / 3} V\left(-2^{1 / 3} s\right) & 2^{1 / 3} q\left(-2^{1 / 3} s\right)-\frac{\alpha}{\zeta}
\end{array}\right), \\
& B=\left(\begin{array}{cc}
-2^{1 / 3} q\left(-2^{1 / 3} s\right) & i \\
-i \zeta & 2^{1 / 3} q\left(-2^{1 / 3} s\right)
\end{array}\right) .
\end{aligned}
$$

(b) For general $\eta$ we have that $\Psi_{\alpha}$ satisfies

$$
\frac{\partial}{\partial \zeta} \Psi_{\alpha}(\zeta ; s)=\left(\begin{array}{cc}
1 & 0 \\
\eta(s) & 1
\end{array}\right) A\left(\begin{array}{cc}
1 & 0 \\
-\eta(s) & 1
\end{array}\right) \Psi_{\alpha}(\zeta ; s),
$$

with A given by (3.28).

Proof. This follows by straightforward calculations from (3.9), (3.10), and (3.19).

The Lax pair (3.26)-(3.27), after the replacement $\zeta \mapsto \zeta-s$, becomes the Lax pair from [2, 35]. Equations (3.24) $-(3.25)$ can also be derived directly from the compatibility conditions of the Lax pair (3.26)-(3.27) in a usual way.

It is a fact [33], that the solution $q$ of the Painlevé II equation (with parameter $\nu=2 \alpha+1 / 2$ and monodromy data (3.14) or (3.17)) has an infinite number of poles on the positive real line, see also (4.29) below. If $-2^{1 / 3} s$ is such a pole then $\Psi_{2 \alpha+1 / 2}^{F N}\left(\cdot,-2^{1 / 3} s\right)$ does not exist. So to be precise, if we assume that $\eta$ is analytic on $\mathbb{R}$, then (3.19) does not define $\Psi_{\alpha}$ for values of $s \in \mathbb{R}$ which belong to the discrete set of values $s$ where $q\left(-2^{1 / 3} s\right)$ has poles.

The relation (3.19) defines $\Psi_{\alpha}$ for all $s \in \mathbb{R}$ only if we are able to choose $\eta$ so that all the poles on the real line of the right-hand side of (3.19) cancel out. Such a choice of $\eta$ would require $\eta$ itself to have poles at the poles of $q\left(-2^{1 / 3} s\right)$.

We will describe two special choices for $\eta$. The first choice is such that (3.19) is equal to the special solution $\Psi_{\alpha}^{(s p e c)}$, which is characterized by the asymptotic condition (2.14). From Proposition 2.4 we know that $\Psi_{\alpha}^{(s p e c)}$ exists for all $s \in \mathbb{R}$, so that we can already conclude that the special choice $\eta=\eta^{(\text {spec })}$ will have poles at the poles of $q\left(-2^{1 / 3} s\right)$, and that the real poles of the right-hand side of (3.19) will indeed cancel out.

The second choice of $\eta$ is made so that the differential equation (3.30) takes a nice form. It will lead to the differential equation (1.21) for $\psi_{1}$ and $\psi_{2}$. This $\eta$ is denoted $\eta_{0}$, and it is defined by the simple formula

$$
\eta_{0}(s)=i 2^{1 / 3} q\left(-2^{1 / 3} s\right),
$$

from which it is already clear that it has poles at the poles of $q\left(-2^{1 / 3} s\right)$. For the choice (3.31) we can already check that the differential equation (3.30) leads to

$$
\frac{\partial}{\partial \zeta} \Psi_{\alpha}(\zeta ; s)=A_{0} \Psi_{\alpha}(\zeta ; s)
$$

where

$$
\begin{aligned}
A_{0} & =\left(\begin{array}{cc}
1 & 0 \\
\eta_{0} & 1
\end{array}\right) A\left(\begin{array}{cc}
1 & 0 \\
-\eta_{0} & 1
\end{array}\right) \\
& =\left(\begin{array}{cc}
\left(\alpha+i u \eta_{0}\right) / \zeta & i-i u / \zeta \\
-i \zeta+i\left(v+\eta_{0}^{2}\right)+\eta_{0}\left(2 \alpha+i u \eta_{0}\right) / \zeta & -\left(\alpha+i u \eta_{0}\right) / \zeta
\end{array}\right),
\end{aligned}
$$


and

$$
\begin{aligned}
& u(s)=2^{-1 / 3} U\left(-2^{1 / 3} s\right), \\
& v(s)=2^{-1 / 3} V\left(-2^{1 / 3} s\right) .
\end{aligned}
$$

\subsection{Special choice $\eta^{(\text {spec })}$}

Lemma 3.4 Let $H$ be the Hamiltonian for Painlevé II as in (3.12), with parameter $\nu=$ $2 \alpha+1 / 2$, and let

$$
\eta^{(\text {spec })}(s)=i 2^{-2 / 3}\left(q\left(-2^{1 / 3} s\right)+H\left(-2^{1 / 3} s\right)\right) .
$$

Then the choice $\eta=\eta^{(\text {spec })}$ in (3.19) leads to the special solution $\Psi_{\alpha}^{(\text {spec })}$ of the model RH problem characterized by (2.14).

Proof. It follows from (3.11) and (3.19) by straightforward computation, that

$$
\begin{aligned}
& \Psi_{\alpha}(\zeta ; s)=\left(\begin{array}{ll}
1 & 0 \\
\eta & 1
\end{array}\right) \zeta^{-\sigma_{3} / 4} \frac{1}{\sqrt{2}}\left(\begin{array}{cc}
1 & i \\
i & 1
\end{array}\right) e^{\pi i \sigma_{3} / 4} \Psi_{2 \alpha+1 / 2}^{F N}\left(w ;-2^{1 / 3} s\right) e^{-\pi i \sigma_{3} / 4} \\
& =\left(\begin{array}{ll}
1 & 0 \\
\eta & 1
\end{array}\right)\left[\left(\begin{array}{cc}
1 & 0 \\
-\eta^{(s p e c)} & 1
\end{array}\right)+\frac{1}{\zeta}\left(\begin{array}{cc}
0 & i 2^{-2 / 3}(H-q)\left(-2^{1 / 3} s\right) \\
0 & 0
\end{array}\right)\right. \\
& \left.+\zeta^{-\sigma_{3} / 4} \frac{1}{\sqrt{2}}\left(\begin{array}{cc}
1 & i \\
i & 1
\end{array}\right) e^{\pi i \sigma_{3} / 4} O(1 / \zeta) e^{-\pi i \sigma_{3} / 4} \frac{1}{\sqrt{2}}\left(\begin{array}{cc}
1 & -i \\
-i & 1
\end{array}\right) \zeta^{\sigma_{3} / 4}\right] \\
& \times \zeta^{-\sigma_{3} / 4} \frac{1}{\sqrt{2}}\left(\begin{array}{ll}
1 & i \\
i & 1
\end{array}\right) e^{-\left(\frac{2}{3} \zeta^{3 / 2}+s \zeta^{1 / 2}\right) \sigma_{3}}
\end{aligned}
$$

as $\zeta \rightarrow \infty$. From (3.37) it is clear that we need to take $\eta=\eta^{(s p e c)}$ in order to be able to obtain (2.14). Thus the lemma follows.

From the calculation (3.37) we also note that for any solution $\Psi_{\alpha}$ of the model RH problem we have

$$
\left(\Psi_{\alpha}(\zeta ; s) e^{\frac{2}{3}\left(\zeta^{3 / 2}+s \zeta^{1 / 2}\right) \sigma_{3}} \frac{1}{\sqrt{2}}\left(\begin{array}{cc}
1 & -i \\
-i & 1
\end{array}\right) \zeta^{\sigma_{3} / 4}\right)_{12}=\frac{i 2^{-2 / 3}(H-q)\left(-2^{1 / 3} s\right)}{\zeta}+O\left(\zeta^{-3 / 2}\right)
$$

as $\zeta \rightarrow \infty$. This property will be used later in the proof of Theorem 1.4 ,

Since the left-hand side of (3.38) is analytic in $s$ for $s \in \mathbb{R}$, it also follows from (3.38) that $H-q$ does not have poles on the real line. This and similar properties are collected in the following lemma. Recall that $U$ is given by (3.22).

Lemma 3.5 The following hold.

(a) $H-q$ has no poles on the real line.

(b) U has no poles on the real line.

(c) $U$ has a zero at each of the real poles of $q$ and $U q$ has no poles on the real line.

(d) Uq takes the value $\nu-1 / 2$ at each of the real poles of $q$. 
Proof. (a) We noted already that part (a) follows from (3.38).

(b) Since $H^{\prime}=-q^{2}$, we have that

$$
U(s)=q^{2}(s)+q^{\prime}(s)+s / 2=-(H-q)^{\prime}(s)+s / 2,
$$

and so it follows from part (a) that $U$ has no poles on the real line either.

(c) Differentiating (3.22), we obtain

$$
U^{\prime}=2 q q^{\prime}+q^{\prime \prime}+\frac{1}{2}=2 q q^{\prime}+s q+2 q^{3}-\nu+\frac{1}{2}=2 U q-\nu+\frac{1}{2} .
$$

Thus also $U q$ has no poles on the real line, which means that $U$ has a zero at each of the real poles of $q$.

(d) Using (3.40), we get

$$
\left(U q-\nu+\frac{1}{2}\right) q=\left(U^{\prime}-U q\right) q=(U q)^{\prime}-U\left(q^{2}+q^{\prime}\right)=(U q)^{\prime}-U(U-s / 2) .
$$

Since the right-hand side of (3.41) is analytic on the real line by parts (b) and (c), we conclude that $U q-\nu+\frac{1}{2}$ has a zero at each of the real poles of $q$. This proves part (d).

It is well-known and easy to check that each pole of $q$ is simple and has residue +1 or -1 . Indeed, the Laurent series for $q$ at a pole $s_{0}$ has the form

$$
q(s)=\frac{q_{-1}}{s-s_{0}}+q_{1}\left(s-s_{0}\right)+\cdots
$$

where $q_{-1} \in\{-1,1\}$. Using this, one easily verifies that either $q^{2}+q^{\prime}$ or $q^{2}-q$ is analytic at $s_{0}$ (depending on the sign of the residue $q_{-1}$ ). Our result that $U=q^{2}+q^{\prime}+s / 2$ is analytic on $\mathbb{R}$ can then also be stated as follows.

Corollary 3.6 The solution $q$ of the Painlevé II equation with parameter $\nu=2 \alpha+1 / 2$ and monodromy data (3.14) or (3.17) has only simple poles on the real line, with residue +1 .

\subsection{Special choice $\eta_{0}$}

As already announced we will also use the special choice $\eta=\eta_{0}$ given by (3.31).

By (3.31) and (3.36) we have that

$$
\eta_{0}(s)-\eta^{(\text {spec })}(s)=i 2^{-2 / 3}\left(q\left(-2^{1 / 3} s\right)-H\left(-2^{1 / 3} s\right)\right),
$$

and so it follows from part (a) of Lemma 3.5 that $\eta_{0}-\eta^{(\text {spec })}$ is analytic on the real line. Since $\Psi_{\alpha}^{(s p e c)}$ exists for all $s \in \mathbb{R}$, it follows that the solution of the model RH problem associated with $\eta_{0}$ exists for all $s \in \mathbb{R}$ as well, and it is analytic in $s$.

The differential equation for $\Psi_{\alpha}$ with $\eta=\eta_{0}$ is given by (3.32) with $A_{0}$ as in (3.33). It then follows that $A_{0}$ is analytic on the real line, and we will explicitly verify this by rewriting its entries in terms of the function $u$ from (3.34)

$$
u(s)=2^{-1 / 3} U\left(-2^{1 / 3} s\right) .
$$


The analyticity of $u$ is immediate from (3.34) and part (b) of Lemma 3.5. The analyticity of $u \eta_{0}$ follows from (3.34), (3.31) and part (c) of Lemma 3.5. Using also (3.40) we get

$$
u^{\prime}=2 i u \eta_{0}+\nu-1 / 2=2 i u \eta_{0}+2 \alpha .
$$

Next, it follows from (3.22), (3.23), (3.34), (3.35)), and (3.31) that

$$
v(s)+\eta_{0}(s)^{2}=-u(s)-s .
$$

We can use (3.42) and (3.43) to eliminate $\eta_{0}$ and $v$ from the entries in $A_{0}$, and we get from (3.33) that

$$
A_{0}=\left(\begin{array}{cc}
u^{\prime} /(2 \zeta) & i-i u / \zeta \\
-i \zeta-i(u+s)-i\left(\left(u^{\prime}\right)^{2}-(2 \alpha)^{2}\right) /(4 u \zeta) & -u^{\prime} /(2 \zeta)
\end{array}\right)
$$

\subsection{Proof of Theorem 1.4 and 1.5}

After these preparations the proofs of Theorems 1.4 and 1.5 are short.

Proof of Theorem 1.4. From (3.24) and (3.34) it follows that $u$ satisfies the Painlevé XXXIV equation in the form (1.19).

From (3.38) it follows that

$$
\lim _{\zeta \rightarrow \infty}\left[\zeta\left(\Psi_{\alpha}(\zeta ; s) e^{\frac{2}{3}\left(\zeta^{3 / 2}+s \zeta^{1 / 2}\right) \sigma_{3}} \frac{1}{\sqrt{2}}\left(\begin{array}{cc}
1 & -i \\
-i & 1
\end{array}\right) \zeta^{\sigma_{3} / 4}\right)_{12}\right]=i 2^{-2 / 3}(H-q)\left(-2^{1 / 3} s\right)
$$

which in view of (3.39) and (3.34) leads to (1.20). This proves Theorem 1.4.

Proof of Theorem 1.5. Let $\Psi_{\alpha}$ be the solution of the model RH problem given by (3.19) with $\eta=\eta_{0}$ as in (3.31). Then

$$
\frac{\partial}{\partial \zeta} \Psi_{\alpha}(\zeta ; s)=A_{0} \Psi_{\alpha}(\zeta ; s)
$$

with $A_{0}$ given by (3.44). The differential equation (3.45) is valid for $\zeta \in \mathbb{C} \backslash \Sigma$. We can take the limit $\zeta \rightarrow x$ with $x \in \mathbb{R} \backslash\{0\}$ to obtain a differential equation for $\Psi_{\alpha,+}(x ; s)$, with the same matrix $A_{0}$ (but with $\zeta$ replaced by $x$ ). Using (1.12), we obtain the differential equation (1.21) for $\psi_{1}$ and $\psi_{2}$. This completes the proof of Theorem 1.5.

\section{Concluding remarks}

\subsection{The case $\alpha=0$}

The case $\alpha=0$ is classical and well understood. We know that $K_{0}^{\text {edge }}(x, y ; s)$ is the (shifted) Airy kernel, see (1.11). We will show here how this follows from the calculations from the previous section.

In the special case $\alpha=0$, we have $\nu=1 / 2$, and then the Painlevé II equation has special solutions built out of Airy functions. To be precise if $\mathrm{Ai}$ and $\mathrm{Bi}$ are the standard Airy functions, then for any $C_{1}$ and $C_{2}$, not both zero, we have that

$$
q(s)=\frac{d}{d s} \log \left(C_{1} \operatorname{Ai}\left(-2^{-1 / 3} s\right)+C_{2} \operatorname{Bi}\left(-2^{-1 / 3} s\right)\right)
$$


is a solution of $q^{\prime \prime}=s q+2 q^{3}-\frac{1}{2}$. These are exactly the solutions that correspond to the special Stokes multipliers $a_{1}=a_{2}=a_{3}=-i$. The corresponding solutions to the $\mathrm{RH}$ problem were given by Flaschka and Newell [22, Section 3F(iv)]. For example, for $w$ in sector $S_{1}$ we have (see also [24, Chapter 11])

$$
\Psi_{1 / 2}^{F N}(w ; s)=\frac{\alpha_{0}}{2} w^{1 / 2}\left(\begin{array}{cc}
1-i q(s) / w & -2^{-1 / 3} i / w \\
1+i q(s) / w & 2^{-1 / 3} i / w
\end{array}\right)\left(\begin{array}{cc}
\operatorname{Ai}(z) & \operatorname{Bi}(z) \\
\operatorname{Ai}^{\prime}(z) & \operatorname{Bi}^{\prime}(z)
\end{array}\right)\left(\begin{array}{cc}
-i & 1 \\
1 & -i
\end{array}\right)
$$

with $z=-2^{2 / 3} w^{2}-2^{-1 / 3} s$ and $\alpha_{0}=2^{1 / 6} \sqrt{\pi} e^{i \pi / 4}$. The expressions for $\Psi_{\nu}^{F N}(w ; s)$ in the other sectors follow by multiplying (4.2) by the appropriate jump matrices.

It follows from (4.1) and (4.2) that the extra parameter $c$ in the monodromy data for (4.1) is

$$
c=\frac{i C_{1}-C_{2}}{C_{1}-i C_{2}}
$$

So if we take $c=i$ as in (3.17) then $C_{2}=0$ and the corresponding solution (4.1) is

$$
q(s)=\frac{d}{d s} \log \operatorname{Ai}\left(-2^{-1 / 3} s\right)=-2^{-1 / 3} \frac{\operatorname{Ai}^{\prime}\left(-2^{-1 / 3} s\right)}{\operatorname{Ai}\left(-2^{-1 / 3} s\right)} .
$$

Note that the solution (4.4) is special among all solutions (4.1) in its behavior for $s \rightarrow-\infty$. Indeed, from the asymptotic behavior for the Airy functions it follows that for (4.4) we have

$$
q(s) \sim \frac{1}{2} \sqrt{2}(-s)^{1 / 2} \quad \text { as } s \rightarrow-\infty
$$

while for the other solutions (4.1) we have

$$
q(s) \sim-\frac{1}{2} \sqrt{2}(-s)^{1 / 2} \quad \text { as } s \rightarrow-\infty .
$$

So according to Proposition 3.2 we should be using $q$ given by (4.4) and then define $\Psi_{0}$ as in (3.19). If $\zeta$ is in sector $\Omega_{3}$, then $w=e^{i \pi / 2} 2^{-1 / 3} \zeta^{1 / 2}$ is in sector $S_{1}$, so that by (4.2)

$$
\Psi_{1 / 2}^{F N}\left(w ;-2^{1 / 3} s\right)=\frac{\sqrt{\pi} i}{2} \zeta^{1 / 4}\left(\begin{array}{cc}
1+i \eta_{0}(s) \zeta^{-1 / 2} & -\zeta^{-1 / 2} \\
1-i \eta_{0}(s) \zeta^{-1 / 2} & \zeta^{-1 / 2}
\end{array}\right)\left(\begin{array}{cc}
\operatorname{Ai}(\zeta+s) & \operatorname{Bi}(\zeta+s) \\
\operatorname{Ai}^{\prime}(\zeta+s) & \operatorname{Bi}^{\prime}(\zeta+s)
\end{array}\right)\left(\begin{array}{cc}
-i & 1 \\
1 & -i
\end{array}\right)
$$

where $\eta_{0}(s)=i 2^{1 / 3} q\left(-2^{1 / 3}\right)$ as in (3.31). Then (3.19) with $\eta=\eta_{0}$ yields for $\zeta \in \Omega_{3}$,

$$
\begin{aligned}
\Psi_{0}(\zeta ; s)= & \frac{\sqrt{\pi}}{2 \sqrt{2}}\left(\begin{array}{cc}
1 & 0 \\
\eta_{0}(s) & 1
\end{array}\right)\left(\begin{array}{cc}
1 & 0 \\
0 & \zeta^{1 / 2}
\end{array}\right)\left(\begin{array}{cc}
1 & i \\
i & 1
\end{array}\right) e^{\pi i \sigma_{3} / 4} \\
& \times\left(\begin{array}{cc}
1+i \eta_{0}(s) \zeta^{-1 / 2} & -\zeta^{-1 / 2} \\
1-i \eta_{0}(s) \zeta^{-1 / 2} & \zeta^{-1 / 2}
\end{array}\right)\left(\begin{array}{cc}
\operatorname{Ai}(\zeta+s) & \operatorname{Bi}(\zeta+s) \\
\operatorname{Ai}^{\prime}(\zeta+s) & \operatorname{Bi}^{\prime}(\zeta+s)
\end{array}\right)\left(\begin{array}{cc}
1 & i \\
i & 1
\end{array}\right) e^{-\pi i \sigma_{3} / 4} \\
= & \frac{\sqrt{\pi}}{\sqrt{2}} e^{\pi i \sigma_{3} / 4}\left(\begin{array}{cc}
\operatorname{Ai}(\zeta+s) & \operatorname{Bi}(\zeta+s) \\
\operatorname{Ai}^{\prime}(\zeta+s) & \operatorname{Bi}^{\prime}(\zeta+s)
\end{array}\right)\left(\begin{array}{cc}
1 & i \\
i & 1
\end{array}\right) e^{-\pi i \sigma_{3} / 4} \\
= & \frac{\sqrt{\pi}}{\sqrt{2}}\left(\begin{array}{cc}
\operatorname{Ai}(\zeta+s)+i \operatorname{Bi}(\zeta+s) & -\left(\operatorname{Ai}^{\prime}(\zeta+s)-i \operatorname{Bi}(\zeta+s)\right) \\
-i\left(\operatorname{Ai}^{\prime}(\zeta+s)+i \operatorname{Bi}^{\prime}(\zeta+s)\right) & i\left(\operatorname{Ai}^{\prime}(\zeta+s)-i \operatorname{Bi}^{\prime}(\zeta+s)\right)
\end{array}\right)
\end{aligned}
$$


Since (see e.g. formula (10.4.9) in [1])

$$
\operatorname{Ai}(z) \pm i \operatorname{Bi}(z)=2 e^{ \pm \pi i / 3} \operatorname{Ai}\left(e^{\mp 2 \pi i / 3} z\right)
$$

we can write $\Psi_{0}$ in the more familiar form

$$
\Psi_{0}(\zeta ; s)=\sqrt{2 \pi}\left(\begin{array}{cc}
e^{\pi i / 3} \operatorname{Ai}\left(e^{-2 \pi i / 3}(\zeta+s)\right) & -e^{-\pi i / 3} \operatorname{Ai}\left(e^{2 \pi i / 3}(\zeta+s)\right) \\
-i e^{-\pi i / 3} \operatorname{Ai}^{\prime}\left(e^{-2 \pi i / 3}(\zeta+s)\right) & i e^{\pi i / 3} \operatorname{Ai}^{\prime}\left(e^{2 \pi i / 3}(\zeta+s)\right)
\end{array}\right), \quad \text { for } \zeta \in \Omega_{3} .
$$

For $\zeta \in \Omega_{1}$ we find in a similar way (or by multiplying (4.5) on the right by $\left(\begin{array}{cc}1 & 1 \\ -1 & 0\end{array}\right)$ ), that

$$
\Psi_{0}(\zeta ; s)=\sqrt{2 \pi}\left(\begin{array}{cc}
\operatorname{Ai}(\zeta+s) & e^{\pi i / 3} \operatorname{Ai}\left(e^{-2 \pi i / 3}(\zeta+s)\right) \\
-i \operatorname{Ai}^{\prime}(\zeta+s) & -i e^{-\pi i / 3} \operatorname{Ai}^{\prime}\left(e^{-2 \pi i / 3}(\zeta+s)\right)
\end{array}\right), \quad \text { for } \zeta \in \Omega_{1} .
$$

Then it follows from (1.12) and (4.6) that for $x>0$,

$$
\psi_{1}(x ; s)=\sqrt{2 \pi} \operatorname{Ai}(x+s), \quad \psi_{2}(x ; s)=-\sqrt{2 \pi} i \mathrm{Ai}^{\prime}(x+s),
$$

and a similar calculation shows that (4.7) also holds for $x<0$. Therefore, by (1.13)

$$
\begin{aligned}
K_{0}^{e d g e}(x, y ; s) & =\frac{\psi_{2}(x ; s) \psi_{1}(y ; s)-\psi_{1}(x ; s) \psi_{2}(y ; s)}{2 \pi i(x-y)} \\
& =\frac{\operatorname{Ai}(x+s) \operatorname{Ai}^{\prime}(y+s)-\operatorname{Ai}^{\prime}(x+s) \operatorname{Ai}(y+s)}{x-y}
\end{aligned}
$$

which is indeed the (shifted) Airy kernel.

\subsection{The case $\alpha=1$}

The case $\alpha=1$ can be solved explicitly in terms of Airy functions as well. Let $\Psi_{0}$ be a solution of the model RH problem with parameter $\alpha=0$. Then for any matrix $X=X(s)$, it is easy to check that

$$
\Psi_{1}(\zeta ; s)=\left(I-\frac{1}{\zeta} X(s)\right) \Psi_{0}(\zeta ; s)
$$

satisfies the conditions (a), (b), and (c) of the model RH problem for $\alpha=1$. For a special choice of $X$ we will have that the condition $(d)$ is also satisfied.

Let's take $\Psi_{0}$ given by (4.6) for $\zeta \in \Omega_{1}$. Then the condition (d) of the model RH problem yields the following condition on $X$

$$
\left(I-\frac{1}{\zeta} X(s)\right)\left(\begin{array}{c}
\mathrm{Ai}(\zeta+s) \\
-i \mathrm{Ai}^{\prime}(\zeta+s)
\end{array}\right)=O(\zeta), \quad \text { as } \zeta \rightarrow 0 .
$$

The condition (4.10) is satisfied if and only if we take

$$
X(s)=\frac{1}{\operatorname{Ai}^{\prime}(s)^{2}-s \operatorname{Ai}(s)^{2}}\left(\begin{array}{c}
\operatorname{Ai}(s) \\
-i \mathrm{Ai}^{\prime}(s)
\end{array}\right)\left(\operatorname{Ai}^{\prime}(s)-i \operatorname{Ai}(s)\right) .
$$

Note that the denominator in (4.11) cannot be zero for $s \in \mathbb{R}$. Indeed, its derivative is $-\operatorname{Ai}(s)^{2}$, so that it is decreasing for $s \in \mathbb{R}$, and since the limit for $s \rightarrow+\infty$ is equal to 
0 , it follows that $\mathrm{Ai}^{\prime}(s)-s \mathrm{Ai}(s)^{2}>0$ for all $s \in \mathbb{R}$. Note also that if we take the limit $x, y \rightarrow 0$ in (4.8), then

$$
K_{0}^{\text {edge }}(0,0 ; s)=\operatorname{Ai}^{\prime}(s)^{2}-s \operatorname{Ai}(s)^{2} .
$$

Using (1.12), (4.6), (4.9), (4.11), and (4.12), we obtain that

$$
\begin{aligned}
\psi_{1}(x ; s) & =\sqrt{2 \pi} \operatorname{Ai}(x+s)-\sqrt{2 \pi} \frac{\operatorname{Ai}(x+s) \operatorname{Ai}^{\prime}(s)-\operatorname{Ai}(s) \operatorname{Ai}^{\prime}(x+s)}{x\left(\operatorname{Ai}^{\prime}(s)^{2}-s \operatorname{Ai}(s)^{2}\right)} \operatorname{Ai}(s) \\
& =\sqrt{2 \pi}\left(\operatorname{Ai}(x+s)-\frac{K_{0}^{e d g e}(x, 0 ; s)}{K_{0}^{e d g e}(0,0 ; s)} \operatorname{Ai}(s)\right) \\
\psi_{2}(x ; s) & =-\sqrt{2 \pi} i \operatorname{Ai}^{\prime}(x+s)+\sqrt{2 \pi} i \frac{\operatorname{Ai}(x+s) \operatorname{Ai}^{\prime}(s)-\operatorname{Ai}(s) \operatorname{Ai}^{\prime}(x+s)}{x\left(\operatorname{Ai}^{\prime}(s)^{2}-s \operatorname{Ai}(s)^{2}\right)} \operatorname{Ai}^{\prime}(s) \\
& =-\sqrt{2 \pi} i\left(\operatorname{Ai}^{\prime}(x+s)-\frac{K_{0}^{e d g e}(x, 0 ; s)}{K_{0}^{e d g e}(0,0 ; s)} \operatorname{Ai}^{\prime}(s)\right) .
\end{aligned}
$$

Thus

$$
\begin{aligned}
K_{1}^{\text {edge }}(x, y ; s) & =\frac{\psi_{2}(x ; s) \psi_{1}(y ; s)-\psi_{1}(x ; s) \psi_{2}(y ; s)}{2 \pi i(x-y)} \\
& =K_{0}^{e d g e}(x, y ; s)-\frac{K_{0}^{e d g e}(x, 0 ; s) K_{0}^{e d g e}(y, 0 ; s)}{K_{0}^{\text {edge }}(0,0 ; s)} .
\end{aligned}
$$

To compute the relevant solution $u$ of the Painlevé XXXIV equation for $\alpha=1$, we may assume that we have taken $\Psi_{0}^{\text {spec }}$ in (4.9), and then use (1.20), (4.9), (2.14), and the fact that $u \equiv 0$ for $\alpha=0$, to obtain that $u(s)=i X_{12}^{\prime}(s)$, which by (4.11) leads to

$$
u(s)=\frac{d}{d s}\left(\frac{\operatorname{Ai}(s)^{2}}{\operatorname{Ai}^{\prime}(s)^{2}-s \operatorname{Ai}(s)^{2}}\right)=-\frac{d^{2}}{d s^{2}} \log K_{0}^{\text {edge }}(0,0 ; s) .
$$

Its graph is shown in Figure 6.

One can verify from the explicitly known asymptotic formulas for Ai that

$$
u(s)=\frac{1}{\sqrt{s}}-\frac{1}{s^{2}}+O\left(s^{-7 / 2}\right) \quad \text { as } s \rightarrow+\infty .
$$

On the negative real axis, $u$ has an infinite number of zeros. These are the zeros of the Airy function $\mathrm{Ai}$, and an infinite number of additional zeros that interlace with the zeros of Ai.

Equations (4.9) and (4.14) constitute the Schlesinger and (induced by it) Bäcklund transformations, respectively, for the case of Painlevé XXXIV and applied to its zero vacuum solution (for the general theory of Schlesinger transformations see [31]; see also [24, Chapter 6]).

\subsection{Asymptotic characterization of the Painlevé function $u(s)$}

We finally want to characterize the solution $u$ of the Painlevé XXXIV equation by its asymptotic properties. Recall that $u$ is connected to the solution of the Painlevé II equation $q^{\prime \prime}=s q+2 q^{3}-\nu$ with $\nu=2 \alpha+1 / 2$ by the formulas

$$
u(s)=2^{-1 / 3} U\left(-2^{1 / 3} s\right), \quad U(s)=q^{2}(s)+q^{\prime}(s)+\frac{s}{2} .
$$




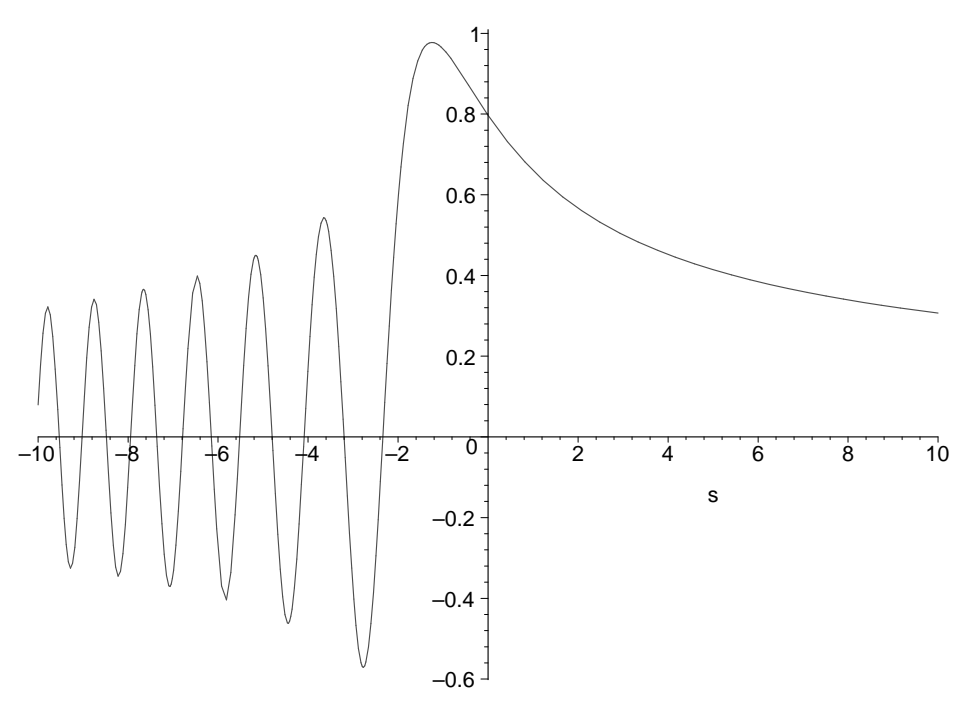

Figure 6: The solution of the Painlevé XXXIV equation for $\alpha=1$.

Assume that $\nu>-1 / 2$. It is shown in [34] (see also [24, Chapters 5, 11]) that the solution $q(s)$ of the Painlevé II equation corresponding to the Stokes multipliers (3.14) exhibits the following asymptotic behavior in the sector $\arg s \in\left[\frac{2 \pi}{3}, \frac{4 \pi}{3}\right]$

$$
\begin{aligned}
q(s)=\sqrt{-\frac{s}{2}} \sum_{n=0}^{[\nu+1 / 2]} b_{n}(-s)^{-3 n / 2}+O\left(s^{-3[\nu+1 / 2] / 2-1}\right) \\
+c_{+}(-s)^{-\frac{3}{2} \nu-\frac{1}{4}} e^{-\frac{2 \sqrt{2}}{3}(-s)^{3 / 2}}\left(1+O\left(s^{-1 / 4}\right)\right), \\
\quad \operatorname{as~} s \rightarrow \infty, \quad \arg s \in\left(\frac{2 \pi}{3}, \frac{4 \pi}{3}\right], \quad \arg (-s) \in\left(-\frac{\pi}{3}, \frac{\pi}{3}\right],
\end{aligned}
$$

or

$$
\begin{aligned}
& q(s)=\sqrt{-\frac{s}{2}} \sum_{n=0}^{[\nu+1 / 2]} b_{n}(-s)^{-3 n / 2}+O\left(s^{-3[\nu+1 / 2] / 2}\right) \\
& +c_{-}(-s)^{-\frac{3}{2} \nu-\frac{1}{4}} e^{-\frac{2 \sqrt{2}}{3}(-s)^{3 / 2}}\left(1+O\left(s^{-1 / 4}\right)\right), \\
& \quad \text { as } s \rightarrow \infty, \quad \arg s \in\left[\frac{2 \pi}{3}, \frac{4 \pi}{3}\right), \quad \arg (-s) \in\left[-\frac{\pi}{3}, \frac{\pi}{3}\right),
\end{aligned}
$$

where we have used the notation $[r]$ for the integer part of the positive number $r$, i.e.

$$
[r] \in \mathbb{N}_{0}, \quad[r] \leq r<[r]+1 .
$$

The coefficients $c_{+}$and $c_{-}$of the exponential terms, which oscillate on the respective boundaries of the sector $\left(\frac{2 \pi}{3}, \frac{4 \pi}{3}\right)$, are given by the formulae

$$
c_{+}=-\frac{e^{\pi\left(\nu+\frac{1}{2}\right) i}+1}{\pi} 2^{-\frac{5}{2} \nu-\frac{7}{4}} \Gamma\left(\frac{1}{2}+\nu\right),
$$

and

$$
c_{-}=-\frac{e^{-\pi\left(\nu+\frac{1}{2}\right) i}+1}{\pi} 2^{-\frac{5}{2} \nu-\frac{7}{4}} \Gamma\left(\frac{1}{2}+\nu\right),
$$


where $\Gamma$ denotes the Gamma function. Moreover, either the relations (4.17), (4.19) or the relations (4.18), (4.20) can be taken as a characterization of the solution $q(s)$.

Alternatively, the solution $q(s)$ can be characterized by its comparison to the Boutroux tri-tronquée solution $q^{(\text {tri-tronq }}(s)$ of the Painlevée II equation, which is defined as the unique solution satisfying the asymptotic condition

$$
\begin{aligned}
q^{(\text {tri-tronq })}(s) \sim \sqrt{-\frac{s}{2}} \sum_{n=0}^{\infty} b_{n}(-s)^{-3 n / 2} \\
\quad \text { as } s \rightarrow \infty, \quad \arg s \equiv \pi+\arg (-s) \in\left(0, \frac{4 \pi}{3}\right) .
\end{aligned}
$$

The solution $q(s)$ we are working with is the one whose asymptotic behavior as $s \rightarrow-\infty$ is given by the equation

$$
\begin{aligned}
q(s)-q^{(\text {tri-tronq })}(s)=- & \frac{e^{-\pi\left(\nu+\frac{1}{2}\right) i}+1}{\pi} 2^{-\frac{5}{2} \nu-\frac{7}{4}} \Gamma\left(\frac{1}{2}+\nu\right) \\
& \quad \times|s|^{-\frac{3}{2} \nu-\frac{1}{4}} e^{-\frac{2 \sqrt{2}}{3}|s|^{3 / 2}}\left(1+O\left(s^{-1 / 4}\right)\right), \quad \text { as } s \rightarrow-\infty .
\end{aligned}
$$

The coefficients $b_{n}$ of the asymptotic series in (4.17), (4.18), and (4.21) are determined by substitution into the Painlevé II equation. Indeed, the following recurrence relation takes place

$$
\left\{\begin{array}{l}
b_{0}=1, \quad b_{1}=\frac{\nu}{\sqrt{2}} \\
b_{n+2}=\frac{9 n^{2}-1}{8} b_{n}-\sum_{m=1}^{n+1} b_{m} b_{n+2-m}-\frac{1}{2} \sum_{l=1}^{n+1} \sum_{m=1}^{n+2-l} b_{l} b_{m} b_{n+2-l-m} .
\end{array}\right.
$$

Using relation (4.16) between the Painlevé II and Painlevé XXXIV functions we arrive at the asymptotic characterization of the function $u(s)$ of Theorem 1.5 .

Proposition 4.1 The solution $u(s)$ of the Painleve XXXIV equation which appears in Theorem 1.5 is uniquely characterized by one of the following asymptotic conditions

$$
\begin{array}{r}
u(s)=\frac{\alpha}{\sqrt{s}}+\sum_{n=1}^{[2 \alpha+1]} a_{n} s^{-\frac{3 n+1}{2}}+O\left(s^{-3[2 \alpha+1] / 2-1}\right) \\
+d_{+} s^{-3 \alpha+\frac{1}{2}} e^{-\frac{4}{3} s^{3 / 2}}\left(1+O\left(s^{-1 / 4}\right)\right), \\
\text { as } s \rightarrow \infty, \quad \arg s \in\left(-\frac{\pi}{3}, \frac{\pi}{3}\right],
\end{array}
$$

or

$$
\begin{gathered}
u(s)=\frac{\alpha}{\sqrt{s}}+\sum_{n=1}^{[2 \alpha+1]} a_{n} s^{-\frac{3 n+1}{2}}+O\left(s^{-3[2 \alpha+1] / 2-1}\right) \\
+d_{-} s^{-3 \alpha+\frac{1}{2}} e^{-\frac{4}{3} s^{3 / 2}}\left(1+O\left(s^{-1 / 4}\right)\right), \\
\text { as } s \rightarrow \infty, \quad \arg s \in\left[-\frac{\pi}{3}, \frac{\pi}{3}\right),
\end{gathered}
$$


where

$$
d_{ \pm}=\frac{e^{ \pm 2 \alpha \pi i}-1}{\pi} 2^{-6 \alpha-\frac{5}{3}} \Gamma(1+2 \alpha) .
$$

Alternatively, the solution $u(s)$ can be characterized by the asymptotic relation

$$
\begin{aligned}
u(s)-u^{(\text {tri-tronq })}(s)=- & \frac{e^{-2 \alpha \pi i}-1}{\pi} 2^{-6 \alpha-\frac{5}{3}} \Gamma(1+2 \alpha) \\
& \quad \times s^{-3 \alpha+\frac{1}{2}} e^{-\frac{4}{3} s^{3 / 2}}\left(1+O\left(s^{-1 / 4}\right)\right), \quad \text { as } s \rightarrow+\infty .
\end{aligned}
$$

The Painlevé XXXIV tri-tronquée solution $u^{(t r i-t r o n q)}(s)$ is determined by the asymptotic condition

$$
u^{(\text {tri-tronq })}(s) \sim \frac{\alpha}{\sqrt{s}}+\sum_{n=1}^{\infty} a_{n} s^{-\frac{3 n+1}{2}}, \quad \text { as } s \rightarrow \infty, \quad \arg s \in\left(-\pi, \frac{\pi}{3}\right) .
$$

Finally, the coefficients $a_{n}$ of the asymptotic series above can be expressed in terms of the coefficients $b_{n}$ defined in (4.23), with $\nu$ replaced by $2 \alpha+1 / 2$ :

$$
2^{\frac{n+1}{2}} a_{n}=b_{n+1}-\frac{3 n-2}{2 \sqrt{2}} b_{n}+\frac{1}{2} \sum_{k, m \geq 1 ; k+m=n+1} b_{k} b_{m} .
$$

Remark 4.2 The leading asymptotics of the Painlevé II function $q(s)$ as $s \rightarrow+\infty$ is known (see [33]; see also [24, Chapter 10]). Unfortunately, the leading term is not enough to derive the corresponding asymptotics as $s \rightarrow-\infty$ of the Painlevé XXXIV function $u(s)$. Indeed, the leading asymptotics of $q(s)$ as $s \rightarrow+\infty$ is of the form

$$
q(s) \sim \sqrt{\frac{s}{2}} \cot \left(\frac{\sqrt{2}}{3} s^{3 / 2}+\chi\right),
$$

(the phase $\chi$ is known) and it cancels out in the right-hand side of equation (3.22). The better way to study the large negative $s$ asymptotics of the function $u(s)$ is via the direct analysis of the model $\mathrm{RH}$ problem for $\Psi_{\alpha}$. The case $\alpha=1$ shows that we might expect oscillating behavior as $s \rightarrow-\infty$ (see Figure [6) and indeed, assuming that $\alpha-1 / 2 \notin \mathbb{N}_{0}$, we are able to show that

$$
u(s)=\frac{\alpha}{\sqrt{-s}} \cos \left(\frac{4}{3}(-s)^{3 / 2}-\alpha \pi\right)+O\left(1 / s^{2}\right), \quad \text { as } s \rightarrow-\infty .
$$

The proof of (4.30) will be given in a future publication. Moreover, we conjecture that asymptotics (4.30) determines the solution $u(s)$ uniquely.

\section{Acknowledgements}

Alexander Its was supported in part by NSF grant \#DMS-0401009. Arno Kuijlaars is supported by FWO-Flanders project G.0455.04, by K.U. Leuven research grant OT/04/21, by the Belgian Interuniversity Attraction Pole P06/02, by the European Science Foundation Program MISGAM, and by a grant from the Ministry of Education and Science of Spain, project code MTM2005-08648-C02-01. Jörgen Östensson is supported by K.U. Leuven research grant OT/04/24. 


\section{References}

[1] M. Abramowitz and I. Stegun, Handbook of Mathematical Functions, Dover Publications, New York, 1992. Reprint of the 1972 edition.

[2] P. Bleher, A. Bolibruch, A. Its, and A. Kapaev, Linearization of the P34 equation of Painlevé-Gambier, unpublished manuscript.

[3] P. Bleher and A. Its, Semiclassical asymptotics of orthogonal polynomials, RiemannHilbert problem, and universality in the matrix model, Ann. Math. 150 (1999), 185266.

[4] P. Bleher and A. Its, Double scaling limit in the random matrix model: the RiemannHilbert approach, Comm. Pure Appl. Math. 56 (2003), 433-516.

[5] A. Borodin and P. Deift, Fredholm determinants, Jimbo-Miwa-Ueno $\tau$-functions, and representation theory, Comm. Pure Appl. Math. 55 (2002), 1160-1230.

[6] M.J. Bowick and E. Brézin, Universal scaling of the tail of the density of eigenvalues in random matrix models, Phys. Lett. B 268 (1991), 21-28.

[7] F. Carlson, Sur une classe de séries de Taylor, Dissertation, Uppsala, Sweden, 1914.

[8] T. Claeys and A.B.J. Kuijlaars, Universality of the double scaling limit in random matrix models, Comm. Pure Appl. Math. 59 (2006), 1573-1603.

[9] T. Claeys, A.B.J. Kuijlaars and M. Vanlessen, Multi-critical unitary random matrix ensembles and the general Painlevé II equation, to appear in Annals of Mathematics.

[10] T. Claeys and M. Vanlessen, Universality of a double scaling limit near singular edge points in random matrix models, arxiv: math-ph/0607043, to appear in Comm. Math. Phys.

[11] P. Deift, Orthogonal Polynomials and Random Matrices: A Riemann-Hilbert Approach, Courant Lecture Notes 3, New York University, 1999.

[12] P. Deift and D. Gioev, Universality at the edge of the spectrum for unitary, orthogonal and symplectic ensembles of random matrices, to appear in Comm. Pure Appl. Math.

[13] P. Deift, T. Kriecherbauer, and K.T-R McLaughlin, New results on the equilibrium measure for logarithmic potentials in the presence of an external field, J. Approx. Theory 95 (1998), 388-475.

[14] P. Deift, T. Kriecherbauer, K.T-R McLaughlin, S. Venakides, and X. Zhou, Uniform asymptotics for polynomials orthogonal with respect to varying exponential weights and applications to universality questions in random matrix theory, Comm. Pure Appl. Math. 52 (1999), 1335-1425.

[15] P. Deift, T. Kriecherbauer, K.T-R McLaughlin, S. Venakides, and X. Zhou, Strong asymptotics of orthogonal polynomials with respect to exponential weights, Comm. Pure Appl. Math. 52 (1999), 1491-1552. 
[16] P. Deift and X. Zhou, A steepest descent method for oscillatory Riemann-Hilbert problems. Asymptotics for the MKdV equation, Ann. Math. 137 (1993), 295-368.

[17] P. Deift and X. Zhou, Long-time asymptotics for solutions of the NLS equation with initial data in a weighted Sobolev space, Comm. Pure Appl. Math. 56 (2003), 10291077.

[18] P. Deift and X. Zhou, Perturbation theory for infinite-dimensional integrable systems on line. A case study, Acta Math. 188 (2002), 163-262.

[19] P. Deift and X. Zhou, A priori $L^{p}$-estimates for solutions of Riemann-Hilbert problems, Int. Math. Research Notices 2002 (2002), 2121-2154.

[20] M. Duits and A.B.J. Kuijlaars, Painlevé I asymptotics for orthogonal polynomials with respect to a varying quadratic weight, Nonlinearity 19 (2006), 2211-2245.

[21] F.J. Dyson, Correlation between the eigenvalues of a random matrix, Comm. Math. Phys. 19 (1970), 235-250.

[22] H. Flaschka and A.C. Newell, Monodromy and spectrum-preserving deformations I, Comm. Math. Phys. 76 (1980), 65-116.

[23] A.S. Fokas, A.R. Its, and A.V. Kitaev, The isomonodromy approach to matrix models in 2D quantum gravity, Comm. Math. Phys. 147 (1992), 395-430.

[24] A.S. Fokas, A.R. Its, A.A. Kapaev and V.Yu. Novokshenov, Painlevé Transcendents, the Riemann-Hilbert approach, Math. Surveys and Monogr. 128, Amer. Math. Soc., Providence RI, 2006

[25] A.S. Fokas and X. Zhou, On the solvability of Painlevé II and IV, Commun. Math. Phys. 144 (1992), 601-622.

[26] P.J. Forrester, The spectrum edge of random matrix ensembles, Nucl. Phys. B 402 (1993), 709-728.

[27] J. Harnad and A.R. Its, Integrable Fredholm operators and dual isomonodromic deformations, Comm. Math. Phys. 226 (2002), 497-530.

[28] S.P. Hastings and J.B. McLeod, A boundary value problem associated with the second Painlevé transcendent and the Korteweg-de Vries equation, Arch. Rational Mech. Anal. 73 (1980), 31-51.

[29] E.L. Ince, Ordinary Differential Equations, Dover, New York, 1956.

[30] A.R. Its and A.A. Kapaev, The irreducibility of the second Painlevé equation and the isomonodromy method. In: Toward the exact WKB analysis of differential equations, linear or non-linear, C.J. Howls, T. Kawai, and Y. Takei, eds., Kyoto Univ. Press, 2000, pp. 209-222.

[31] M. Jimbo, T. Miwa, and K. Ueno, Monodromy preserving deformation of linear ordinary differential equations with rational coefficients, Physica D 2 (1981), 306352. 
[32] S. Kamvissis, K.D.T-R McLaughlin, and P.D. Miller, Semiclassical Soliton Ensembles for the focusing Nonlinear Schrödinger Equation, Ann. Math. Studies 154, Princeton Univ. Press, Princeton, 2003.

[33] A.A. Kapaev, Global asymptotics of the second Painlevé transcendent, Phys. Lett. A, 167 (1992) 356-362.

[34] A.A. Kapaev, Quasi-linear Stokes phenomenon for the Hastings-McLeod solution of the second Painlevé equation, arXiv: nlin.SI/0410009

[35] A.A. Kapaev and E. Hubert, A note on the Lax pairs for Painlevé equations, J. Phys. A: Math. Gen. 32 (1999), 8145-8156.

[36] A.B.J. Kuijlaars and K.T-R McLaughlin, Generic behavior of the density of states in random matrix theory and equilibrium problems in the presence of real analytic external fields, Comm. Pure Appl. Math. 53 (2000), 736-785.

[37] A.B.J. Kuijlaars and M. Vanlessen, Universality for eigenvalue correlations at the origin of the spectrum, Comm. Math. Phys. 243 (2003), 163-191.

[38] M.L. Mehta, Random Matrices, 2nd. ed. Academic Press, Boston, 1991.

[39] G. Moore, Matrix models of 2D gravity and isomonodromic deformations, Progr. Theor. Phys. Suppl. 102 (1990), 255-285.

[40] E.B. Saff and V. Totik, Logarithmic Potentials with External Fields, Springer-Verlag, New-York, 1997.

[41] M. Reed and B. Simon, Methods of Modern Mathematical Physics IV, Academic Press, New York-London, 1978.

[42] C. Tracy and H. Widom, Level spacing distributions and the Airy kernel, Comm. Math. Phys. 159 (1994), 151-174.

[43] C. Tracy and H. Widom, Airy kernel and Painlevé II. In: Isomonodromic Deformations and Applications in Physics, (J. Harnad and A. Its, eds), CRM Proc. Lecture Notes, 31, Amer. Math. Soc., Providence, RI, 2002. pp. 85-96.

[44] X. Zhou, The Riemann-Hilbert problem and inverse scattering, SIAM J. Math. Anal. 20 (1989), 966-986. 